University of Louisville

ThinkIR: The University of Louisville's Institutional Repository

$12-2006$

\title{
Mobility-based predictive call admission control and resource reservation for next-generation mobile communications networks.
}

Sherif Said Rashad 1974-

University of Louisville

Follow this and additional works at: https://ir.library.louisville.edu/etd

\section{Recommended Citation}

Rashad, Sherif Said 1974-, "Mobility-based predictive call admission control and resource reservation for next-generation mobile communications networks." (2006). Electronic Theses and Dissertations. Paper 1182.

https://doi.org/10.18297/etd/1182

This Doctoral Dissertation is brought to you for free and open access by ThinkIR: The University of Louisville's Institutional Repository. It has been accepted for inclusion in Electronic Theses and Dissertations by an authorized administrator of ThinkIR: The University of Louisville's Institutional Repository. This title appears here courtesy of the author, who has retained all other copyrights. For more information, please contact thinkir@louisville.edu. 


\title{
MOBILITY-BASED PREDICTIVE CALL ADMISSION \\ CONTROL AND RESOURCE RESERVATION FOR \\ NEXT-GENERATION MOBILE COMMUNICATIONS NETWORKS
}

By

Sherif Said Rashad

B.Sc. in Electrical Engineering, Zagazig University, Egypt, 1996

M.Sc. in Electrical Engineering, Zagazig University, Egypt, 2001

\author{
A Dissertation \\ Submitted to the Faculty of the \\ Graduate School of the University of Louisville \\ in Partial Fulfillment of the Requirements \\ for the Degree of
}

Doctoral of Philosophy

Computer Engineering and Computer Science Department

Speed School of Engineering

University of Louisville

Louisville, Kentucky

December 2006 


\title{
MOBILITY-BASED PREDICTIVE CALL ADMISSION \\ CONTROL AND RESOURCE RESERVATION FOR \\ NEXT-GENERATION MOBILE COMMUNICATIONS NETWORKS
}

\author{
By \\ Sherif Said Rashad \\ M.Sc. in Electrical Engineering, Zagazig University, Egypt, 2001 \\ B.Sc. in Electrical Engineering, Zagazig University, Egypt, 1996
}

A Dissertation Approved on

October 18, 2006

By the Following Reading and Examination Committee:

Dr. Mehmed Kantardzic (Dissertation Director)

Dr. Anup Kumar (Dissertation Co-Director)

Dr. Adel Elmaghraby

Dr. Olfa Nasraoui

Dr. John Usher 


\section{ACKNOWLEDGMENT}

First and foremost, I am very thankful to God for every thing, without his blessing this research would not have been possible. I would like to express my deepest thanks and appreciation to my dissertation advisors: Dr. Mehmed Kantardzic and Dr. Anup Kumar for their outstanding guidance, active support, cooperation, and encouragement to bring this work to success. My deepest thanks are extended also to Dr. Adel Elmaghraby for serving in my dissertation committee and for his encouragement and support to complete this research. I would like also to thank Dr. Olfa Nasraoui and Dr. John Usher for serving in my dissertation committee and for their encouragement and suggestions.

I would like also to thank my mother and my father for their prayers, encouragement, and support during my study. My special thanks should be extended also to my great wife, Heba, for her love, patience, support, and encouragement during this research. I would like also to thank my sons, Mohammad and Khalid who are really the joy of my life. At the end, I would like to thank the staff in the Computer Engineering and Computer Science Department at the University of Louisville, my colleagues in the Data Mining Laboratory, and my colleagues in the Mobile Information Networks and Distributed Systems (MINDS) Laboratory for their help and support during this research. 


\title{
ABSTRACT
}

\section{MOBILITY-BASED PREDICTIVE CALL ADMISSION CONTROL AND RESOURCE RESERVATION FOR NEXT-GENERATION MOBILE COMMUNICATIONS NETWORKS}

\author{
Sherif Said Rashad
}

October 18,2006

Recently, the need for wireless and mobile communications has grown tremendously and it is expected that the number of users to be supported will increase with high rates in the next few years. Not only the number of users, but also the required bandwidth to support each user is supposed to increase especially with the deploying of the multimedia and the real time applications. This makes the researchers in the filed of mobile and wireless communications more interested in finding efficient solutions to solve the limitations of the available natural radio resources.

One of the important things to be considered in the wireless mobile environment is that the user can move from one location to another when there is an ingoing call. Resource reservation $(R R)$ schemes are used to reserve the bandwidth $(B W)$ required for the handoff calls. This will enable the user to continue his /her call while he/she is moving. Also, call admission control $(C A C)$ schemes are used as a provisioning strategy to limit the number of call connections into the network in order 
to reduce the network congestion and the call dropping. The problem of CAC and RR is one of the most challenging problems in the wireless mobile networks.

Also, in the fourth generation $(4 G)$ of mobile communication networks, many types of different mobile systems such as wireless local area networks (WLANs) and cellular networks will be integrated. The $4 \mathrm{G}$ mobile networks will support a broad range of multimedia services with high quality of service. New Call demission control and resource reservation techniques are needed o support the new $4 \mathrm{G}$ systems.

Our research aims to solve the problems of Call Admission Control (CAC), and resource reservation (RR) in next-generation cellular networks and in the fourth generation $(4 \mathrm{G})$ wireless heterogeneous networks.

In this dissertation, the problem of CAC and RR in wireless mobile networks is addressed in detail for two different architectures of mobile networks: 1) cellular networks, and 2) wireless heterogeneous networks (WHNs) which integrate cellular networks and wireless local area networks (WLANs). We have designed, implemented, and evaluated new mobility-based predictive call admission control and resource reservation techniques for the next-generation cellular networks and for the $4 \mathrm{G}$ wireless heterogeneous networks. These techniques are based on generating the mobility models of the mobile users using one-dimensional and multidimensional sequence mining techniques that have been designed for the wireless mobile environment. The main goal of our techniques is to reduce the call dropping probability and the call blocking probability, and to maximize the bandwidth utilization $\mathrm{n}$ the mobile networks. By analyzing the previous movements of the mobile users, we generate local and global mobility profiles for the mobile users, 
which are utilized effectively in prediction of the future path of the mobile user. Extensive simulation was used to analyze and study the performance of these techniques and to compare its performance with other techniques. Simulation results show that the proposed techniques have a significantly enhanced performance which is comparable to the benchmark techniques. 


\section{TABLE OF CONTENTS}

ACKNOWLEDGMENT iii

ABSTRACT iv

LIST OF FIGURES. $\mathbf{x i}$

LIST OF TABLES xv

\section{CHAPTER}

1. INTRODUCTION 1

1.1. OVERVIEW OF THE CAC AND RR .................................................. 2

1.2. GoAls ANd OBJECTIVES OF OUR RESEARCH ........................................ 4

1.3. ORGANIZATION OF THE DiSSERTATION ................................................ 5

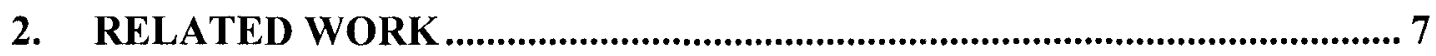

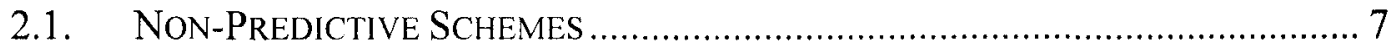

2.2. Predictive SCHEMES .................................................................... 9

2.2.1. Prediction Using Current Mobility Factors .................................... 9

2.2.2. Prediction Using Previous Movements' History ............................. 12

2.3. LIMITATIONS OF EXISTING CAC AND RR TECHNIQUES:.......................... 16

3. PREDICTIVE CAC AND RR FOR NEXT-GENERATION CELLULAR

NETWORKS: PCAC-RR 18 


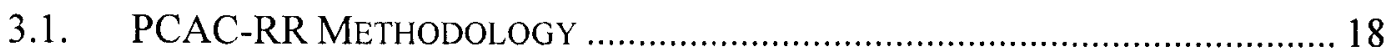

3.2. ARChitecture of Cellular Networks Supported by PCAC-RR........ 21

3.3. InduCtive LEaRning through Mobile Data Mining .............................. 23

3.3.1. Generation of Local Mobility Profiles ................................................ 24

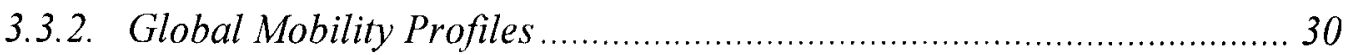

3.4. Predictive Resource Reservation SChEme (RR) .................................. 32

3.5. Predictive Call Admission Control Scheme (PCAC)........................... 34

\section{MULTIDIMENSIONAL SEQUENTIAL MINING FOR MOBILE}

NETWORKS: MOBILEPREFIXSPAN ............................................................. 36

4.1. InPUT AND OUTPUTS IN MOBILEPREFIXSPan: .................................................. 36

4.2. Problem Definition of Sequence Mining in the Mobile Network

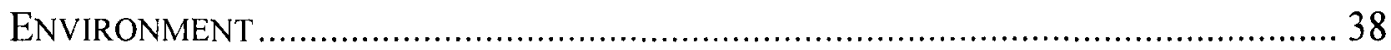

4.3. MobILEPREFIXSPAN ALGORITHM .......................................................... 42

4.4. Calculating the Support of the Generated Sequential Patterns. 48

5. MSP-CACRR: MULTIDIMENSIONAL SEQUENTIAL PATTERNS

BASED ADMISSION CONTROL FOR NEXT-GENERATION CELLULAR

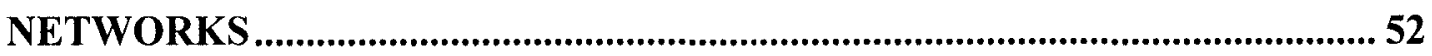

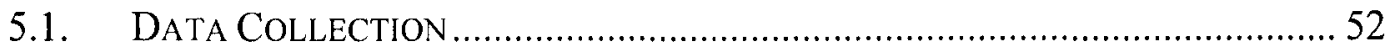

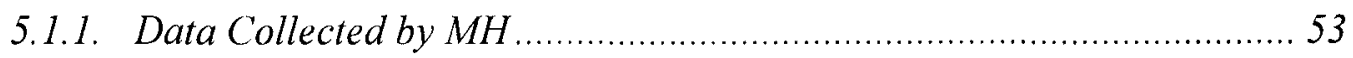

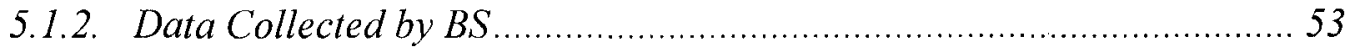

5.2. Predictive Resource ReSERVATION SCHEME ......................................... 56

5.3. Predictive Call Admission Control Scheme........................................ 58 


\section{PAC-WHN: PREDICTIVE ADMISSION CONTROL FOR WIRELESS HETEROGENEOUS NETWORKS}

6.1. INTRODUCTION 63

6.2. ARChiteCture of 4G Mobile Communication Systems Supported By PAC-WHN 64

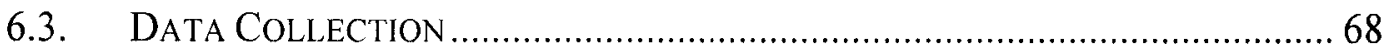

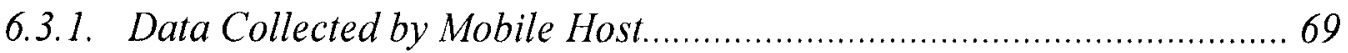

6.3.2. Data Collected by Base Station and Access Point ............................... 71

6.4. RESOURCE RESERVATION IN PCA-WHN ................................................. 73

6.5. ADMISSION CONTROL IN PCA-WHN ………….................................... 78

7. SIMULATION AND EXPERIMENTAL RESULTS...................................... 81

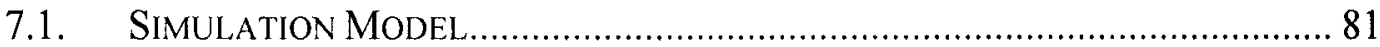

7.2. EXPERIMENTAL RESULTS FOR PCAC-RR …………................................. 84

7.2.1. Performance of PCAC-RR Using Different Types of Mobility Profiles. 84

7.2.2. Performance of PCAC-RR Using Different Number of Day Time

Intervals 86

7.2.3. Study of the Effect of Predicting the Handoff Time Using the Repeated Sequences. 90

7.2.4. Comparison between the PCAC-RR Technique and Other Techniques. 93

7.3. EXPERIMENTAL RESULTS FOR MSP-CACRR 98

7.3.1. Study of the Effect of Using MobilePrefixSpan for Mining the Sequential Patterns 98 
7.3.2. Study the Effect of Using the Type of Service as One of the Dimensions 102

7.3.3. Study of the Effect of Using the Type of the Day as One of the Dimensions. 104

7.3.4. Performance of MSP-CACRR Technique Compared to Other Techniques 107

7.4. EXPERIMENTAL RESULTS FOR PCA-WHN ……....................................... 110

7.4.1. Study of the Performance of PCA-WHN Technique ........................... 110

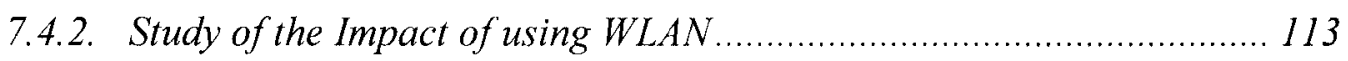

8. CONCLUSION AND FUTURE WORK ....................................................116

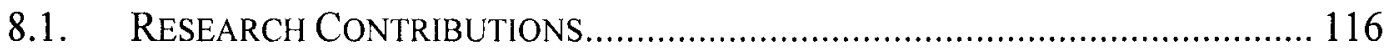

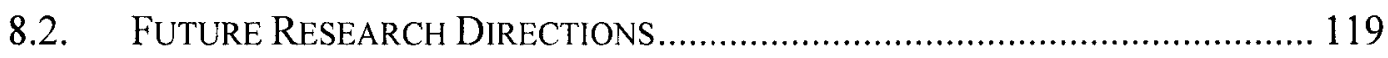

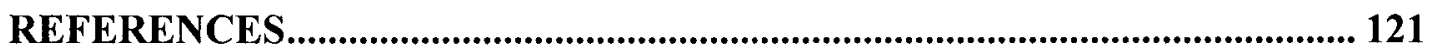

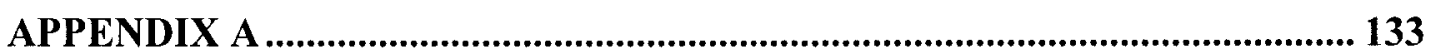

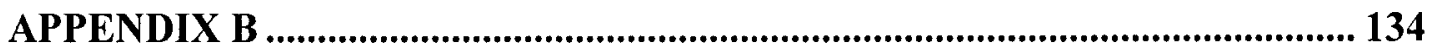

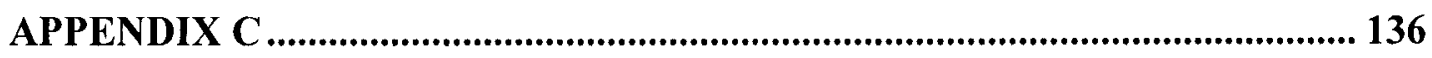

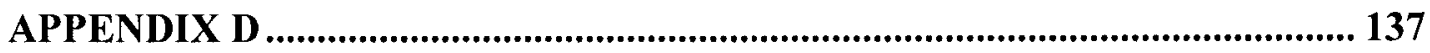

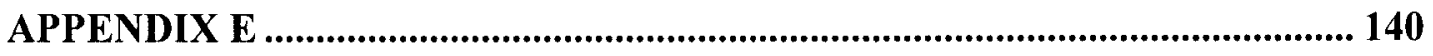

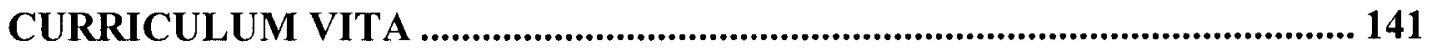




\section{LIST OF FIGURES}

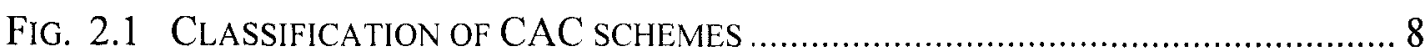

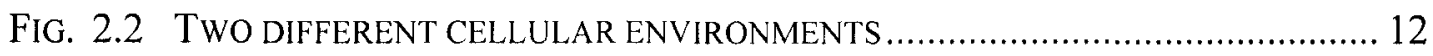

FIG. 2.3 AN EXAMPLE OF THE MOBILITY GRAPH PROPOSED IN [3] …....................... 14

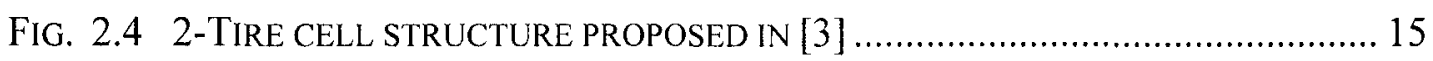

FIG. 3.1 THE SYSTEM ARCHITECTURE SUPPORTED BY THE PCAC-RR TECHNIQUE.... 20

Fig. 3.2 Cellular Networks ARChitecture …..................................................... 22

Fig. 3.3 Path generation PROCESS For Local Paths .......................................... 26

FIG. 4.1 INPUTS AND OUTPUTS FOR MOBILEPREFIXSPAN TECHNIQUE ....................... 37

Fig. 6.1 Architecture of Proposed Wireless Heterogeneous Networks..... 66

Fig. 6.2 Distribution OF THE TOTAL RESOURCES IN BS/AP AND HOW TO USE THESE

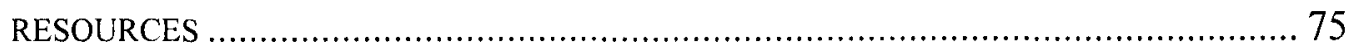

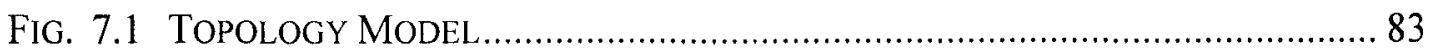

Fig. 7.2 CAll Blocking PROBABILITY RESUlts COMPaRING BETWEEN THE MOBILITY

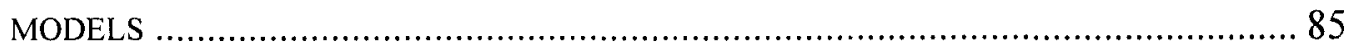

Fig. 7.3 CALL DROPPING PROBABILITY RESULTS COMPARING BETWEEN THE MOBILITY

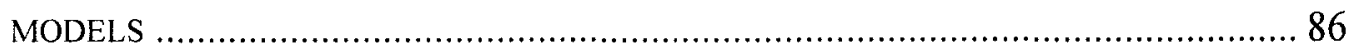

FIG. 7.4 BANDWIDTH UTILIZATION RESULTS COMPARING BETWEEN THE MOBILITY MODELS 86 
Fig. 7.5 STUDY THE EFFeCt OF THE NUMBER OF TIME INTERVALs $\left(\mathrm{N}_{\mathrm{T}}\right)$ ON THE CDP FOR PCAC-RR TECHNIQUE.

Fig. 7.6 Study THE EFFECT OF THE NUMBER OF TIME INTERVALS $\left(\mathrm{N}_{\mathrm{T}}\right)$ ON THE CDP FOR PCAC-RR TECHNIQUE.

Fig. 7.7 StUdy THE EFFECT OF THE NUMBER OF TIME INTERVALS $\left(\mathrm{N}_{\mathrm{T}}\right)$ ON THE BWU FOR PCAC-RR TECHNIQUE. 90

Fig. 7.8 StUdy of THE EFFECT OF USING THE REPEATED SEQUENCES ON THE CBP FOR PCAC-RR TECHNIQUE .92

Fig. 7.9 STUDY OF THE EFFECT OF USING THE REPEATED SEQUENCES ON THE CDP FOR PCAC-RR TECHNIQUE. 92

Fig. 7.10 STUdy OF THE EFFECT OF USING THE REPEATED SEQUENCES ON THE BWU FOR PCAC-RR TECHNIQUE. 93

FIG. 7.11 CALL BLOCKING PROBABILITY RESULTS COMPARING BETWEEN DIFFERENT SCHEMES 95

Fig. 7.12 Percentage of IMPROVEMENT IN THE CBP FOR PCAC-RR COMPARING TO PR-CAT4 .95

FIG. 7.13 CALL DROPPING PROBABILITY RESULTS COMPARING BETWEEN DIFFERENT SCHEMES .96

Fig. 7.14 Percentage of IMPRovement IN THE CDP FOR PCAC-RR COMPARING TO PR-CAT4 96

FIG. 7.15 BANDWIDTH UTILIZATION RESULTS COMPARING BETWEEN DIFFERENT SCHEMES .97 
Fig. 7.16 PeRCENTAge Of IMPROVEMENT IN THE CDP FoR PCAC-RR COMPARING TO PR-CAT4 97

Fig. 7.17 CALL BLOCKING PROBABILITY RESULTS COMPARING BETWEEN MSP$\operatorname{CACRR}\left(D_{1}\right)$ AND PCACRR 100

FIG. 7.18 CALL DROPPING PROBABILITY RESULTS COMPARING BETWEEN MSP$\operatorname{CACRR}\left(\mathrm{D}_{1}\right)$ AND PCACRR 101

Fig. 7.19 BANDWIDTh UTILIZATION RESULTS COMPARING BETWEEN MSP-

$\operatorname{CACRR}\left(\mathrm{D}_{1}\right)$ AND PCACRR 101

Fig. 7.20 CALL BLOCKING PROBABILITY RESULTS COMPARING BETWEEN MSP$\operatorname{CACRR}\left(\mathrm{D}_{1}\right)$ AND MSP-CACRR $\left(\mathrm{D}_{1}, \mathrm{D}_{3}\right)$ 103

FIG. 7.21 CALL DROPPING PROBABILITY RESULTS COMPARING BETWEEN MSP-

$\operatorname{CACRR}\left(\mathrm{D}_{1}\right)$ AND MSP-CACRR $\left(\mathrm{D}_{1}, \mathrm{D}_{3}\right)$ 103

FIG. 7.22 BANDWIDTH UTILIZATION RESULTS COMPARING BETWEEN MSP-

$\operatorname{CACRR}\left(\mathrm{D}_{1}\right)$ AND MSP-CACRR $\left(\mathrm{D}_{1}, \mathrm{D}_{3}\right)$ 104

Fig. 7.23 CALL BLOCKING PROBABILITY RESULTS COMPARING BETWEEN MSP-

$\operatorname{CACRR}\left(D_{1}\right)$ AND MSP-CACRR $\left(D_{1}, D_{2}\right)$ 105

FIG. 7.24 CALL DROPPING PROBABILITY RESULTS COMPARING BETWEEN MSP-

$\operatorname{CACRR}\left(D_{1}\right)$ AND MSP-CACRR $\left(D_{1}, D_{2}\right)$ 106

FIG. 7.25 BANDWIDTH UTILIZATION RESULTS COMPARING BETWEEN MSP$\operatorname{CACRR}\left(D_{1}\right)$ AND MSP-CACRR $\left(D_{1}, D_{2}\right)$ 106

Fig. 7.26 CALL BLOCKING PROBABILITY RESULTS COMPARING BETWEEN MSP$\operatorname{CACRR}\left(\mathrm{D}_{1}, \mathrm{D}_{2}\right)$ AND OTHER TECHNIQUES 108 
Fig. 7.27 CALL DROPPING PROBABILITY RESULTS COMPARING BETWEEN MSP-

$\operatorname{CACRR}\left(D_{1}, D_{2}\right)$ AND OTHER TECHNIQUES 109

FIG. 7.28 BANDWIDTH UTILIZATION RESULTS COMPARING BETWEEN MSP-

$\operatorname{CACRR}\left(\mathrm{D}_{1}, \mathrm{D}_{2}\right)$ AND OTHER TECHNIQUES 109

FIG. 7.29 CALL BLOCKING PROBABILITY RESULTS COMPARING BETWEEN PCA-WHN AND BENCHMARK TECHNIQUE 111

Fig. 7.30 CALL BLOCKING PROBABILITY RESULTS COMPARING BETWEEN PCA-WHN AND BENCHMARK TECHNIQUE

Fig. 7.31 BANDWIDTH UTILIZATION RESULTS COMPARING BETWEEN PCA-WHN AND BENCHMARK TECHNIQUE

FIG. 7.32 CALL BLOCKING PROBABILITY RESULTS COMPARING BETWEEN CELLULAR AND HETEROGENEOUS NETWORKS 114

Fig. 7.33 CALL DROPPING PROBABILITY RESULTS COMPARING BETWEEN CELLULAR AND HETEROGENEOUS NETWORKS 114

FIG. 7.34 BANDWIDTH UTILIZATION RESULTS COMPARING BETWEEN CELLULAR AND HETEROGENEOUS NETWORKS 115 


\section{LIST OF TABLES}

TABLE 3.1 EXAMPLE OF THE COLLECTED DATA IN THE MH ……................................ 25

TABLE 3.2 EXAMPLE OF THE DISCOVERED LOCAL PATHS FOR A SINGLE USER …….. 28

TABLE 3.3 EXAMPLE OF THE LOCAL PROFILES FOR A SINGLE USER COVERING DIFFERENT TIME INTERVALS $T_{t}$ 30

TABLE 4.1 MULTIDIMENSIONAL SEQUENCES DATASET COLLECTED By MOBILE USER 46

TABle 4.2 Conversion of TABle 3 to One-Dimension SequenCes dataseT ......... 46

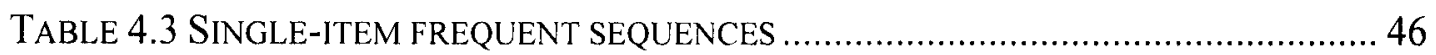

Table 4.4 Complete Sequential Patterns for the given DataSET .................... 47

TABLE 4.5 STORED SEQUENTIAL PATTERNS AND CORRESPONDING SUPPORT AND

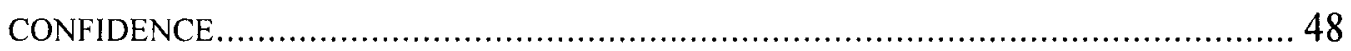

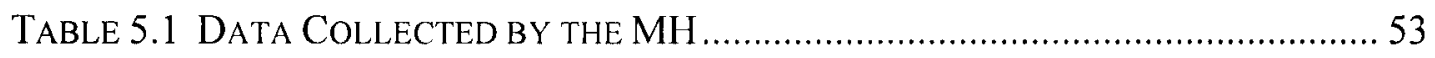

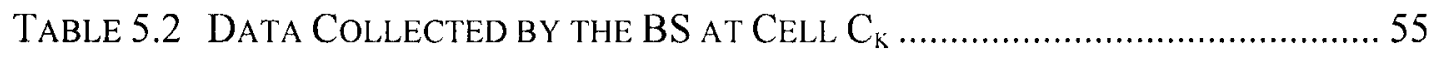

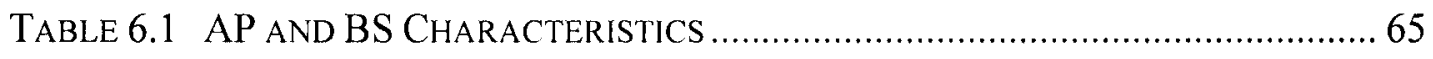

Table 6.2 Traffic Class for 4G Wireless Heterogeneous Networks [60] .... 67

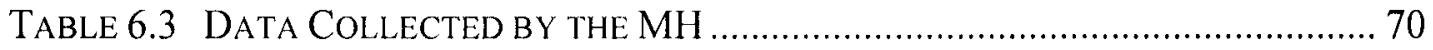

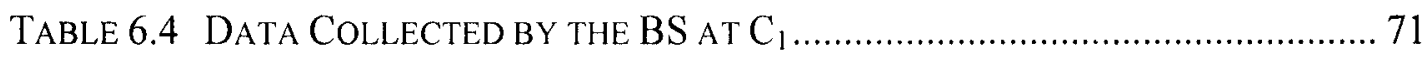

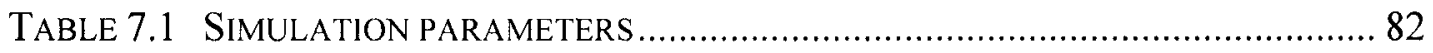

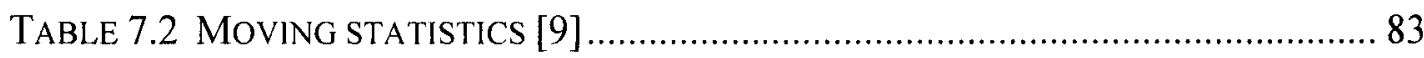

TABLE 7.3 THE TIME INTERVALS $T_{I}$ USED IN THE EXPERIMENTS................................ 88 
TABle 7.4 Updated Simulation Parameters. 


\section{CHAPTER I}

\section{INTRODUCTION}

The rapid advance in wireless and mobile communications field is providing many new and improved services to their customers. While the first generation of mobile networks supported only analog services, the second generation, which is currently deployed, can provide many digital services such as digital voice and lowrate circuit-switched data services. The third-generation $(3 G)$ systems were aimed at providing multimedia mobile services and achieving a maximum bit rate of $2 \mathrm{Mb} / \mathrm{s}$ [1]. The migration of $3 \mathrm{G}$ networks has already begun in different regions, and researchers are thinking how $3 G$ networks will evolve to fourth-generation $(4 G)$ systems [1] where the mobile technologies will be integrated to provide the required services. In these new generations of mobile communications networks (third generation (3G), beyond third generation $(B 3 G)$, and fourth generation $(4 \mathrm{G}))$, it is required that networks support a broad range of multimedia services which includes real time applications with a required level of quality of service $(Q o S)$ [2]. The big dream in the communication industry is to have the wireless mobile services any time, any where and for all types of applications [3]. It is expected that in the future we will reach the point where the number of worldwide wireless subscribers will be 
higher than the number of wireline subscribers [4]. One of the basic limitations of the wireless mobile networks is the scarcity of the available bandwidth.

In the first section of this chapter, we give a general overview of the Call Admission Control $(C A C)$ and Resource Reservation $(R R)$ problems. The goals and the objectives of this research are given in the second section. The organization of the dissertation is given in the third section.

\subsection{Overview of the CAC and RR}

There are two basic characteristics of the wireless mobile networks that are important to manage the available radio resources: mobility of the users and the limited communications bandwidth. Mobility provides the possibility to communicate in different locations and while on-the- move [5]. The user is supposed to have the service at any location covered by the serving network. The totally free mobility is the ultimate goal from the user respective. From the service provider perspective, the mobility of the users can cause a significant overhead in the management of available bandwidth if the nature of this mobility has not been considered in the design of the bandwidth reservation schemes. Because of the mobility of the users, it is required in the mobile communication environment to reserve the bandwidth for the served users in the neighbor cells to ensure the continuity of the calls without interruption while the user is moving form one location to another.

Two main reasons affect the available bandwidths: the increasing number of mobile users, and the high bandwidth required by the new multimedia services (video, images, audio, data ...etc.). Therefore the required resources (bandwidths) to be reserved are increasing tremendously for the next generation of mobile networks. 
The mobile network has to control the admission of new admitted calls (this is known as the call admission control problem), and also it has to manage existing calls to reserve efficiently the required bandwidth (this is known as the resource reservation problem) to provide the service without interruption and for a large number of customers. This makes the problem of call admission control $(C A C)$ and Resource Reservation $(R R)$ one of the most challenging problems in the next generation of mobile networks. In this dissertation, we are introducing a new solution to solve the problem of CAC and RR.

Call (or connection) admission control refers to the task of deciding if a new call should be admitted into and supported by the network [6]. CAC is considered as a provisioning strategy to limit the number of call connections into the network in order to reduce the network congestion and the call dropping [7]. The mobility of the users makes the dropping of the call more possible in the mobile networks. Also, the new call may not be accepted if there is not enough bandwidth to support this call. To ensure a given QoS, the service providers have to maintain the ongoing calls when the mobile users move from one cell to another cell and it also has to accept new calls. The handoff occurring form existing calls will always have a priority over the new arriving calls. Because of user's mobility, resource reservation (RR) is used to allocate the bandwidth required for the handoff calls. This reservation is performed in the cells that will be visited by the user during his/her movement and will facilitate the completion of the ongoing call without dropping.

The problems of call admission control and resource reservation are the main topic of this dissertation and will be explained in details in this chapter and in the 
remaining chapters of this dissertation. We studied this problem for two different structures of mobile networks: 1) cellular mobile networks, and 2) wireless heterogeneous networks.

When there is not enough bandwidth to serve the handoff calls, this call may be dropped. In addition, if there is not enough bandwidth to serve the new arriving call, these calls may be blocked (rejected). Good CAC and RR schemes have to make a balance between the call blocking and call dropping in order to provide the required QoS [8-12]. Bandwidth utilization is also an important parameter used to measure the efficiency of using the reserved bandwidth. Bandwidth utilization expresses the ratio between the amount of bandwidth used by various applications admitted into the network, and either the total requested bandwidth or the total available bandwidth, whichever is smaller $[6,13,14]$.

Call blocking probability (CBP), call dropping probability (CDP), and bandwidth utilization $(B W U)$ are the three important metrics for the quality of service (QoS) which will be considered in this dissertation. Keeping the call dropping probability and the call blocking probability as low as possible while maximizing the bandwidth utilization is one of the greatest challenges in mobile networks $[15,16]$.

\subsection{Goals and Objectives of Our Research}

The basic goal of our research is to improve the performance of the wireless mobile communication networks. The basic problems in wireless mobile networks that we are trying to solve are call admission control (CAC) and resource reservation (RR) problems. The main objectives of the CAC and RR are:

1. Reducing the call dropping probability (for handoff calls) 
2. Reducing the call blocking probability (for new calls)

3. Reducing the network congestion

4. Maximizing the utilization of the bandwidth (which is the main resource in wireless systems) as long as the required QoSs for all calls are guaranteed.

The main objectives of this research are to solve the problems of CAC and RR in two different structures of wireless networks:

1. Cellular Mobile Networks:

In this structure we have only one type of the radio access networks (RAN). The mobile services are only supported by the cellular networks which can provide all types of services including the multimedia applications and data communications.

\section{Wireless Heterogeneous Networks:}

This structure represents the main structure in the fourth generation (4G) of mobile networks. It integrates different kind of RANs such as the cellular networks and the wireless local area networks (WLANs). This will help to provide better services with high rates and to accommodate more users. But there is a need for CAC and RR techniques that take into account the advantages of this integrated structure and help to enhance the overall performance.

\subsection{Organization of the Dissertation}

The remainder of this dissertation is organized as follows. Chapter 2 gives an overview of call admission control and resource reservation schemes that have been 
proposed in the literature. Chapter 3 introduces the proposed predictive based CAC and RR technique (PCAC-RR) for cellular networks. The processes of building the local and global mobility profiles are explained in this section as well as the RR and the CAC schemes. Chapter 4 explains the details of the data mining technique, called MobilePrefixSpan, which we have developed to analyze the information collected from the mobile users and extract the mobility patterns. In Chapter 5, a multidimensional sequential patterns (MSP) based CAC and RR technique called MSP-CACRR technique is explained in detail to solve the CAC and RR problems in the next generation of cellular networks. Chapter 6 explains a new predictive CAC and RR technique that can support the $4 \mathrm{G}$ wireless heterogeneous networks (WHN). This new technique is based on an efficient multidimensional sequence mining technique MobilePrefixSpan. Chapter 7 describes the simulation environment with its parameters, and all of the experimental results for the simulations that have been conducted to evaluate the performance of the proposed CAC and RR techniques. Finally, we give our conclusions and future research directions in Chapter 8 . 


\section{CHAPTER II}

\section{RELATED WORK}

According to the nature of the previous solutions that have been introduced in the literature to solve problem of $\mathrm{CAC}$, we suggest to classify the CAC schemes into two main categories depending on the prediction of the user's movements: a) predictive schemes and b) non-predictive schemes, as shown in Fig. 2.1. It has been proven that predictive techniques that use the mobility information of the users to predict the next cell are more effective than the non predictive techniques.

\subsection{Non-Predictive Schemes}

Many non-predictive $\mathrm{CAC}$ schemes for mobile communications have been developed. In [7], a number of classical schemes for CAC were reintroduced taking into account more general assumptions such as inequality of the average values of channel holding times for new calls and handoff calls. Two types of non-predictive schemes are presented in that paper, these include the guard channel schemes and the queering property schemes. In Guard Channel (GC) scheme, some channels are reserved for handoff calls [7]. There are four different schemes: the cutoff priority scheme (reserve a portion of channel for handoff calls), the fractional guard channel schemes (admit new call with certain probability), the rigid division-based CAC 
scheme (divide all channels allocated to a cell into two groups: one for the common use for all calls and the other for handoff calls only), and new call bounding scheme (limit the number of new calls admitted to the network). In Queuing Priority (QP) schemes, calls are accepted whenever there are free channels [7]. When all channels are busy, new calls are blocked while handoff calls are queued, or all arriving calls are queued with certain rearrangements in the queue [7].

The non-predictive algorithms don't consider the mobility information about the users. Therefore, these kinds of approaches are not suitable for mobile environment because the mobility of the user in wireless networks is one of the most important features. Prediction of movements has been used to enhance the performance of the resource reservation, location management and handover management in mobile networks [17-25].

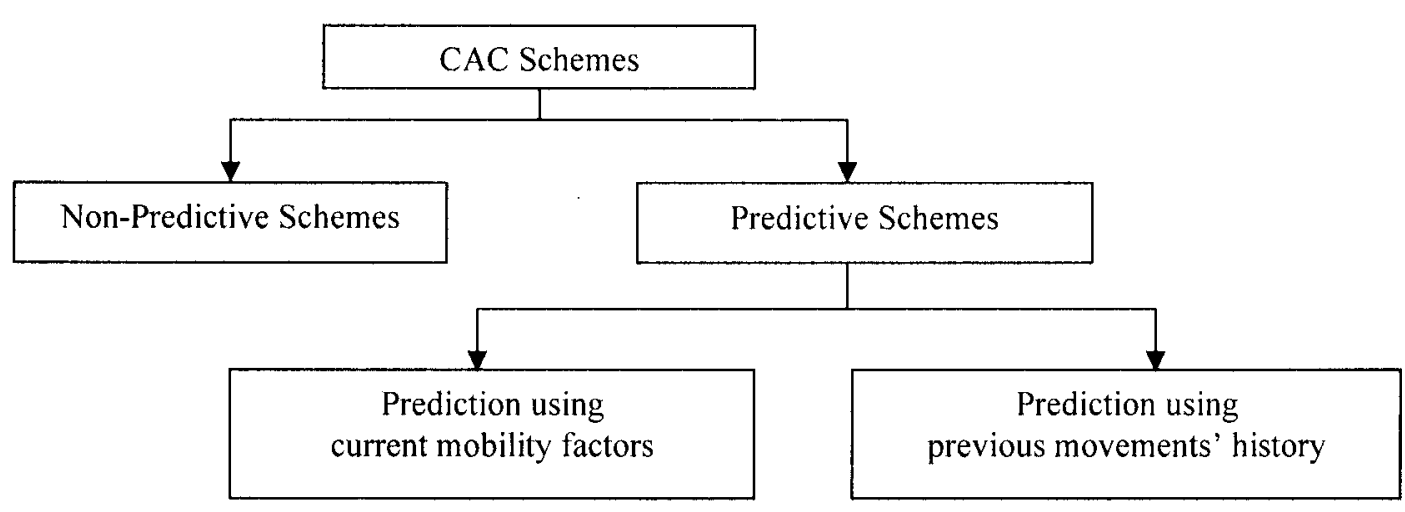

Fig. 2.1 Classification of CAC schemes 


\subsection{Predictive Schemes}

Recently, several articles [7, 21-24, 26-34] describe mobility-based predictive schemes for CAC and RR to enhance the performance of mobile networks in terms of QoS metrics. These predictive techniques can be classified into two main subcategories (as shown in Figure 2). The first subcategory includes the schemes that use the current mobility factors (velocity, direction, angle, or distance) to estimate the next cell. The movement history of the user is not used in these schemes. The second subcategory includes these schemes that are based on the movements' history of the user over a suitable period of time to predict the next base station. The technique we are introducing in this dissertation (PCAC-RR) falls in the second subcategory of predictive CAC schemes.

\subsubsection{Prediction Using Current Mobility Factors}

The suggested approach in [26] uses three mobility parameters (velocity, distance, direction) for resource reservation. Also, an on-demand bandwidthborrowing scheme is introduced to guarantee the QoS requirements. This approach is mainly developed to solve the problem occurs when the mobile user changes his/her direction and speed suddenly especially at the border of the cells [26]. In these situations, the bandwidth will be reserved in a wrong cell. The authors solve this problem by borrowing bandwidth from rate adaptive calls in the new cell entered by the mobile terminal (MT). In [26], two main observations were introduced. First, the base station $(B S)$ in the direction of a mobile terminal's mobility will have a higher probability with respect to receiving a handoff from this MT. This probability decreases as the angle of the BS from the direction of the MT increases. When the 
angle is $90^{\circ}$ or higher, the probability is almost zero [26]. The second observation is that the smaller the estimated time for a MT to move into a particular cell, the higher the probability for that MT to be handed-off at that cell. The estimated time is calculated as the ratio of the distance of a MT from the BS and the velocity of the MT in that particular direction. This means for a stationary MT the bandwidth will only be allocated in the current cell, with no reservation made in neighboring cells. For a moving MT, the probability decreases as the distance between the MT and the BS increases. The probability of an MT visiting a particular cell was calculated based on velocity, distance, and direction. The bandwidth reservation is based on this probability with some thresholds used to determine the portion of the actual bandwidth that will be reserved. If the calculated probability is above the first threshold (high probability), then this probability is considered to be 1 and the bandwidth is reserved according to this probability. On the other hand, if the calculated probability is lower than the second threshold (low probability), then probability is considered to be 0 and the bandwidth is reserved according to this probability. Otherwise, portion of the bandwidth is reserved according to the calculated probability. This approach doesn't consider any previously known history of the users, which may enhance the performance of the network. Also, they didn't explain how to calculate the velocity, distance and direction of the mobile terminals effectively.

The idea of taking the mobility into account for CAC schemes was explained in details in [29]. It had been indicated that it is important to make the reservation at the correct time to save the reserved bandwidth. The suggested mobility-based 
channel reservation scheme is based on exchange the information about the reserved channels between neighboring cells periodically. Also, in [29] a new call bounding scheme to prevent the cell congestion by controlling the number of admitted new calls was suggested. Four handoff prioritized CAC strategies based on the introduced reservation and bounding schemes had been introduced. The authors in [29] did not consider the different requirements for bandwidth (voice, data, multimedia...). Also, the mobility history of the users had not been taken into account.

One of the recent predictive techniques was introduced in [33] where a distributed predictive channel-reservation scheme, called the road-map-based channel-reservation scheme (RMCR), and a call admission control algorithm were proposed. They also assume that the base stations have road-map information and the mobile terminals have a global positioning system (GPS). Based on the location information of the MTs at two consecutive periods, the BSs estimate the speed and moving direction of the MSs. Aslo, the BSs estimate the probability that the MSs will enter the neighboring cells based on their velocity and the road-map information stored in the BSs. The BSs then compute the amount of bandwidth to be reserved, based on such estimation. This technique is based on the accurate location information of the MS using the GPS which can be suitable in future mobile networks. This approach can be enhanced if the previous behavior history of the mobile user is included to estimate the required bandwidth in future movements. Also, in that approach, the authors did not consider soft handoff in code-divisionmultiple-access (CDMA) systems, in which an MS can communicate with two or more BSs in the region near the boundary simultaneously. 


\subsubsection{Prediction Using Previous Movements' History}

In [34], a predictive and adaptive resource reservation technique was proposed. In this technique, the base station (BS) calculates the required bandwidth to be reserved for handoff calls from neighboring cells. The mobility of the mobile user is estimated using a history of user movements recorded in each cell. Using this information, the scheme predicts the handoff time and the amount of bandwidth that should be reserved. The BS adjusts the amount of reserved bandwidth by changing the size of the estimation window, depending on the handoff failures recorded. The basic disadvantage of this approach is that they consider only the one dimensional case of the cells (like a car on a straight line) while the two dimensional case is a more realistic (as shown in Fig. 2.2). Also, they didn't consider different types of users' patterns (like pedestrians and stationary mobile terminals).

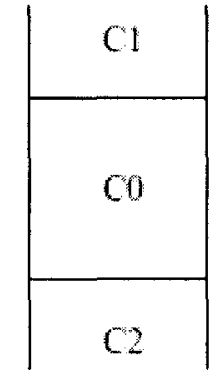

a) One dimensional case

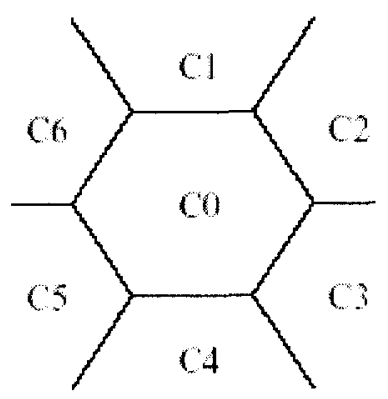

b) Two dimensional case

Fig. 2.2 Two different cellular environments

In $[27,35,36]$, a mobility-based predictive algorithm for $\mathrm{CAC}$ was presented. This algorithm is motivated by computational learning theory, which has shown that prediction is synonymous with data compression [27]. The proposed mobility 
prediction scheme is based on the character-based version of Ziv-Lempel algorithm. The sequence of events (new call, handoff or end of call) during the lifetime of a call corresponds to a substring in the Ziv-Lempel algorithm. The mobility database of every mobile terminal at a specific time holds a mobility trie, which is a probability model corresponding to that of the Ziv-Lempel algorithm. Each node except for the root in the mobility trie preserves the relevant statistics that can be used to predict the probability of following events [27]. As in data compression, the mobility trie of the mobile terminal is built in an on-line fashion. When a mobile user requests a new call, the predictor sets the current node to the root of the trie according to the identity of the mobile terminal, the cell it is in, and the current time, and calculates the probabilities of all possible events of this mobile terminal [27]. Upon recording an actual event of the mobile terminal, the predictor walks down the trie and is ready for the next prediction. When an event is not in the mobility trie, a prediction fault is generated and the trie is updated accordingly [27].

The main advantage in that approach is the prediction of the next cell and the time at which the handoff will occur. Also, the prediction is done for each individual user, which makes it more accurate. On the other hand, there are some limitations in that approach. It is assumed that there is no soft handoff, which makes this approach not suitable for future mobile telecommunication. Also, the delay sensitive applications are not considered, which is not suitable for real time multimedia applications in mobile networks. Besides, the approach requires from every base station to reserve the information about all users, which is not suitable for real mobile networks. 
The proposed technique in [28] is more suitable for multimedia and real time applications. The technique is called PR-CAT4. The call admission control and the resource reservation algorithms for PR-CAT4 are shown on Appendix E. PC-CAT4 technique is based on the mobility graph to predict the next cell (as shown in Fig. 2.3). It is also based on the 2-tire cell structure (as shown in Fig. 2.4) to determine the amount of the bandwidth to be reserved. Each node in the graph represents a visited cell. Edges represent the directions of the mobile user. In the proposed algorithm, the prediction of the next base station (cell $M$ ) is based on the current base station (cell $K$ ), the previous base station (cell $I$ ) and the number of times the client has moved from the current cell to the next cell. Then, the probability of the mobile user to move from cell $I$ to cell $M$ through cell $\mathrm{K}$ is calculated.

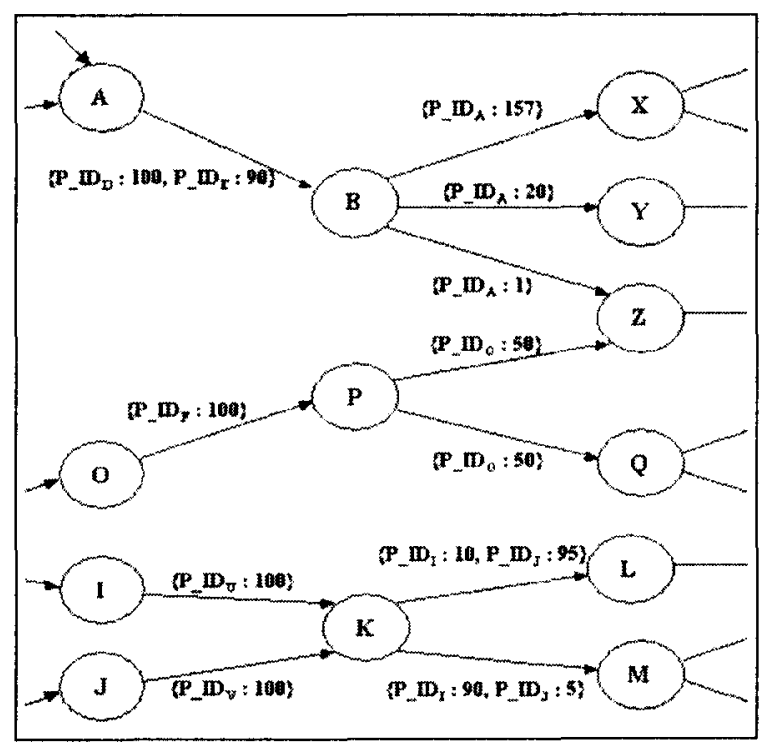

Fig. 2.3 An example of the mobility graph proposed in [28] 


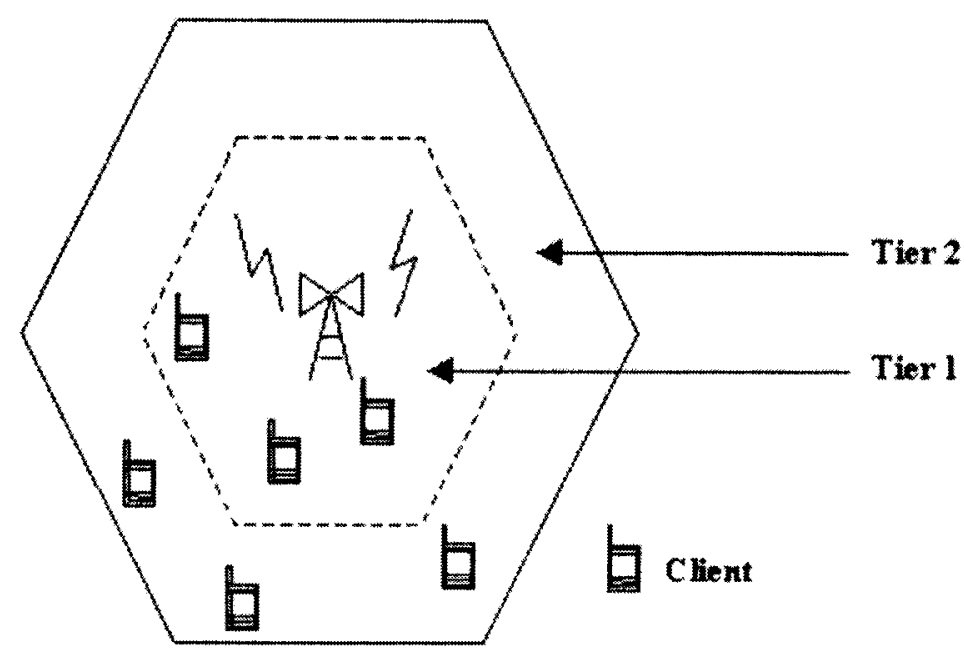

Fig. $2.4 \quad$ 2-Tire cell structure proposed in [28]

One of the disadvantages in this approach is that only short paths (previous, current, and next base stations) are considered, which limits the prediction to the next base station only. Also, the schemes presented in [28] did not consider any information related to the time at which these movements were performed or for how long the user stayed at each base station.

One of the most recent approaches for CAC and RR was introduced in [31, 32] which use a road topology based (RTB) mobility prediction. As in [32], the RTB approach assumes that the base stations have road-map information and the mobile terminals have a positioning system. The difference here is that they use the previous history of handoffs for the mobile users passed through a base station to estimate the time of handoff for the current user in that base station. They introduce a novel predictive reservation scheme that utilizes knowledge of road topology, in addition to 
positioning information. They develop an adaptive bandwidth reservation scheme that dynamically adjusts the reservation at each base station according to both incoming and outgoing handoff predictions generated using the mobility prediction technique. One of the main advantages of the RTB approach is that it considers also the irregular shapes of the cells which make it more realistic. Also, the authors consider both estimated incoming and outgoing handoff calls to estimate the available bandwidth for reservation which maximize the efficiency of this approach. This approach can be enhanced if they consider also the different periods of time during the day in the prediction technique.

\subsection{Limitations of Existing CAC and RR Techniques}

From all of the previous techniques, we can conclude that we still need a robust $\mathrm{CAC}$ and $\mathrm{RR}$ technique that can utilize efficiently the limited available radio resources and at the same time reduces the CDP and the CBP as much as possible. The limitations of most of the previously proposed techniques can be summarized into three main points. First, the accuracy of the prediction needs to be improved in a suitable way to reflect the real behavior of the mobile user. For example, the behavior of the user in the morning may be totally different comparing with his/her behavior in evening. Second, the time of service requests is an important factor to avoid the reservation overhead in the limited bandwidth. For example, the user may stay at the same cell for long time and we still reserve unnecessary bandwidth in the neighbor cells. Third, all of the predictive techniques in the existing literature (to the authors' knowledge) use the prediction at either the level of an individual user (local mobility model) or the level of a group of users (global mobility model). None of these 
techniques consider the combination between these two levels in a hierarchical structure to enhance the prediction accuracy.

Also, all the existing $\mathrm{CAC}$ and $\mathrm{RR}$ schemes proposed by researchers in this area deal with one type of mobile networks (such as cellular networks) and none of these schemes was designed for the wireless heterogeneous networks (WHN) which integrates more than one type of mobile networks.

To overcome these limitations we have designed, implemented, and evaluated new mobility-based predictive call admission control and resource reservation techniques for two different architectures of mobile networks: 1) cellular networks, and 2) wireless heterogeneous networks (WHNs) which integrate cellular networks and wireless local area networks (WLANs). The new techniques have more prediction capabilities, and with the idea of using the combination of local and global mobility models in a hierarchical structure. These details of these techniques are explained in the next chapters. 


\section{CHAPTER III}

\section{PREDICTIVE CAC AND RR FOR NEXT- GENERATION CELLULAR NETWORKS: PCAC-RR}

This chapter addresses the problem of resource reservation and call admission control in wireless cellular networks. A new user mobility oriented predictive scheme called PCAC-RR is proposed for call admission control and resource reservation. The main goal is to reduce the call dropping probability and the call blocking probability, and to maximize the bandwidth utilization. By analyzing the previous movements of the mobile users, we generate local and global mobility profiles for mobile users, which are utilized effectively in the prediction of the future path of a mobile user.

\subsection{PCAC-RR Methodology}

The predictive based call admission control and resource reservation technique (PCAC-PR) methodology is mainly based on the generation of two types of users' mobility profiles (models). The key here is how we can effectively record and analyze the previous behavior of the mobile users to generate the mobility profiles. These profiles should contain the frequent paths and its associated probability. Frequent paths will be used to predict the next movements of the mobile users. The generated profiles for each user will contain useful information related to the behavior of this user. This information includes the visited paths, the day period at which each 
path was visited, estimated time length of the path, and the probability of visiting that path. Data mining techniques are used to extract this information. Also, the proposed methodology provides a suitable QoS guarantee for mobile communications.

The first type of the mobility profiles is called the local mobility profile. This profile is generated locally for each user by his mobile host $(M H)$. The MH will have the responsibility to collect the movement data of a user that uses this MH. This data is used to build the mobility profile of users and will be continually updated based on users movements. The second type of the mobility profiles is called the global mobility profile. This profile is generated by each Base Station (BS). The BS will have the responsibility to collect the movements' data of the users passing through the cell that contains this base station. This global profile will be used if the local profile gives low prediction accuracy. To update the local and global profiles, we currently usc direct updating where we update the profiles according to the new collected data after a period of time.

Figure 3.1 shows an overview of the system architecture. Every time the user enters a new BS, the MH will start to predict the next base stations according to the current local profile of this user. Once a conversation has started, this information about the next cells will be sent to the current BS (with additional information about the expected handoff time) before the handoff process. The current BS will use its own global profile if the MH couldn't predict the next cell (for the case of new visited base stations or rarely visited base stations). This methodology takes the benefits of 
both local and global profiles, where the combination enhances the accuracy of the prediction.

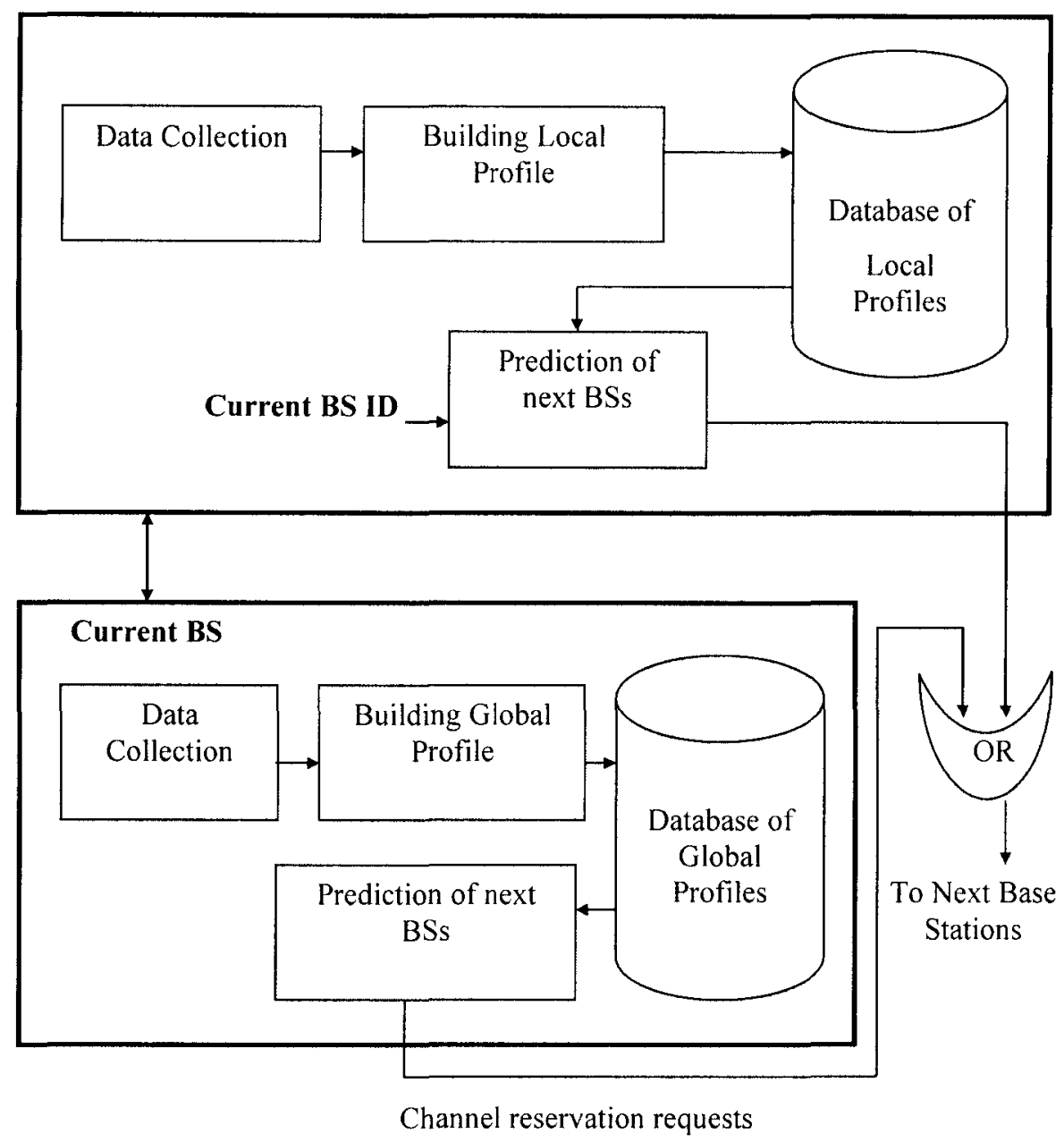

Fig. 3.1 The system architecture supported by the PCAC-RR technique

The PCAC-RR methodology assumes that we have suitable memory space and computational resources in the mobile handset, which are now available using VLSI technology. We assume that there will be intelligent software in the mobile 
handset responsible for collecting and mining the data locally. This software could be implemented using the Java language, which is supported in the new generations of the mobile handsets. The used data mining techniques will be built effectively to save the power consumed during the computation process. Also, the service providers could easily update this software itself remotely to enhance the service.

\subsection{Architecture of Cellular Networks Supported by PCAC-RR}

The general architecture of the cellular mobile networks is shown in Fig. 3.2. Each cell has a Base Station $(B S)$ that can communicate with the Mobile Host $(M H)$ inside this cell. Mobile Switching Center (MSC) controls a number of base stations connected to it. The mobile user is supposed to move between these cells. If the mobile user is moving from cell A to cell B and there is an ongoing call, then this call has to be handed over to the other cell in an efficient way to prevent the interruption in the service. This process of switching a call is called handoff. If there is no available bandwidth to reserve this call in the next cell, this call may not complete and in this case we will have a dropped call. Also, if there is a new incoming call and there is no available bandwidth to serve this call in the current cell, this call may be rejected and in this case we will have a blocked call. From the nature respective, it is mandatory to continue ongoing calls and block the new calls if received. Dropping the ongoing calls is more undesired action than blocking the new calls. Hence, handoff calls generally have a priority over the new calls [7]. 


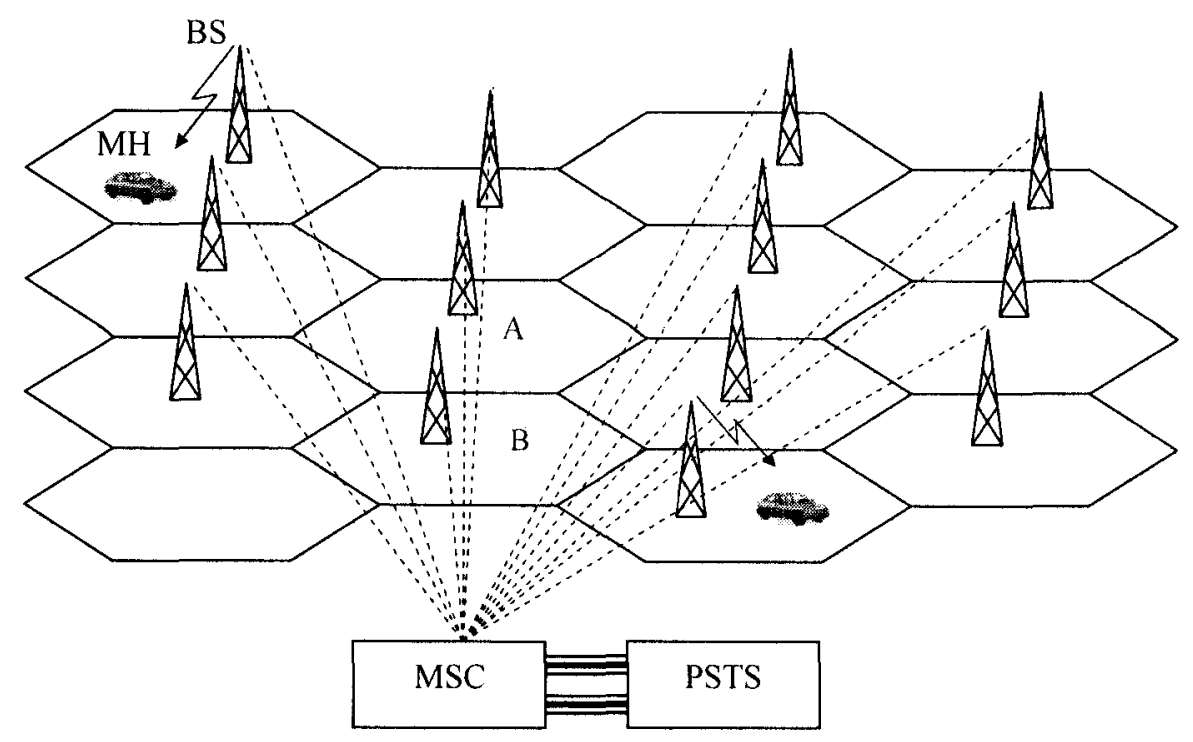

Fig. 3.2 Cellular Networks Architecture

The basic idea of our research is to develop mobility based predictive call admission control and resource reservation technique (PCAC-PR) to enhance the performance of wireless mobile networks [37]. In PCAC-RR technique, we use data mining approach to analyze the previous behavior of the cellular mobile users and to generate the mobility profiles (models) [38]. These models are used to predict the future paths of the mobile users. The mining process is distributed through the mobile hosts as well as the base stations to generate both local (personal) and global (general) mobility profiles $[37,39]$.

There are three main characteristics of the proposed PCAC-RR technique which make it superior over the other techniques:

1. The time of service request is considered as an important factor in prediction technique. 
2. This technique uses a hierarchical structure of local and global mobility profiles which improves the prediction accuracy.

3. This technique predicts the future path of the user (not only the next cell).

\subsection{Inductive Learning through Mobile Data Mining}

PCAC-RR technique is an inductive learning based technique, where a generalization is obtained from a set of samples and it is formalized using models [40]. Inductive learning through mobile data mining is used in this technique as the mobility data of the mobile users is collected and analyzed to generate the mobility profiles that can be used to predict the next movements of the mobile users. Two types of profiles are generated: local mobility profiles for the individual users and global mobility profiles for the group of users at given BS. The local mobility profile is generated locally for each user by his $\mathrm{MH}$. The $\mathrm{MH}$ will have the responsibility to collect the movement data of a user that uses this MH. The data is used to build the mobility profile of users and the profile will continually be updated based on new users movements. The global mobility profile is generated by each BS. The BS has the responsibility to collect and mine the movements' data of the users passed through it. These two types of profiles and their coordination are the three basic components of a distributed model that will be used by the CAC and RR schemes to predict the future paths of the mobile users. 


\subsubsection{Generation of Local Mobility Profiles}

The user movements between the cells in the mobile networks can be translated into a set of paths where each of these paths contains a sequence of cells IDs. For example the path that contains $m$ cells IDs can be written as:

$$
\operatorname{Path}_{i}=\left\{B S_{1}, B S_{2}, \ldots, B S_{m}\right\}
$$

where $B S_{j}$ is the ID of a base station in that path.

The profiles will contain a set of sequences in the form of paths that are most likely to be visited (frequent paths). These frequent paths are generated by mining these sets of sequences [41]. The sequence mining task can be defined in general as a task that aims to discover a set of attributes, shared across time among a large number of objects in a given database $[42,43]$. The Local Profile (LP) that contains $n$ paths can be defined as:

$$
L P=\left\{\text { Path }_{1}, \text { Path }_{2}, \ldots, \text { Path }_{n}\right\}
$$

Local mobility profiles are generated separately for each mobile user. These local mobility profiles are built based on the data colleted by each mobile handset. The software in the MH collects the user's movement data. The MH mines the collected data to generate the local profiles. Data collection and data mining are basic phases in forming local and global mobility profiles. In this part we will focus on the explanation of the local mobility profiles.

\subsubsection{Data Collection for Local Profiles}

Data collection is performed by the MH during the navigation of the mobile user. Each time the mobile user enters a new cell, the data collection software records the ID of this new cell and the times at which the visit was started and ended. An 
example of the structure for data collected by one of the mobile hosts is shown in Table 3. 1.

Table 3.1 Example of the collected data in the $\mathrm{MH}$

\begin{tabular}{|c|c|c|}
\hline ID of Visited BS & Visit Start Time & Visit End Time \\
\hline $\mathrm{BS}_{1}$ & $\mathrm{TS}_{1}$ & $\mathrm{TE}_{1}$ \\
\hline $\mathrm{BS}_{2}$ & $\mathrm{TS}_{2}$ & $\mathrm{TE}_{2}$ \\
\hline$\ldots$ & $\ldots$ & $\ldots$ \\
\hline $\mathrm{BS}_{\mathrm{n}}$ & $\mathrm{TS}_{\mathrm{n}}$ & $\mathrm{TE}_{\mathrm{n}}$ \\
\hline
\end{tabular}

\subsubsection{Discovering of Local Paths}

The discovering of the local paths is performed by the $\mathrm{MH}$ by using frequent sequence mining techniques where paths are formalized as sequences of symbols (ID's for base stations). The main idea is to mine the collected data to generate the local profile for each user. We introduce a hypothesis that user behaviors (and therefore user profiles) are not the same for different periods of the day. Therefore we propose dividing the day into several intervals. These intervals will be determined generally for the users according to the known rush hours, general work hours, etc. Fore example, we can divide the day into the following six intervals: $T_{1}$ (morning rush hours: [6:00 - 9:00]), $\mathrm{T}_{2}$ (morning-noon: [9:00 12:00]), $\mathrm{T}_{3}$ (after noon [12:00 5:00]), $\mathrm{T}_{4}$ (evening rush hour: [5:00-6:00]), $\mathrm{T}_{5}$ (evening: [6:00 - 12:00]), and $\mathrm{T}_{6}$ (after midnight: $[12: 00-6: 00])$. The local profile contains the possible paths with associated probabilities for each time interval. This means that a path may have different probabilities for different intervals in a day. Each path in the local profile is described by a sequence of base stations. Therefore it is necessary to transform the collected data in the $\mathrm{MH}$ into a sequence of symbols, where each symbol represents a 
base station. Every path can be transformed into a sequence of base stations ID for each recorded visit.

Figure 3.3 shows an example of using the time slots of 2 minutes $(\Delta t=2$ minutes) in the local profiles. In this example, the mobile host started to visit base station $\mathrm{BS}_{2}$ at time 1:02 $\mathrm{pm}$ and the visit ended at time 1:08 pm. Using $\Delta \mathrm{t}=2$ minutes, we can say that this mobile user visited $\mathrm{BS}_{2}$ for three consecutive time slots. The generated path based on this time slot is as shown in Figure 3.3. This type of path representation contains implicit information about speed of transition between the cells. The information will be very useful in predicting when the handoff will happen. Proposed model of paths, which include time component, will enhance the overall performance by preventing unnecessary early bandwidth reservation. This is an important advantage of the PCAC-RR approach, where we can predict not only the next base stations, but also the time at which the handoff will happen. In addition our approach can also predict, based on past history, how long a mobile user will stay in the next cells.

Collected Data

Using $\Delta t=2 \mathrm{~min}$

\begin{tabular}{|c|c|c|}
\hline Visited BS ID & Visit Start Time & Visit End Time \\
\hline $\mathrm{BS}_{1}$ & $1: 00$ & $1: 02$ \\
\hline $\mathrm{BS}_{2}$ & $1: 02$ & $1: 08$ \\
\hline $\mathrm{BS}_{3}$ & $1: 08$ & $1: 12$ \\
\hline
\end{tabular}

\begin{tabular}{|l|}
\hline $\mathrm{BS}_{1}$ \\
\hline $\mathrm{BS}_{2}, \mathrm{BS}_{2}, \mathrm{BS}_{2}$ \\
\hline $\mathrm{BS}_{3}, \mathrm{BS}_{3}$ \\
\hline
\end{tabular}

$\left\{\mathrm{BS}_{1}, \mathrm{BS}_{2}, \mathrm{BS}_{2}, \mathrm{BS}_{2}, \mathrm{BS}_{3}, \mathrm{BS}_{3}\right\}$

Transformed

into a path

Fig. 3.3 Path generation process for local paths 
To determine when we terminate the current path, and start to allocate the visited cells in a new path, we consider the following three conditions:

1. We reach the edge of the current time interval.

2. The path length reaches a predefined maximum path length $\left(L_{\max }\right)$

3. The current cell was visited for a consecutive predefined number of times $\left(\mathrm{N}_{\max } ;\right.$ where $\left.\mathrm{N}_{\max }<\mathrm{L}_{\max }\right)$

If one of these conditions is satisfied (whichever is satisfied first), then the current path is terminated (end of the path) and we start to register the visited cells in a new path. The first condition ensures that we are still within the correct time interval. The second condition limits the path length to a predefined threshold. This will prevent the very long paths. The type of paths terminated under this condition express the movements with high speeds (for example movements on highways). The third condition is used to detect when the movements start to be within the same cell or when the speed has been decreased. In our implementation, we used $L_{\max }=5$ and $\mathrm{N}_{\max }=3$. This means that the path length may be 3,4 or 5 .

For each of the generated paths we calculate two types of probabilities. The first one is the overall probability for each path (during the whole day). This probability can be calculated from equation (1.1):

$$
P\left(\text { path }_{n}, m_{l}\right)=\frac{\sum_{j=1}^{N_{i}} \text { Num }\left(\text { path }_{n}, T_{j}, m_{l}\right)}{\sum_{j=1}^{N_{l}} \sum_{i=1}^{N_{p}\left(m_{l}\right)} N u m\left(\text { path }_{i}, T_{,}, m_{l}\right)}
$$

where 
$P\left(\right.$ path $\left._{n}, m_{l}\right): \quad$ is the probability of the mobile $m_{l}$ to go through the path path $_{n}$

Num $\left(\right.$ path $\left._{n}, T_{j}, m_{l}\right)$ : is the number of times for mobile $m_{l}$ to go through path during the time interval $T_{j}$

$N_{T}$ : is the number of time intervals

$N_{p}\left(m_{l}\right)$ : is number of stored paths for the mobile $m_{l}$.

The other type of probability is the probability of the path in a certain time interval . It could be expressed in terms of the probability of the path $p^{2} h_{n}$ for the given interval $T_{j}$ which is written as $P\left(\right.$ path $\left._{n}, m_{l} \mid T_{j}\right)$. This probability can be calculated from the equation:

$$
P\left(\text { path }_{n}, m_{l} \mid T_{l}\right)=\frac{\text { Num }\left(\text { path }_{n}, T_{j}, m_{l}\right)}{\sum_{i=1}^{N\left(m_{l}\right)} N u m\left(\text { path }_{i}, T_{i}, m_{l}\right)}
$$

The generated paths and their probabilities for a single user will be saved in the form shown in Table 3.2 .

Table 3.2 Example of the discovered local paths for a single user

\begin{tabular}{|c|c|c|c|}
\hline $\begin{array}{c}\text { Time } \\
\text { Interval (Ti) }\end{array}$ & Visited Paths ( path $_{k}$ ) & $\begin{array}{c}\text { Periodical Probability } \\
\mathbf{P}\left(\text { path }_{k} \mid \mathbf{T}_{i}\right)\end{array}$ & $\begin{array}{c}\text { Overall Probability } \\
\left.\text { P( } \text { path }_{k}\right)\end{array}$ \\
\hline \multirow{4}{*}{$\mathrm{T}_{1}$} & Path $_{1}=\left\{\mathrm{BS}_{1}, \mathrm{BS}_{2}, \ldots, \mathrm{BS}_{\mathrm{m}}\right\}$ & $\mathrm{P}\left(\right.$ path $\left._{1} \mid \mathrm{T}_{1}\right)$ & $\mathrm{P}\left(\right.$ path $\left._{1}\right)$ \\
\hline & Path $_{2}$ & $\mathrm{P}\left(\right.$ path $\left._{2} \mid \mathrm{T}_{1}\right)$ & $\mathrm{P}\left(\mathrm{path}_{2}\right)$ \\
\hline & $\ldots$ & & \\
\hline & Path $_{\mathfrak{n}}$ & $P\left(\right.$ path $\left._{n} \mid T_{1}\right)$ & $P\left(\right.$ path $\left._{n}\right)$ \\
\hline & . & & \\
\hline \multirow{4}{*}{$\mathrm{T}_{\mathrm{N}}$} & Path $_{1}$ & & . \\
\hline & Path $_{2}$ & & . \\
\hline & & & \\
\hline & Path $_{m}$ & & \\
\hline
\end{tabular}




\subsubsection{Building Local Mobility Profiles}

The discovered paths are tested according to the periodical and the overall probabilities to determine the set of paths to be considered as frequent paths during the given time interval and to assign a probability called associated probability to each frequent path. This probability determines the amount of the required bandwidth to be reserved in every cell belonging to that path. The detailed algorithm of finding the frequent paths is shown in Appendix A. The following steps describe how to find the frequent paths:

1. For each time interval, we start to test probability of each path in the given time interval $P\left(\right.$ path $\left._{n} / T_{i}\right)$ with respect to a certain predetermined threshold $L T H_{l}$ (say 0.8).

2. If $P\left(\right.$ path $\left._{n} / T_{l}\right)>L T H_{l}$, then this path is considered frequent and associated probability of this path is equal to one $\left(P_{a s s o d}\left(\right.\right.$ path $\left.\left._{n}\right)=1\right)$.

3. If the path probability is not high enough $\left(P\left(\right.\right.$ path $\left.\left._{n} / T_{i}\right)<L T H_{l}\right)$, then the overall probability $P\left(p a t h_{n}\right)$ is tested instead of $P\left(p^{2} t h_{n} / T_{i}\right)$.

4. If $P\left(\right.$ path $\left._{n}\right)>\mathrm{LTH}_{2}$ (another certain predetermined threshold, say 0.6), then this path is considered frequent, and its associated probability will be $\operatorname{Passoc}\left(\right.$ path $\left._{n}\right)=P\left(\right.$ path $\left._{n}\right)$.

5. Otherwise, the associated probability will be $\operatorname{Passoc}\left(p_{a t h}\right)=P\left(\right.$ path $\left._{n} / T i\right)$ and the path is not frequent. 
At the end of this process, the paths are stored in a table called $L P\left(T_{i}\right)$ (Local Profile at time interval $T_{i}$ ) which will contain the predicted local paths as well as the corresponding associated probabilities.

The LP table corresponding to each time interval is stored locally in the mobile handset. These paths with their associated probabilities will be used for the proposed resource reservation and CAC algorithms. An example of the generated local profile for a user is shown in Table 3.3.

Table 3.3 Example of the local profiles for a single user covering different time intervals $T_{i}$

\begin{tabular}{|c|c|c|}
\hline $\begin{array}{c}\text { Time Interval } \\
T_{\mathbf{i}} \\
\end{array}$ & $\begin{array}{c}\text { Visited Paths } \\
\left(\text { path }_{n}\right)\end{array}$ & $\begin{array}{l}\text { The Associated Probability } \\
\text { P }_{\text {assoc }}\left(\text { path }_{\mathbf{n}}\right)\end{array}$ \\
\hline \multirow{4}{*}{$\operatorname{LP}\left(\mathrm{T}_{1}\right)$} & Path $_{1}=\left\{\mathrm{BS}_{1}, \mathrm{BS}_{2}, \ldots, \mathrm{BS}_{\mathrm{m}}\right\}$ & $\mathrm{P}_{\text {assoc }}\left(\mathrm{Path}_{1}\right)=1$ \\
\hline & Path $_{2}$ & $\mathrm{P}_{\text {assoc }}\left(\mathrm{Path}_{2}\right)=0.7$ \\
\hline & $\ldots$ & $\ldots$ \\
\hline & Path $_{k}$ &. \\
\hline$\ldots$ & $\ldots$ & $\ldots$ \\
\hline \multirow{4}{*}{$\operatorname{LP}\left(T_{N}\right)$} & Path $_{1(\mathrm{~N})}$ &. \\
\hline & Path $_{2(\mathrm{~N})}$ & . \\
\hline & $\ldots$ & $\ldots$ \\
\hline & Path $_{\mathbf{m}(\mathrm{N})}$ & . \\
\hline
\end{tabular}

\subsubsection{Global Mobility Profiles}

Global mobility profiles are generated and maintained by the base stations. The global profiles contain sequences of paths that are most likely to be visited by the users passed through the base station. Each of these paths contains a set of cells IDs. The global profile (GP) is defined as:

$$
G P=\left\{\text { Path }_{1}, \text { Path }_{2}, \text { Path }_{3}\right\}
$$

and

$$
P a t h_{i}=\left\{B S_{1}, B S_{2}, B S_{3}\right\}
$$


where $B S_{l}: \quad$ is the ID of the previous base station

$B S_{2}: \quad$ is the ID of the current base station where this profile was

generated and used

$B S_{3}: \quad$ is the ID of the next base station that is expected to be visited after $\mathrm{BS}_{2}$

Each BS is responsible for collecting the mobility information of the subscribers passed through this base station. This information in addition to the known information about topology of the served area is used to generate the most frequent paths that can be used to predict the next movements of the user. Data collection and data mining are basic phases in forming model of global profiles.

\subsubsection{Global Data Collection}

Data are collected by each BS from the mobile hosts connected to this BS. The current BS at which the user is currently located will record the previous and the next base stations for this user as well as the times at which the visit was started and ended at the current BS. This base station will record this type of information for each user passed through it. The BS will be responsible to mine all of these paths to build the global profile for the given cell.

\subsubsection{Discovering of Global Paths}

As for the local profile, we have two types of probabilities for each generated path in the current cell. The overall probability of the global path is given by:

$$
P\left(\text { path }_{n}\right)=\frac{\sum_{k=1}^{N_{m^{\prime}}} \sum_{j=1}^{N_{i}} N u m\left(\text { path }_{n}, T_{1}, m_{k}\right)}{\sum_{k=1}^{N_{n}} \sum_{j=1}^{N_{7}} \sum_{i=1}^{N_{p}\left(m_{k}\right)} N u m\left(\text { path }_{i}, T_{,}, m_{k}\right)}
$$


where $N_{m}$ is the number of mobile users, and the path here is the global path.

The other probability is the path probability for the given time interval $T_{j}$ which is given by:

$$
P\left(\text { path }_{n} \mid T_{j}\right)=\frac{\sum_{k=1}^{N_{m}} N u m\left(\text { path }_{n}, T_{j}, m_{k}\right)}{\sum_{k=1}^{N_{m}} \sum_{i=1}^{N_{0}\left(m_{k}\right)} \operatorname{Num}\left(\text { path }_{i}, T_{j}, m_{k}\right)}
$$

The process of building the global mobility profiles will be similar to the process described for local models in Section 3.3.3, but this time is specified from the point of view of the base stations. Also, BS will have a table called $G P\left(T_{i}\right)$ (Global Profiles at time interval Ti) that will contain all frequent global paths as well as the corresponding associated probabilities. The algorithm of finding the frequent paths in the global profile is shown in Appendix A.

\subsection{Predictive Resource Reservation Scheme (RR)}

We assume that we have two types of calls: Class-I and Class-II. The calls form Class-I are the real time calls (such as video and audio). This means that these calls are sensitive to time variations. The calls from Class-II are the non-real time calls (such as text and Image). This means the time factor is not so significant for the calls from Class-II. This classification of calls is an important parameter in the final implementation of the predictive resource reservation scheme.

The RR scheme uses the frequent paths computed earlier with their associated probabilities to reserve the suitable bandwidth, which will be used during the call handoff. Each cell will have a portion of the bandwidth reserved for handoff calls. In order to make a reservation for the bandwidth, the cells have to monitor the available 
bandwidth at any time, which will be used for reservation. For each cell $C_{i}$ the available bandwidth at any $T_{j}$ is denoted as $B_{a}\left(C_{i}, T_{j}\right)$. The available bandwidth could be calculated if we know the following:

- $B_{t}\left(C_{i} T_{j}\right):$ the total bandwidth of cell $C_{i}$ at time $T_{j}$

- $B_{u}\left(C_{i}, T_{j}\right)$ : the estimated bandwidth $u$ sed by users in cell $C_{i}$ at time $T_{j}$

- $B_{r}\left(C_{i}, T_{j}\right):$ the bandwidth reserved by cell $C_{i}$ for handoff calls at time $T_{j}$

The available bandwidth in cell $C_{i}$ at any time is calculated from equation (1.5):

$$
B_{a}\left(C_{i}, T_{j}\right)=B_{t}\left(C_{i} T_{j}\right)-B_{u}\left(C_{i}, T_{j}\right)-B_{r}\left(C_{i}, T_{j}\right)
$$

The service providers determine the total bandwidth assigned for each cell during the design phase. The bandwidth used by the users in cell $C_{i}$ at the expected handoff time $T_{h}$ can be estimated easily from the bandwidth used at the current time. If there is a call, the required amount of bandwidth to be reserved in the other cells have to be calculated to handle the handoff operation. The expected time at which the handoff will happen has to be taken into account too. Suppose that there is a call in cell $C_{i}$ at time $T_{j}$, the proposed The algorithm of the RR scheme is as shown in Appendix B. The resource reservation scheme will be executed as follows:

1. Start to predict the future paths by looking at $\mathrm{LP}\left(T_{j}\right)$ (the local profile).

2. If there is at least a predicted frequent path in the local profile, then the bandwidth reservation is performed based on this local profile.

i. For each cell $C_{k}$ in the local paths, we start to estimate the handoff time $\left(T_{h}\right)$ using the known time slot $\Delta t$.

ii. Once we find $T_{h}$, we start to check the available bandwidth at $T_{h}$. The available bandwidth could be calculated from equation (1.5). 
iii. The required amount of bandwidth $B_{\text {req }}$ to be reserved is determined as a portion from the actual required bandwidth $B_{\text {actual }}$ according to the associated probability of the path using the following equation:

$$
B_{\text {req }}=B_{\text {actual }} * P_{a}\left(\text { path }_{n}\right)
$$

iv. If there is enough bandwidth available at time $T_{h}$, then the amount of bandwidth to be reserved will be $B_{\text {req }}$ and the available and the reserved bandwidths are updated.

v. If there is no sufficient bandwidth available and the call is from Class-I, then this call can borrow some amount $\left(B_{\text {borrow }}\right)$ form the Class-II calls in that cells.

vi. Otherwise the amount of bandwidth to be reserved will be the available bandwidth plus $\mathrm{B}_{\text {borrow }}$

3. If no local frequent paths could be predicted using $\operatorname{LP}\left(T_{j}\right)$, then we have to access the global profile, $\operatorname{GP}\left(T_{j}\right)$, and use the global paths in a way similar to the one described in the local profiles (step 2) to reserve the required bandwidth.

\subsection{Predictive Call Admission Control Scheme (PCAC)}

The admitted call may be a new call or a handoff call. Each of these categories of calls will be handled in a different way taking into account that the handoff calls will have a priority over the new calls. The algorithm of the PCAC scheme is shown in Appendix B. For new calls from Class-I, PCAC scheme will check the available BW in the current cell $C_{i}$ (at which the admission is performed) as well as the available BWs in the next expected cells in the predicted frequent paths with high associated probabilities $\left(P_{\text {assoc }}\left(\right.\right.$ path $\left.\left._{n}\right)\right)$. If each of these cells has available bandwidth $(B W)$ to cover this new call at the next time step, then the call is accepted and the required BW 
is allocated. Otherwise the call is blocked. For the new calls from Class-II, PCAC scheme checks only the available BW at the current cell. If there is an available BW, then the call is accepted and a BW is allocated ( $\left.B_{\text {accepted }}\right)$.

Handoff calls can allocate $\mathrm{BW}$ from the reserved $\mathrm{BW}$ and from the available BW. Suppose that the admission for a handoff call is performed at the cell $\mathrm{Ci}$ and at the time interval $\mathrm{T}_{\mathrm{j}}$. The calls from Class-I will be accepted if the required $B W$ $\leq\left[\operatorname{Br}\left(C_{i}, T_{j}\right)+B_{a}\left(C_{i}, T_{j}\right)\right]$. Otherwise the call is dropped. For handoff calls from ClassII, the call will be accepted if there is BW available in the reserved or the available BWs.

PCAC approach maximizes the probability of completing the call without dropping it during the user navigation through the cells. 


\section{CHAPTER IV}

\section{MULTIDIMENSIONAL SEQUENTIAL MINING FOR MOBILE NETWORKS: MOBILEPREFIXSPAN}

In this chapter we explain the details of the data mining technique, called MobilePrefixSpan, which we have developed to analyze the information collected from the mobile users and extract the mobility patterns. MobilePrefixSpan technique is a modified version of sequence mining technique PrefixSpan [44, 45] and its modified version for multidimensional sequences UNISEQ [46]. The spatial, temporal, and usage information are used in MobilePrefixSpan to extract the movement patterns of mobile users. The goal of MobilePrefixSpan technique is to extract the movement patterns of the mobile users using the collected information.

\subsection{Input and Outputs in MobilePrefixSpan:}

Fig. 4.1 shows a block diagram that describes the inputs and outputs for the MobilePrefixSpan technique. There are three types of information that are collected and used to generate the mobility patterns:

1. Spatial Information: this indicates the location of the mobile user at every recording time. This information is collected by recording the ID of each base station (BS). 
2. Temporal Information: this indicates the time and the day information collected during the navigation of the mobile users. At each recorded movement we record also the day and the time of the day at which this movement was performed.

3. Usage Information: this indicates the type of the services (or the call sessions) that has been used. We assume that we have two types of services (call sessions): Class-I and Class-II. The calls from Class-I are the real time calls (such as video and audio). It means that these calls are sensitive to time variations. The calls from Class-II are the non-real time calls (such as text and image), where the time factor is not significant. This classification of calls is an important parameter in the implementation of the predictive resource reservation scheme.

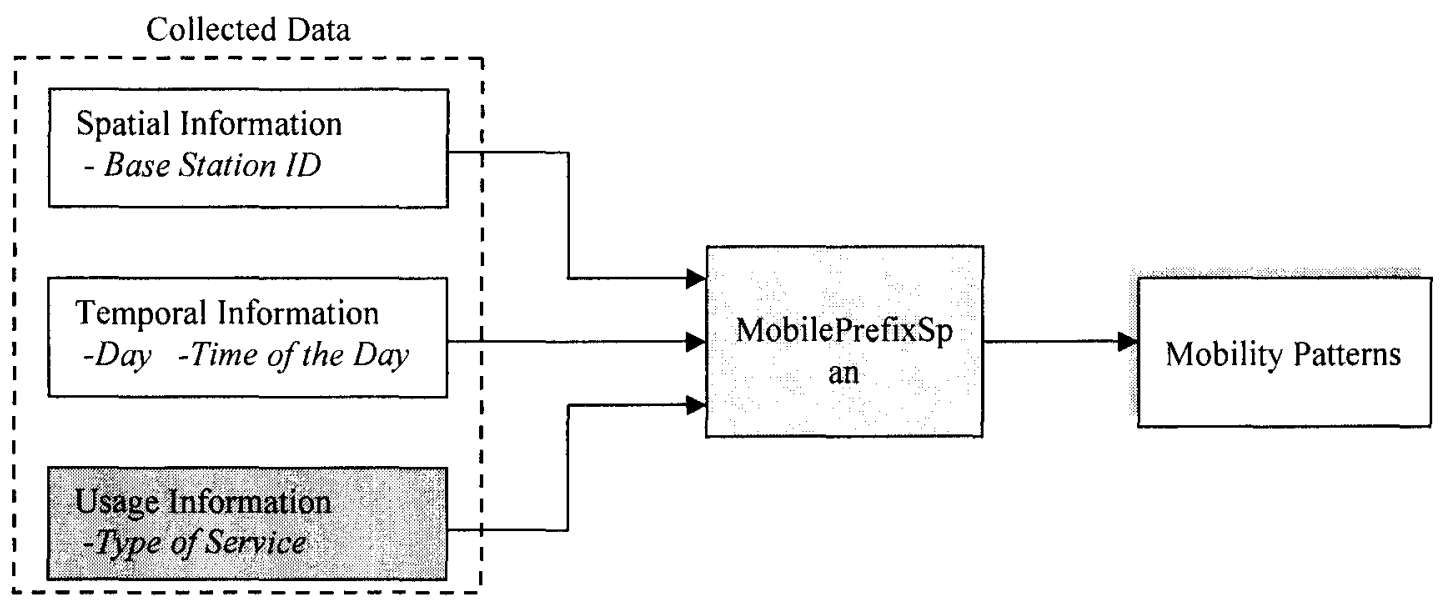

Fig. 4.1 Inputs and outputs for MobilePrefixSpan technique 


\subsection{Problem Definition of Sequence Mining in the Mobile Network}

\section{Environment}

Data mining extracts implicit, previously unknown, and potentially useful information from datasets. Many approaches have been proposed to extract information. One of the most important ones is mining sequential patterns. The sequential pattern mining problem was first introduced by Agrawal and Srikant in [41]: "Given a set of sequences, where each sequence consists of a list of elements and each element consists of a set of items, and given a user-specified min support threshold, sequential pattern mining is to find all of the frequent subsequences, i.e., the subsequences whose occurrence frequency in the set of sequences is no less than min support".

In this section, we give the basic definitions to formalize the problem of sequence mining in the mobile environment. These definitions for MobilePrefixSpan technique are based on the definitions that have been introduced in $[44,46,47]$.

\section{Definition 1:}

Let $I=\left\{i_{1}, i_{2}, \ldots, i_{n}\right\}$ be a set of items such that each item here represents a base station ID (or cell ID). An itemset $X$ is a subset of items, i.e., $X \subseteq B$.

\section{Definition 2:}

A sequence is an ordered list of itemsets. A sequence $S$ is denoted by $<s_{1} s_{2}$ $\ldots s_{k}>$ where $s_{j} \subseteq I$ for $l \leq j \leq k$. 
In our case each sequence represents an ordered set of base station (cells) IDs. For example, the sequence $\mathrm{S}_{1}=<\mathrm{C}_{1} \quad \mathrm{C}_{2} \quad \mathrm{C}_{3}>$ represents the transition of the mobile user from cell $C_{1}$ to $C_{2}$ and then to $C_{3}$.

\section{Definition 3:}

The number of instances of items in a sequence is called the length of the sequence.

For example, a sequence with length $k$ is called a $k$-sequence. For example the sequence $S_{1}=<\begin{array}{lllllll}C_{l} & C_{3} & C_{4} & C_{5} & C_{8}>\text { is a 5-sequence. }\end{array}$

\section{Definition 4:}

A sequence $\alpha=<\begin{array}{llll}a_{l} & a_{2} & \ldots\end{array} a_{n}>$ is called consecutive-subsequence of another sequence $\beta=<b_{1} \quad b_{2} \ldots b_{m}>$ and donated as $\alpha \subseteq \beta$ if all of the ordered itemsets in $\alpha$ appears in the exact same consecutive order in $\beta$.

This definition is needed for sequence mining in the mobile environment. Our problem does not only consider the order of visited cells, but also considers the path which contains consecutively ordered cells that are required to move from one cell to another. For example, the 5-sequence $\mathrm{S}_{1}=<\begin{array}{llllll}C_{1} & C_{3} & C_{4} & C_{5} & C_{8}\end{array}>$ has only the following consecutive-subsequences:

- Four Subsequences of length 2: $\left\langle C_{1} \quad C_{3}\right\rangle,\left\langle C_{3} \quad C_{4}\right\rangle,\left\langle C_{4} \quad C_{5}\right\rangle$, and $<C_{5} \quad C_{8}>$ 
- Three Subsequences of length 3: $\left\langle C_{1} \quad C_{3} \quad C_{4}\right\rangle,\left\langle C_{3} \quad C_{4} \quad C_{5}>\right.$, and $<\begin{array}{lll}C_{4} & C_{5} & C_{8}\end{array}$

- Two Subsequences of length 4: $\quad<C_{1} \quad C_{3} \quad C_{4} \quad C_{5}>$ and $<C_{3} \quad C_{4} \quad C_{5}$ $C_{8}>$

Here we didn't consider other subsequences which violate the consecutive ordering condition such as the subsequence $<C_{I} \quad C_{4} \quad C_{5}>$ where $C_{4}$ in our example never comes directly after $C_{l}$.

\section{Definition 5:}

A sequence dataset $S$ is a set of tuples $\langle$ sid, $s\rangle$, where sid is an identification of the sequence and $s$ is a sequence.

\section{Definition 6:}

A tuple $<$ sid, $s>$ is said to contain a sequence $\alpha$, if $\alpha$ is a subsequence of $s$ (i.e. $\alpha \subseteq s)$.

\section{Definition 7:}

The support of a sequence $\alpha$ in the sequence dataset $S$ is the number of tuples that contain $\alpha$, i.e. :

$$
\sup (\alpha)=\mid\{<\text { sid }, s>:(<\text { sid }, s>\in S) \wedge(\alpha \subseteq s)\} \mid
$$




\section{Definition 8:}

A multidimensional sequence $S$ is an ordered set of items (cells) that have common attributes and is represented in the form:

$$
S=\left\{d_{l}, d_{2}, d_{3}, \ldots, d_{n},<s_{l} s_{2} \ldots, s_{k}>\right\}
$$

Where

$d_{i}$ is the value of the attribute at the $i^{\text {th }}$ dimension.

$n$ is the number of dimensions(attributes) and

$s_{j}$ is the base stations IDs.

For example, we have the following three dimensions are used in our problem:

- $d_{l}$ : the period of the day. If we have four periods, then $d_{l} \in\{T 1, T 2, T 3$, T4\}.

- $d_{2}$ : the type of the day which can be a weekend or a working day, where $d_{2} \epsilon$ $\{Y, N\}$

- $d_{3}$ : the type of the type of service provided, where $d_{3} \in\{$ "Class-I", "ClassII", "Idle"\}.

In this case, the multidimensional sequence $S=\left\{T 2, N\right.$, Class-I, $\left.<C_{1} \quad C_{4} \quad C_{5}\right\}$ represents the transition of the mobile user (who has a Class-I call) from cell $C_{1}$ to $C_{4}$ and then to $\mathrm{C}_{5}$ at the second period of the day (T2) and during a working day (N). 


\subsection{MobilePrefixSpan Algorithm}

As we have mentioned earlier, MobilePrefixSpan technique is a modified version of the well-known sequence mining technique PrefixSpan $[44,45]$ and its modified version for multidimensional sequences UNISEQ [46]. To explain the details of the MobilePrefixSpan algorithm, let us assume that we have the multidimensional dataset shown in Table 4.1 that contains the generated sequences of one of the mobile users. As we can see in this example, there are two dimensions as defined in the previous sections. The first step is to reconfigure the dataset such that the multidimensional information is embedded in the sequences. The new sequences will be as shown in Table 4.2. This converts the problem to one-dimensional sequence mining problem. Here the first three elements in any of these sequences always refer to the multidimensional information. Once we have these new sequences we can start applying the MobilePrefixSpan technique where we do not consider a subsequence if all the items that represent these cells are not found in the consecutive order (as explained in Definition 4). This rule is not applied to the items that represent multidimensional information. This is because we can combine any of these dimensions together without including the remaining dimensions, while we can not ignore the in-between cells.

The detailed steps of MobilePrefixSpan algorithm are given in Appendix C. These steps are explained here with the help of an example using the converted sequences shown in Table 4.2. We will use in this example a minimum support value equal to 2 (min_sup=2) which represents the minimum number of repetitions for the 
sequence in the given dataset in order to be considered as a frequent sequence. We can start to apply the MobilePrefixSpan technique as follows:

0 . Apply the first scan to find all of the single-item frequent sequences (prefix).

- In our example, these frequent sequences are as shown in Table 4.3 with the corresponding supports which represent the number of repetitions for each of these sequences in the dataset.

- Each single-item frequent sequence is called a prefix and it will be corresponding to a subset of frequent sequences (or sequential patterns). In our case, we have 10 subsets of sequential patterns.

1. Find the projected dataset corresponding to the single-item frequent sequences.

- The projected dataset consists of postfix sequences which contains all the frequent items that follow the first occurrence of the prefix at any sequence.

- For example, if we consider the prefix $<\mathrm{T} 1>$ in our example, we will find that the projected dataset contains the following tow postfix sequences: $<\mathrm{Y}$ Class-I $\quad \mathrm{C}_{2} \quad \mathrm{C}_{3} \quad \mathrm{C}_{4} \quad \mathrm{C}_{5}>$ and \begin{tabular}{lllll}
\hline $\mathrm{Y}$ & $\mathrm{C}_{6}$ & $\mathrm{C}_{2}$ & $\mathrm{C}_{3}$ & $\mathrm{C}_{4}$
\end{tabular}$>$.

2. We continue by finding out the single-item frequent sequences in each projected dataset.

- In our example these will be $\langle\mathrm{Y}\rangle,\left\langle\mathrm{C}_{2}\right\rangle,\left\langle\mathrm{C}_{3}\right\rangle$, and $<$ $\mathrm{C}_{4}>$. 
3. Record the frequent sequences that have been found using this prefix and previous ones

- Now, we have four two-item frequent sequences in our example which are $\langle\mathrm{T} 1 \mathrm{Y}\rangle,\left\langle\mathrm{T} 1 \mathrm{C}_{2}\right\rangle,\left\langle\mathrm{T} 1 \mathrm{C}_{3}>\right.$, and $\left\langle\mathrm{T} 1 \mathrm{C}_{4}>\right.$.

4. Use each of these recorded frequent sequences as a prefix to find its projected dataset.

- For example, the projected dataset of the prefix $<\mathrm{T} 1 \quad \mathrm{C}_{2}>$ contains the following two postfix sequences: $<\mathrm{C}_{3} \quad \mathrm{C}_{4} \quad \mathrm{C}_{5}$ $>$ and $<\mathrm{C}_{3} \quad \mathrm{C}_{4}>$. In this case we will find that the single-item frequent sequences in this projected dataset are $\left\langle\mathrm{C}_{3}\right\rangle$, and $<$ $\mathrm{C}_{4}>$. Here, we can consider only one three-item frequent sequence which is $<\mathrm{T} 1 \quad \mathrm{C}_{2} \quad \mathrm{C}_{3}>$ but we can't consider $<\mathrm{T} 1$ $C_{2} \quad C_{4}>$ because $C_{4}$ doesn't follow $C_{2}$ directly in this case but it has to be through $\mathrm{C}_{3}$.

5. Repeat steps from 3 to 5.

- If we continue to find the projected dataset of the prefix $<\mathrm{T} 1$ $\mathrm{C}_{2} \quad \mathrm{C}_{3}>$ we will find that we have $\left\langle\mathrm{C}_{4} \quad \mathrm{C}_{5}>\right.$ and $\left\langle\mathrm{C}_{4}\right\rangle$. Here, $\left\langle\mathrm{C}_{4}>\right.$ is the only single-item frequent sequence and this generates the four-item frequent sequence $<\mathrm{T} 1 \quad \mathrm{C}_{2} \quad \mathrm{C}_{3} \quad \mathrm{C}_{4}>$. After that we will not find more frequent sequences using the prefix $<\mathrm{T} 1 \quad \mathrm{C}_{2} \quad \mathrm{C}_{3} \quad \mathrm{C}_{4}>$ because the projected dataset will have only the postfix $\left\langle\mathrm{C}_{5}\right\rangle$, and it does not satisfy the minimum support. 
- At this point we can say that we have found all of the frequent sequences (or sequential patterns) using the prefix $<\mathrm{Tl} \quad \mathrm{C}_{2}>$.

6. This process will continue through the dataset for all of the items until we find all of the sequential patterns.

The complete table of all the 10 subsets of the generated sequential patterns for this example is shown in Table 4.4. Each generated sequential pattern reflects the spatial, temporal, and usage information. For example, the sequential pattern $<\mathrm{T} 1$ $\begin{array}{lllll}\mathrm{Y} & \mathrm{C}_{2} & \mathrm{C}_{3} & \mathrm{C}_{4}>\text { can be interpreted as the multidimensional sequential pattern }\{\end{array}$ $\left.\mathrm{T} 1, \mathrm{Y},{ }^{*},<\mathrm{C}_{2} \quad \mathrm{C}_{3} \quad \mathrm{C}_{4}>\right\}$ where the first three items refer to the dimensions. In this example we can say that at the when the period $\left(T_{1}\right)$ of the day is the first period and during the weekend the sequence $<\mathrm{C}_{2} \quad \mathrm{C}_{3} \quad \mathrm{C}_{4}>$ is a frequent sequence.

As we can see from Table 4.4, we have generated all of the sequential patterns including the frequent sequences that contain only one cell ID. For the application of our problem, we will use only the frequent sequences of that contains at least two cells IDs. For example, we can not use the three-item frequent sequence $<\mathrm{T} 1 \quad \mathrm{Y} \quad \mathrm{C}_{3}>$ because it contains only the information that $\mathrm{C}_{3}$ is frequent in the first period and during the weekend, but it does not have any information about the transition from one cell to another which is the most important information that we are trying to discover.

Also, we introduce and apply what we will call last-item rule to simplify the algorithm. The last-item rule states that "when we calculate the support to any item there is no need to consider the sequences where that item appears as the last element in these sequences". This is because this item will not have any postfix in these 
sequences. This will automatically eliminates some items form the set of frequent items. For example, without applying the last-item rule we have $\left\langle\mathrm{C}_{5}>\right.$ with support of 2 as a frequent item as show in Table 4.3. We can see that in Table 4.4 we will not find any frequent sequences using $\left\langle\mathrm{C}_{5}\right\rangle$ as a prefix. By applying the rule, these results can be deduced form the beginning when we recalculate the support of $\left\langle\mathrm{C}_{5}\right\rangle$ to be 1 (without including the first sequence in Table 4.2) and therefore it will not be a frequent item. The stored datasets of all possible sequential patterns will be as shown in Table 4.5. As we can see in this database, we consider only the paths with at least two cells IDs.

Table 4.1 Multidimensional sequences dataset collected by mobile user

\begin{tabular}{|c|c|c|c|c|}
\hline \multirow[b]{2}{*}{ sid } & \multicolumn{3}{|c|}{ Dimensions } & \multirow[b]{2}{*}{ Sequence } \\
\hline & $\begin{array}{c}\text { Time } \\
\text { Interval }\end{array}$ & $\begin{array}{c}\text { Weekend } \\
\text { Day }\end{array}$ & $\begin{array}{l}\text { Type of } \\
\text { Service }\end{array}$ & \\
\hline 1 & $\mathrm{~T} 1$ & $\mathrm{Y}$ & Class-I & $\begin{array}{lll}<\mathrm{C}_{1} & \mathrm{C}_{2} & \mathrm{C}_{3}\end{array}$ \\
\hline 2 & T2 & $\bar{Y}$ & Class-I & $<\overline{C_{5}} \quad \mathrm{C}_{6}$ \\
\hline 3 & $\mathrm{~T} 1$ & $\bar{Y}$ & Class-II & $<\mathrm{C}_{6} \quad \mathrm{C}_{2}$ \\
\hline 4 & $\mathrm{~T} 2$ & $\mathrm{~N}$ & Idle & $\begin{array}{llll}<\mathrm{C}_{2} & \mathrm{C}_{7} & \mathrm{C}_{6} & \mathrm{C}_{2}\end{array}$ \\
\hline
\end{tabular}

Table 4.2 Conversion of Table 3 to one-dimension sequences dataset

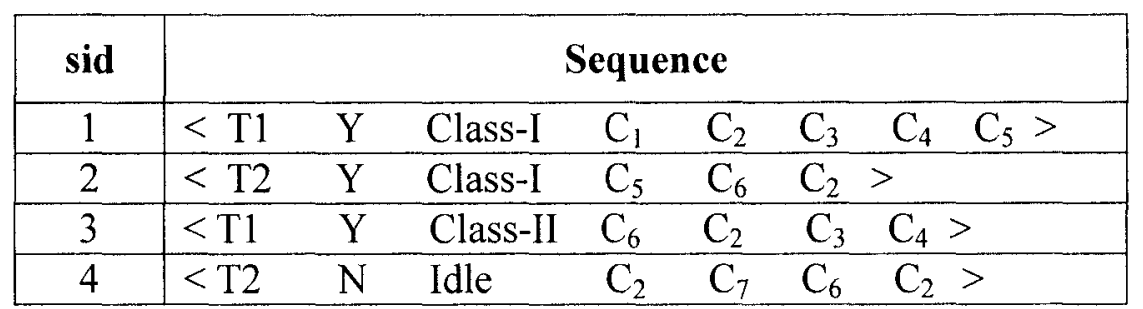

Table 4.3 Single-item frequent sequences

\begin{tabular}{|c|c||c|c|}
\hline $\begin{array}{c}\text { Single-item frequent } \\
\text { sequences }\end{array}$ & Support & $\begin{array}{c}\text { Single-item frequent } \\
\text { sequences }\end{array}$ & Support \\
\hline$<\mathrm{T} 1>$ & 2 & $<\mathrm{C}_{2}>$ & 4 \\
\hline$<\mathrm{T} 2>$ & 2 & $<\mathrm{C}_{3}>$ & 2 \\
\hline$<\mathrm{Y}\rangle$ & 3 & $<\mathrm{C}_{4}>$ & 2 \\
\hline$<$ Class-I $>$ & 2 & $\left.<\mathrm{C}_{5}\right\rangle$ & 2 \\
\hline & & $<\mathrm{C}_{6}>$ & 3 \\
\hline
\end{tabular}


Table 4.4 Complete Sequential Patterns for the given dataset

\begin{tabular}{|c|c|c|}
\hline Prefix & Postfix & Sequential Patterns \\
\hline$<\mathrm{T} 1>$ & $\begin{array}{lllll}<\mathrm{Y} & \text { Class-I } & \mathrm{C}_{1} & \mathrm{C}_{2} & \mathrm{C}_{3} \\
\mathrm{C}_{4} & \mathrm{C}_{5}> & & & \\
<\mathrm{Y} & \text { Class-II } & \mathrm{C}_{6} & \mathrm{C}_{2} & \mathrm{C}_{3} \\
\mathrm{C}_{4}> & & & & \end{array}$ & 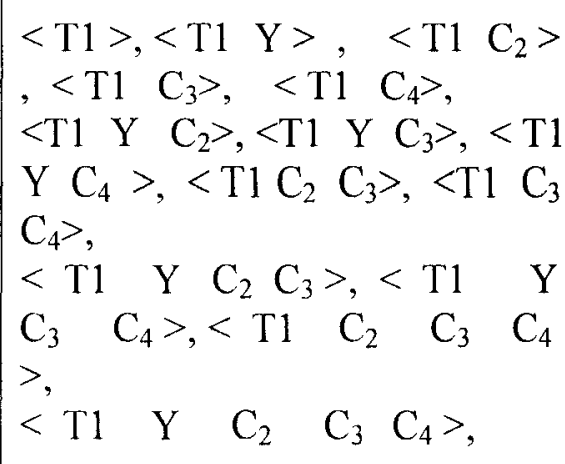 \\
\hline$<\mathrm{T} 2>$ & $\begin{array}{llllll}<\mathrm{Y} & \text { Class-I } & \mathrm{C}_{5} & \mathrm{C}_{6} & \mathrm{C}_{2} & > \\
<\mathrm{N} & \text { Idle } & \mathrm{C}_{2} & \mathrm{C}_{7} & \mathrm{C}_{6} & \\
\mathrm{C}_{2}> & & & & \end{array}$ & $\begin{array}{l}<\mathrm{T} 2>,<\mathrm{T} 2 \quad \mathrm{C}_{6}>,<\mathrm{T} 2 \\
\mathrm{C}_{2}> \\
<\mathrm{T} 2 \quad \mathrm{C}_{6} \quad \mathrm{C}_{2}>\end{array}$ \\
\hline$\langle\mathrm{Y}\rangle$ & $\begin{array}{lllll}<\text { Class-I } & \mathrm{C}_{1} & \mathrm{C}_{2} & \mathrm{C}_{3} & \mathrm{C}_{4} \\
\mathrm{C}_{5}> & & & & \\
<\text { Class-I } & \mathrm{C}_{5} & \mathrm{C}_{6} & \mathrm{C}_{2} & \\
<\text { Class-II } & \mathrm{C}_{6} & \mathrm{C}_{2} & \mathrm{C}_{3} & \mathrm{C}_{4}>\end{array}$ & 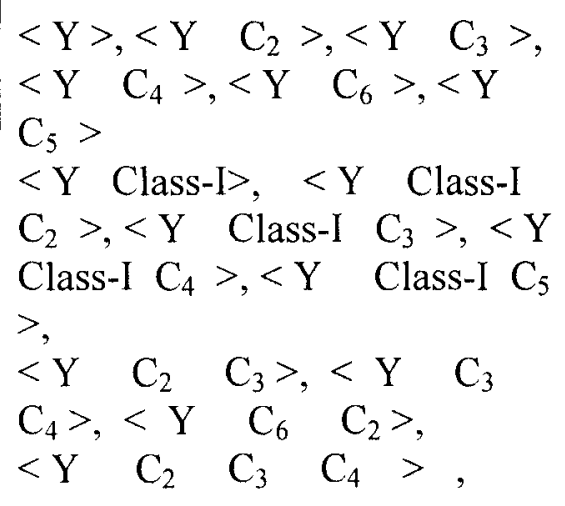 \\
\hline$<$ Class-I $>$ & 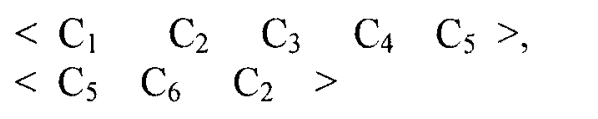 & $\begin{array}{l}<\text { Class-I }>,<\text { Class-I } \mathrm{C}_{2}>, \\
<\text { Class-I } \mathrm{C}_{5}>\end{array}$ \\
\hline$<\mathrm{C}_{2}>$ & 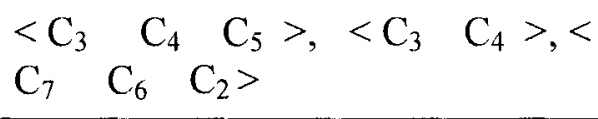 & $\begin{array}{l}<\mathrm{C}_{2}>,<\mathrm{C}_{2} \quad \mathrm{C}_{3}>,<\mathrm{C}_{2} \quad \mathrm{C}_{3} \\
\mathrm{C}_{4}>\end{array}$ \\
\hline$<\mathrm{C}_{3}>$ & $<\mathrm{C}_{4} \quad \mathrm{C}_{5}>, \quad<\mathrm{C}_{4}>$ & $<\mathrm{C}_{3}>,<\mathrm{C}_{3} \quad \mathrm{C}_{4}>$ \\
\hline$\left\langle\mathrm{C}_{4}\right\rangle$ & $<\mathrm{C}_{5}><>$ & $<\mathrm{C}_{4}>$ \\
\hline$\left\langle\mathrm{C}_{5}\right\rangle$ & $<\mathrm{C}_{6} \quad \mathrm{C}_{2}>$ & $<\mathrm{C}_{5}>$ \\
\hline$<\mathrm{C}_{6}>$ & $<\mathrm{C}_{2}>,<\mathrm{C}_{2} \quad \mathrm{C}_{3} \quad \mathrm{C}_{4}>,<\mathrm{C}_{2}>$ & $\left.<\mathrm{C}_{6} \quad \mathrm{C}_{2}\right\rangle$ \\
\hline
\end{tabular}


Table 4.5 Stored Sequential Patterns and corresponding support and confidence

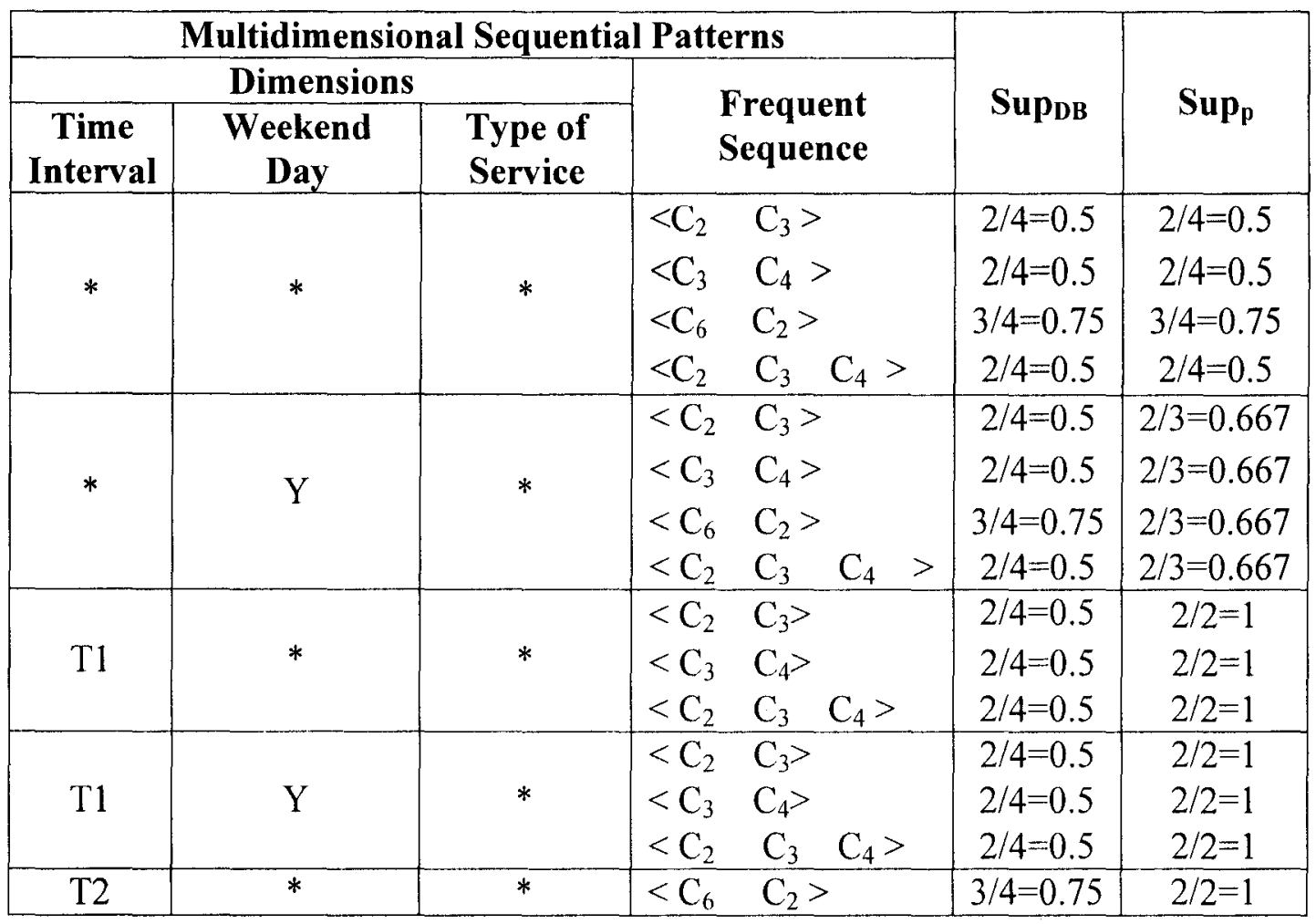

\subsection{Calculating the Support of the Generated Sequential Patterns}

As we can see in Table 4.5, we have calculated two types of supports for each frequent sequence. The first one is called the general support this refers to the support of the sequence in the database and is defined by:

$\sup _{D B}\left(S_{i}\right)$ is the support of the sequence $S_{i}=<s_{I} s_{2} \ldots s_{k}>$ in the dataset DB.

The support for a frequent sequence $S_{i}=<s_{1} \quad s_{2} \ldots s_{k}>$ is calculated by finding the ratio of the records in the dataset (without taking into account the dimensions) that contains the sequence $S$. For example, if we want to calculate the support of the frequent multidimensional sequence $\mathrm{S}_{1}=<\mathrm{C}_{6} \quad \mathrm{C}_{2}>$ shown in Table 4.4 , we will find that $S_{1}$ is appearing in three rows of the dataset shown in Table 4.1 (where the dataset 
size is 4 ), therefore the support of $S_{1}$ is equal to 1 (we write it as $\sup _{D B}\left(S_{1}\right)=0.75$ ). This is very helpful because it gives an indication about the repetition of the sequence in all the cases.

The second type of support is the projected support and this refers to the support of the sequence relative to the projected dataset according to the required dimensions and it is defied by:

$\sup _{p}\left(S_{i}, D_{j}\right) \quad$ is the projected support of the sequence $S_{i}$ relative to projected dataset from $\mathrm{DB}$ given by the dimensions $\mathrm{D}_{\mathrm{j}}$.

The projected support of a frequent multidimensional sequence $S_{i}=<_{1} \quad s_{2} \ldots$ $\mathrm{s}_{\mathrm{k}}>$ with dimensions $\mathrm{D}_{\mathrm{j}}=\left\{\mathrm{d}_{1}, \mathrm{~d}_{2}, \mathrm{~d}_{3}, \ldots, \mathrm{d}_{\mathrm{n}}\right\}$ is calculated by looking to the projected database that have the same dimensions $\left(d_{1}, d_{2}, d_{3}, \ldots, d_{n}\right)$ and find the ratio of the records that have the sequence $\left\langle s_{1} \quad s_{2} \ldots . s_{k}>\right.$ in this projected database. For example, if we look at the frequent multidimensional sequence $S_{1}=<C_{6}$ $\mathrm{C}_{2}>$ with $\mathrm{D}_{2}=\left\{*, \mathrm{Y},{ }^{*}\right\}$ as shown in Table 4.5 , we will find that there are three records in the original datasets that have the same dimensions $\left({ }^{*}, \mathrm{Y},{ }^{*}\right)$ and two of them have the sequence $<\mathrm{C}_{6} \quad \mathrm{C}_{2}>$. Therefore we can say that the projected support is 0.667 (we write $\left.\sup _{p}\left(S_{1}, D_{2}\right)\right)=0.667$ ). This type of support is an important measure since it gives us information about how the frequent sequence was repeated within the required dimensions compared to other sequences that have the same dimensions.

These two types of supports will be used as the main parameters to determine the effectiveness of the sequence. The resources that will be reserved for each user for their future movements will be based on this effectiveness value. For each sequence $\mathrm{S}_{\mathrm{i}}=\left\langle\mathrm{s}_{1} \mathrm{~s}_{2} \ldots \mathrm{s}_{\mathrm{k}}>\right.$, we use the two supports to generate the effectiveness of using 
that sequence given the set of $n$ dimensions $D_{j}=\left\{d_{1}, d_{2}, d_{3}, \ldots, d_{n}\right\}$, and this effectiveness is donated as $E\left(S_{i} / D_{j}\right)$ which can be calculated as:

$$
E\left(S_{i} \mid D_{j}\right)=\sup _{p}\left(S_{i}, D_{j}\right) \cdot w_{1} \cdot w_{2}
$$

and

$$
\begin{gathered}
w_{1}=\left\{\begin{array}{lll}
\frac{\sup _{D B B}\left(S_{i}\right)}{\sup _{p}\left(S_{i}, D_{j}\right)} & \text { if } & \sup _{D B B}\left(S_{i}\right)>\sup _{p}\left(S_{i}, D_{j}\right) \\
1 & \text { if } & \sup _{D B}\left(S_{i}\right) \leq \sup _{p}\left(S_{i}, D_{j}\right)
\end{array}\right. \\
w_{2}=\left\{\begin{array}{lll}
1 & \text { if } & \sup _{I D B}\left(S_{i}\right) \geq \sup _{p}\left(S_{i}, D_{j}\right) \\
\frac{\sup _{p}\left(S_{i}, D_{j}\right)}{\operatorname{Max}_{\text {sup }}} & \text { if } & \sup _{D B}\left(S_{i}\right)<\sup _{p}\left(S_{i}, D_{j}\right)
\end{array}\right. \\
\operatorname{Max}_{\text {sup }}=\max _{k=1}^{N_{S}}\left(\max _{l=1}^{N_{D}}\left(\sup _{p}\left(S_{k}, D_{l}\right)\right)\right)
\end{gathered}
$$

where

$\operatorname{Max}_{\text {sup }} \quad$ is the maximum projected support found in all of the discovered sequences

$N_{D} \quad$ is the number of possible combinations between the dimensions

$N_{S} \quad$ is the total number of frequent sequences

Our model of frequent sequences is modified into the model of "effective sequences", where the effectiveness of a sequence reflects the power of that sequence in the DB compared to the other sequences. This was accomplished by considering 
the weights $w_{1}$ and $w_{2}$ shown in equation 2 . The factor $w_{1}$ was used to ensure that we consider the support in the whole database if it is larger than the support in the projected dataset. On the other hand the factor $\mathrm{w}_{2}$ was used to ensure that we consider support in the projected dataset if it is larger than the support in whole database and it also gives information about the effectiveness of this sequence compared to most frequent sequence in all projected databases.

The resources that will be reserved for each user for their future movements will be based on this effectiveness value $E\left(S_{i} / D_{j}\right)$. If there is no effect of considering the dimensions we will use the general support $\sup _{D B}\left(S_{i}\right)$ for resource reservation. Otherwise we will use the values of the projected support value which has been modified by a ratio of this support to the maximum support. This will insure that we take into consideration two the power of this sequence compared to other sequences in all projected database. The service provider will be responsible for determining the required combinations of the dimensions to be considered together. In our case, we have two dimensions $\left\{d_{1}, d_{2}\right\}$, where $d_{1}$ refers to the time interval and $d_{2}$ refers to the type of the day. We study the effect of each of these dimensions on the overall performance of the mobile networks in section 5. According to this study we can select the best combination of dimensions to be used. In reality, we need to store only the sequential patterns corresponding to this combination of dimensions and the sequential patterns that are generated without ay of these dimensions (when $D_{k}=\left\{{ }^{*}\right.$, $\left.\left.{ }^{*},{ }^{*}\right\}\right)$. These two types of sequential patterns are used in the CAC and RR algorithms as explained in the next sections. These sequential patterns are used in the CAC and RR algorithms as explained in the next chapters. 


\section{CHAPTER V}

\section{MSP-CACRR: MULTIDIMENSIONAL SEQUENTIAL PATTERNS BASED ADMISSION CONTROL FOR NEXT-GENERATION CELLULAR NETWORKS}

In this chapter, we propose a multidimensional sequential patterns (MSP) based $\mathrm{CAC}$ and $\mathrm{RR}$ technique called MSP-CACRR technique designed for next generation of cellular networks. This technique is based on the multidimensional sequence mining technique MobilePrefixSpan that has been introduced in the previous chapter. The spatial, temporal, and usage information are used in MobilePrefixSpan to extract the movement patterns of the mobile users. Comprehensive simulation was conducted to study the effect of each of the used dimensions on the network performance. Simulation results show that the proposed MSP-CACRR technique uses the sequential patterns effectively to enhance the performance of cellular networks.

\subsection{Data Collection}

The proposed MSP-CACRR scheme is mainly based on generating two types of users' mobility profiles. The first type is called the local mobility profile. This profile is generated individually for each user by his/her MH. The MH has the responsibility to collect the mobility data of its user. This data is used to build the mobility profile 
of the user and is continually updated based on its users' movements. The second type of the mobility profiles is called the global mobility profile generated by each base station (BS). The BS has the responsibility to collect the mobility data of all the users passing through the cell. This global profile will be used if the local profile gives low accuracy for future mobility prediction. The purpose of using data mining techniques is to generate mobility patterns (profiles) that can be used to predict the users' future movements. The key here is how we can effectively record and analyze the previous behavior of mobile users to generate these mobility profiles. These profiles should contain the frequent paths with the corresponding probabilities. Frequent paths will be used to predict the future movements of the MH. The CAC and RR techniques are based on the techniques that used in PCAC-RR with application of the MobilePrefixSpan to extract the mobility patterns and their supports.

\subsubsection{Data Collected by MH}

The collected data for one user will be as shown on Table 5.1. These data are collected by the mobile host (MH) of the user.

Table 5.1 Data Collected by the MH

\begin{tabular}{|c|c|c|c|c|}
\hline ID of Visited Cell & $\begin{array}{c}\text { Visit Start } \\
\text { Time }\end{array}$ & $\begin{array}{c}\text { Visit End } \\
\text { Time }\end{array}$ & $\begin{array}{c}\text { Weekend } \\
\text { Day }\end{array}$ & $\begin{array}{c}\text { Type of } \\
\text { Service }\end{array}$ \\
\hline $\mathrm{C}_{1}$ & $\mathrm{VST}_{1}$ & $\mathrm{VET}_{1}$ & $\mathrm{~W}_{1}$ & $\mathrm{ToS}_{1}$ \\
\hline $\mathrm{C}_{2}$ & $\mathrm{VST}_{2}$ & $\mathrm{VET}_{2}$ & $\mathrm{~W}_{2}$ & $\mathrm{ToS}_{2}$ \\
\hline$\ldots$ & $\ldots$ & $\ldots$ & $\ldots$ & $\ldots$ \\
\hline $\mathrm{C}_{\mathrm{n}}$ & $\mathrm{VST}_{\mathrm{n}}$ & $\mathrm{VET}_{\mathrm{n}}$ & $\mathrm{W}_{\mathrm{n}}$ & $\mathrm{ToS}_{\mathrm{n}}$ \\
\hline
\end{tabular}

where:

$\mathrm{C}_{\mathrm{i}}: \quad$ represents the ID of the current visited cell. 
$\mathrm{VST}_{\mathrm{i}}$ : represents the time stamp when this $\mathrm{MH}$ enters $\mathrm{Li}$

$\mathrm{VET}_{\mathrm{i}}$ : represents the time stamp when this MH exits Li

$\mathrm{W}_{\mathrm{i}}$ : a binary value describes if the information was recorded during the weekend or not. We will use the letter $Y$ if it was on a weekend day, otherwise we use the letter $\mathrm{N}$ (this means $\mathrm{Wi} \in\{\mathrm{Y}, \mathrm{N}\}$ ).

$\mathrm{ToS}_{\mathrm{i}}$ : represents the type of the current service provided to this $\mathrm{MH}$, where $\operatorname{ToS}_{\mathrm{i}} \in$ \{"Class-I", "Class-II", "Idle"\}

Note that the type of service can be "Idle" if the mobile phone was not used for any application at the time of record. Also, note that the day at which this service was accessed (weekend day or not) is taken as an important factor. We believe that the behavior of the users is totally changed during the weekends [48].

A new record will start if the mobile user enters a new cell, or the current type of service or the current day has been changed.

\subsubsection{Data Collected by BS}

The base station in each cell will be responsible to collect the data that represents the movements of the mobile users between this BS and other BSs. The collected data for the base station in cell $\mathrm{C}_{\mathrm{k}}$ will be as shown below in Table 5.2. BS mobility model will mainly help the mobile users to navigate smoothly from this BS to another BS in the system. 
Table 5.2 Data Collected by the BS at Cell $\mathrm{C}_{\mathrm{k}}$

\begin{tabular}{|c|c|c|c|c|c|}
\hline $\begin{array}{c}\text { Previous } \\
\text { Cell }\end{array}$ & $\begin{array}{c}\text { Visit Start } \\
\text { Time }\end{array}$ & $\begin{array}{c}\text { Next } \\
\text { Cell }\end{array}$ & $\begin{array}{c}\text { Visit End } \\
\text { Time }\end{array}$ & $\begin{array}{c}\text { Weekend } \\
\text { Day }\end{array}$ & $\begin{array}{c}\text { Type of } \\
\text { Service }\end{array}$ \\
\hline $\mathrm{CP}_{1}$ & $\mathrm{VST}_{1}$ & $\mathrm{CN}_{1}$ & $\mathrm{VET}_{1}$ & $\mathrm{~W}_{1}$ & $\mathrm{ToS}_{1}$ \\
\hline $\mathrm{CP}_{2}$ & $\mathrm{VST}_{2}$ & $\mathrm{CN}_{2}$ & $\mathrm{VET}_{2}$ & $\mathrm{~W}_{2}$ & $\mathrm{ToS}_{2}$ \\
\hline$\ldots$ & $\ldots$ & $\ldots$ & $\ldots$ & $\ldots$ & $\ldots$ \\
\hline $\mathrm{CP}_{\mathrm{n}}$ & $\mathrm{VST}_{\mathrm{n}}$ & $\mathrm{CN}_{\mathrm{n}}$ & $\mathrm{VET}_{\mathrm{n}}$ & $\mathrm{W}_{\mathrm{n}}$ & $\mathrm{ToS}_{\mathrm{n}}$ \\
\hline
\end{tabular}

where

$\mathrm{CP}_{\mathrm{i}}$ : represents the ID of the previous visited cell

$\mathrm{CN}_{\mathrm{i}}:$ represents the ID of the next visited cell and for any record in the table: $\mathrm{CP}_{\mathrm{i}}$

$\neq \mathrm{CN}_{\mathrm{i}} \neq \mathrm{C}_{\mathrm{k}}$

Once the MH and BS have collected these data, they start separately to generate the mobility paths by transforming the collected data into a sequence of symbols, where each symbol represents BS. Every path is composed of a sequence of BS IDs for each recorded visit. In addition, each path also contains the information regarding the duration of MH stay in a cell. This is achieved by collecting mobility data of a MH at fixed time slots $\Delta t$. For example if $\Delta t=2$ minutes, this means that movement data are collected every two minutes. In this case, the time between movements is included in the generated sequence of movements. This information is used to estimate the time at which the user will move from one location to another.

The mobility patterns in the BSs and MHs are generated using MobilePrefixSpan as explained in the previous chapter using the generated sequences. 


\subsection{Predictive Resource Reservation Scheme}

The RR scheme uses the frequent paths generated earlier with their corresponding probabilities to reserve the suitable bandwidth. This probability is used during the call handoff process. Each cell will have a portion of the bandwidth reserved for handoff calls. In order to make a reservation for the bandwidth, the cells have to monitor the available bandwidth at any time. For each cell $C_{i}$ the available bandwidth at any time $T_{j}$ is denoted as $B_{a}\left(C_{i}, T_{j}\right)$. The available bandwidth could be calculated if we know the following:

- $B_{t}\left(C_{i} T_{j}\right):$ the total bandwidth of cell $C_{i}$ at time $T_{j}$

- $B_{u}\left(C_{i}, T_{j}\right):$ the estimated bandwidth used by users in cell $C_{i}$ at time $T_{j}$

- $B_{r}\left(C_{i}, T_{j}\right):$ the bandwidth reserved by cell $C_{i}$ for handoff calls at time $T_{j}$

The available bandwidth in cell $C_{i}$ at any time is calculated from equation (5.1):

$$
B_{a}\left(C_{i}, T_{j}\right)=B_{t}\left(C_{i} T_{j}\right)-B_{u}\left(C_{i}, T_{j}\right)-B_{r}\left(C_{i}, T_{j}\right)
$$

The service providers determine the total bandwidth assigned to each cell during the design phase. The bandwidth used by the users in cell $C_{i}$ at the expected handoff time $T_{h}$ can be estimated easily from the bandwidth used at the current time. If there is a call, the required amounts of bandwidth to be reserved in the other cells have to be calculated to handle the handoff operation. The expected time at which the handoff will happen has to be taken into account as well. Suppose that there is a call in cell $C_{i}$ at time $T_{j}$. The proposed algorithm of the RR scheme will be executed as follows: 
1. Determine the corresponding dimensions form the current time and current day. In this case we can determine $D_{k}=\left\{d_{1}, d_{2}, d_{3}\right\}$ where $d_{1}$ refers to the time interval, $d_{2}$ refers to the type of the day, and $d_{3}$ refers to the type of service.

2. According to the set of dimensions $D_{k}$, start to look at the sequential patterns stored in the $\mathrm{MH}$ of the current user that are corresponding to these dimensions to predict the frequent sequences.

3. If all of the current dimensions can not be supported by the stored sequential patterns, we use $\mathrm{D}_{\mathrm{k}}=\{*, *, *\}$ to predict the frequent sequences..

4. If there is at least one predicted frequent sequence, then the bandwidth reservation is performed based on sequential patterns stored in the $\mathrm{MH}$ as follows:

i. For each cell $C_{k}$ in the predict sequence $\left(\mathrm{S}_{1}\right)$, estimate the handoff time $\left(T_{h}\right)$ using the known time slot $\Delta t$.

ii. Once we find $T_{h}$, check the available bandwidth at $T_{h}$. The available bandwidth could be calculated from equation (5.1).

iii. The required amount of bandwidth $B_{\text {req }}$ to be reserved is determined as a portion from the actual required bandwidth $B_{\text {actual according to the }}$ effectiveness of the path using the following equation:

$$
B_{\text {req }}=B_{\text {actual }} * E\left(\mathrm{~S}_{1}, \mathrm{D}_{\mathrm{k}}\right)
$$


iv. If there is enough bandwidth available at time $T_{h}$, then the amount of bandwidth to be reserved will be $B_{\text {req }}$ and then the available and the reserved bandwidths, are updated.

v. If there is not sufficient bandwidth available and the call is from Class-I, then this call can borrow some amount $\left(B_{\text {borrow }}\right)$ form the Class-II calls in that cells.

vi. Otherwise the amount of bandwidth to be reserved will be the available bandwidth plus $B_{\text {borrow }}$

5. If no frequent sequence could be predicted using sequential patterns stored in the $\mathrm{MH}$, then we have to access the sequential patterns stored in the $\mathrm{BS}$, and use the frequent sequences in a way similar to the one described in the step 2 to reserve the required bandwidth.

\subsection{Predictive Call Admission Control Scheme}

The admitted call may be a new call or a handoff call. Each of these categories of calls will be handled in a different way taking into account that the handoff calls will have a priority over the new calls. If the admission is performed at the cell $C_{i}$ and at the time interval $T_{j}$, then the CAC algorithm can be given as follows:

a. For the new calls from Class-I:

i. According to the set of dimensions $D_{k}$, start to look at the sequential patterns stored in the $\mathrm{MH}$ of the current user that are corresponding to these dimensions to predict the frequent sequences. 
ii. If all of the current dimensions can not be supported by the stored sequential patterns, we use $D_{k}=\{*, * *\}$ to predict the frequent sequences. If no frequent sequence could be predicted using sequential patterns stored in the $\mathrm{MH}$, then we have to access the sequential patterns stored in the BS

iii. According to $\mathrm{D}_{\mathrm{k}}$ and the current cell $\mathrm{C}_{\mathrm{i}}$, check the available $\mathrm{BW}$ in the current cell $\mathrm{C}_{\mathrm{i}}$ (which is given by $B_{a}\left(C_{i}, T_{j}\right)$ ) as well as $\mathrm{BW}$ in the next expected cells in the predicted frequent sequence with high probabilities above a predetermined threshold $L_{p}$. In our case we used $L_{p}=0.8$.

iv. If each of the next expected cells has available BW to cover this new call at the next time step, then the call is accepted and the required bandwidth is allocated.

$\mathrm{v}$. Otherwise the call is blocked.

b. For the new calls from Class-II:

i. Check only the available BW at the current cell $\mathrm{C}_{\mathrm{i}}$, at which the admission is performed.

ii. If the required $\mathrm{BW}$ is less than or equal the $B_{a}\left(C_{i}, T_{j}\right)$, then the call is accepted and the required $\mathrm{BW}$ is allocated.

iii. If the required $\mathrm{BW}$ is greater than $B_{a}\left(C_{i}, T_{j}\right)$, and there is any available $\mathrm{BW}$ $\left(B_{a}\left(C_{i}, T_{j}\right) \neq 0\right)$, then the call is accepted and the available $\mathrm{BW}$ is allocated. Then, this bandwidth readjusted in future when there is BW available.

iv. Otherwise the call is blocked.

c. For the handoff calls from Class-I: 
i. Handoff calls can allocate bandwidth not only from the reserved bandwidth, but also from the available bandwidth (while the new calls can allocate bandwidth from the available bandwidth only).

ii. Check the bandwidth reserved for the handoff calls added to the available bandwidth in the cells at that time interval $\left(B_{r}\left(C_{i}, T_{j}\right)+B_{a}\left(C_{i}, T_{j}\right)\right)$.

iii. If the required bandwidth $\leq\left[B_{r}\left(C_{i}, T_{j}\right)+B_{a}\left(C_{i}, T_{j}\right)\right]$., then the call is accepted and the bandwidth is allocated according to the required bandwidth.

iv. Otherwise the call is dropped.

d. For the handoff calls from Class-II:

i. The call will be accepted if there is bandwidth available in the reserved or the available bandwidths.

ii. If the required $\mathrm{BW}$ is less than or equal the $\left[B_{r}\left(C_{i}, T_{j}\right)+B_{a}\left(C_{i}, T_{j}\right)\right]$, then the call is accepted and the required BW is allocated.

iii. If the required $\mathrm{BW}$ is greater than $\left[B_{r}\left(C_{i}, T_{j}\right)+B_{a}\left(C_{i}, T_{j}\right)\right]$, and $\left[B_{r}\left(C_{i}, T_{j}\right)+B_{a}\left(C_{i}\right.\right.$ ,$\left.\left.T_{j}\right)\right] \neq 0$, then the call is accepted and the allocated BW will be the minimum of $B_{r}\left(C_{i}, T_{j}\right)$ and $B_{a}\left(C_{i}, T_{j}\right)$. Then, this bandwidth readjusted in future when there is BW available.

iv. Otherwise the call is blocked.

This approach will maximize the probability of completing the call without dropping during the user navigation through the cells. In other words, this approach 
will prevent us from accepting a new calls that have high probability of dropping. Moreover, this approach performs the best effort that enables the network to accept as many calls as possible. 


\section{CHAPTER VI}

\section{PAC-WHN: PREDICTIVE ADMISSION CONTROL FOR WIRELESS HETEROGENEOUS NETWORKS}

It is expected that the fourth generation $(4 \mathrm{G})$ mobile networks will support more multimedia communications and provide mobile services every time and everywhere. Mobile communications will take a central role in our daily lives and this role will expand as our lifestyles use mobile communications as a stepping stone to improve the quality of life over the next ten years [49]. One way to ensure that users of the future mobile system do not have restricted service options is to ensure that new terminals can handle the existing system as well as the new one. Furthermore, future mobile communication networks will be integrated with heterogeneous access methods and various kinds of cells. This can be accomplished by integrating several radio access networks (RANs) (like cellular networks (2G, 3G, etc.), Wireless LAN (WLAN) and Mobile Ad hoc Networks (MANET)) in developing what is called "wireless heterogeneous networks" (WHN). Accordingly, effective connection management and handover between heterogeneous systems are required. In this chapter, we introduce a predictive $\mathrm{CAC}$ and RR technique called $P C A-W H N$ that can support the $4 \mathrm{G}$ wireless heterogeneous networks (WHN). This new technique is based on the multidimensional sequence mining technique MobilePrefixSpan that has been introduced in chapter 4 . 


\subsection{Introduction}

The mobile users in the suggested wireless heterogeneous networks can communicate in different ways according to many positioning and networking factors. Admission control of the new calls and the handoff calls is more complex in WHN because different types of radio access networks (RANs) have different interfaces and different capabilities [49-60]. Choosing a suitable RAN to be connected to and maintaining the communication while the user is moving inside the RAN and between different RANs is one of the important and hot research problems for the next generation of mobile networks. All the existing admission control schemes proposed by researchers in this area deal with a single RAN and none of these schemes was designed for multiple RANs as in the WHN.

PCA-WHN is a predictive CAC and RR technique that uses an efficient multidimensional sequence mining technique to extract the mobility profiles (models) of the mobile users. PCA-WHN takes into account the different characteristics of the cellular networks and the WLANs and it is based on using the spatial information, the temporal information and the usage information of the mobile users. We used MobilePrefixSpan technique in PCA-WHN to generate the mobility patterns of the mobile users. MobilePrefixSpan can work in the mobile environment and uses the collected information to generate the mobility patterns as explained in chapter 4 .

In the next section, we explain the architecture of the communication systems supported by PCA-WHN. Section three gives the details of the collected data. The details of the proposed PCA-WHN technique are explained in the last two sections. 


\subsection{Architecture of $4 \mathrm{G}$ Mobile Communication Systems Supported by PAC-WHN}

The architecture of the proposed heterogeneous wireless networks includes WLANs and cellular networks and assumes an All-IP based structure as proposed in the literature [49-63]. All-IP wireless and mobile networks represent the convergence of two key technologies: Internet and wireless cellular systems [61]. The core IP network will serve as the backbone network with internet connectivity and packet data services $[49,50,55]$.

The architecture of the proposed wireless heterogeneous network is shown in Fig. 6.1. As we can see, there are four basic components of the proposed architecture: 1) Base Stations, 2) Access Points, 3) Mobile Hosts, and 4) IP Core Network. The WLAN access points (APs) represent the fixed communication points for the WLAN while the base stations (BSs) represent the fixed communication points for the cellular networks. Mobile hosts (MHs) are supposed to be designed to work in two different modes: dual-ode and single-mode. Dual-mode mobile hosts will be able to support services provided by WLANs and cellular networks while singlemode mobile hosts will support only one type of mobile technology. The proposed WHN will be able to support dual mode mobile hosts as will as single-mode mobile hosts.

By design, cellular networks are aimed at users with high mobility and low connection rates while WLAN networks are aimed at users with low mobility and high connection rates $[49,50,57,64,65]$. Also, the cost of using the cellular 
networks is higher than the cost of using WLAN. Table 6.1 [57] summarizes the characteristics of the BS in cellular networks and the AP in the WLAN.

It is important in wireless heterogeneous networks to distinguish between the different types of the services that can be provided by the networks. In general, we can classify the services into two main categories according to their delay sensitivity characteristics. One is real-time services (Class I), that are sensitive to delay, such as the conversation and streaming applications. The other includes non-real time services that are insensitive to delay (Class II); such as interactive and background applications. The detailed classification of the provided services is shown Table 6.2 [57]. We believe that this classification is very important for most of the protocols and algorithms designed for future wireless heterogeneous networks. This is due to the nature of the WHN where we have more than one type of networks (RANs) with different capabilities. In this case, the goal will be to connect each of the mobile users to one of the available RANs that will be able to provide the required service with high efficiency.

Table 6.1 AP and BS Characteristics

\begin{tabular}{|c|c|c|c|c|}
\hline RAN & Coverage & Data Rate & Cost & Typical User \\
\hline AP & Limited & $1 \sim 11 \mathrm{Mbps}$ & Low & Low Mobility \\
\hline BS & Unlimited & $9.6 \sim 300 \mathrm{Kbps}$ & High & Fast Mobility \\
\hline
\end{tabular}



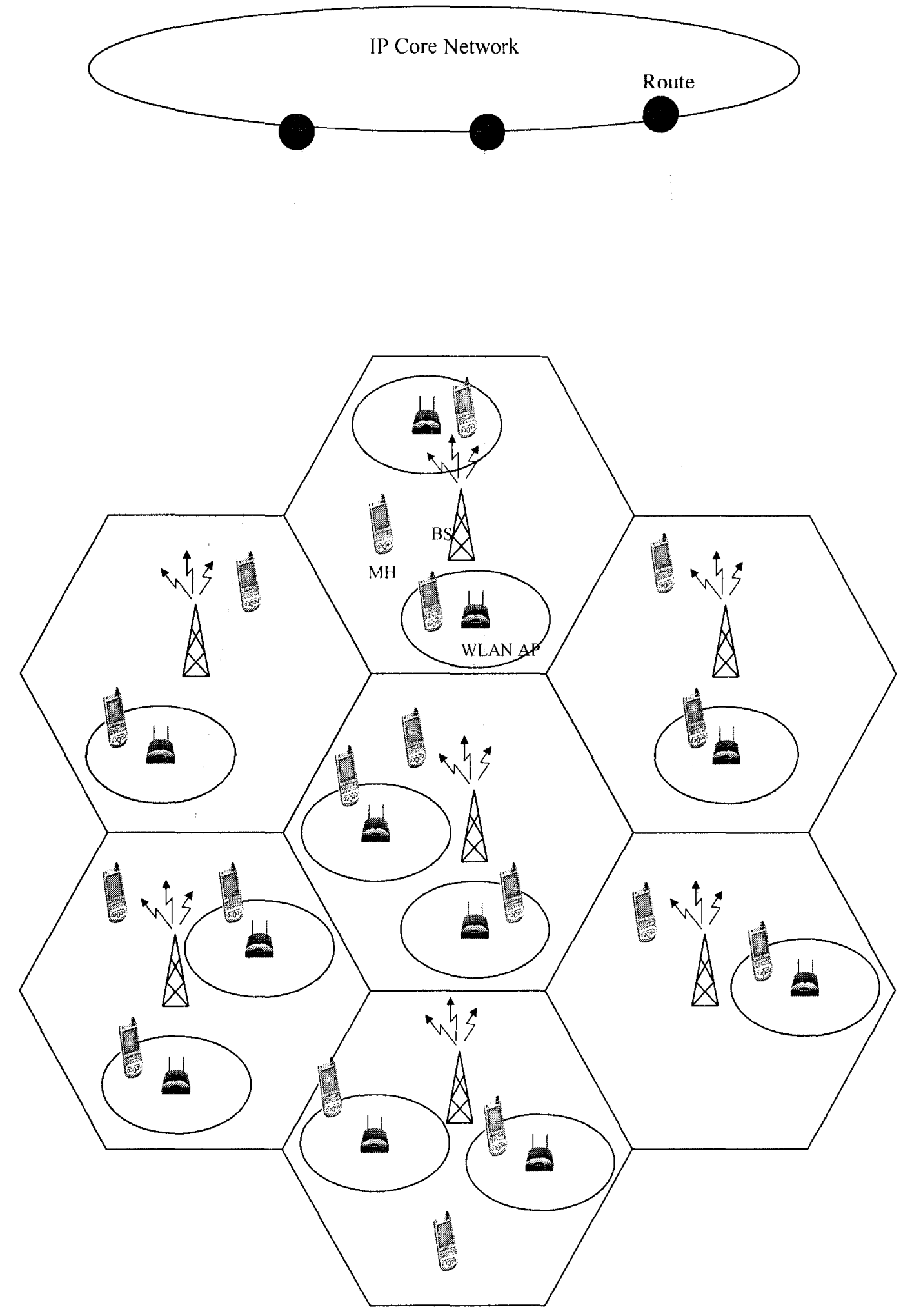

Fig. 6.1 Architecture of Proposed Wireless Heterogeneous Networks 
Table 6.2 Traffic Class for 4G Wireless Heterogeneous Networks [57]

\begin{tabular}{|l|c|c|c|c|}
\hline \multirow{2}{*}{} & \multicolumn{2}{|c|}{ CLASS I } & \multicolumn{2}{c|}{ CLASS II } \\
\cline { 2 - 5 } & $\begin{array}{c}\text { Conversational } \\
\text { Applications }\end{array}$ & $\begin{array}{c}\text { Streaming } \\
\text { Applications }\end{array}$ & $\begin{array}{c}\text { Interactive } \\
\text { Applications }\end{array}$ & $\begin{array}{c}\text { Background } \\
\text { Applications }\end{array}$ \\
\hline BER & $10^{-3}$ & $10^{-5}$ & $10^{-8}$ & $10^{-9}$ \\
\hline Delay & Strict and low & Bounded & Tolerable & Unbounded \\
\hline $\begin{array}{l}\text { Guaranteed } \\
\text { Rate }\end{array}$ & Yes & Yes & No & No \\
\hline Application & $\begin{array}{c}\text { Voice, Internet } \\
\text { Game }\end{array}$ & $\begin{array}{c}\text { VOD, Cable } \\
\text { TV }\end{array}$ & Web, Telnet & FTP. E-mail \\
\hline \multicolumn{2}{|r|}{ Real-time service } & \multicolumn{2}{c|}{ Non-real time service } \\
\hline
\end{tabular}

The configuration of the wireless heterogeneous networks will be based on the connectivity to IP networks [49-60]. The advantages of using IP as a core network protocol are $[49,55]$ :

- Internet connectivity

- Efficient transmission of IP packets,

- Co-existence with other access systems

- Ease of system introduction and expandability.

- IP networks can also connect with or accommodate wireless access systems other than $4 \mathrm{G}$ systems.

- Providing better security requirements.

The MH will be able to communicate with one BS/AP according to the coverage area and its mode of communications. The connection can be handed off from one AP to another AP (or from BS to another BS), and this called horizontal handoff. The connection can also be handed off form WLAN to cellular networks or vise versa and this is called vertical handoff. In our system model, we can have 
several WLANs inside the same cell but they do not have overlapped coverage. So the mobile user at every moment has the following possibilities of coverage:

- Covered by cellular network only

- Covered by cellular network and WLAN.

Also, we assume that each of the hotspots (AP) will cover a part of a single cell and can not cover any other cell. We used these assumptions to reduce the complexity of the system.

In our system model, we assume that we can have several WLAN inside the same cell but they are not intersected. So the mobile user at every moment has the following possibilities of coverage:

- Covered by cellular network only

- Covered by cellular network and WLAN.

Also, we assume that the WLAN covers the hotspots inside a single cell and can't cover any area from another cell at the same time. We used these assumptions (restrictions) to reduce the complexity of the system.

\subsection{Data Collection}

The proposed PCA-WHN technique is mainly based on generating two types of users' mobility profiles using MobilePrefixSpan technique. The first type is called the local mobility profile. This profile is generated individually for each user by his/her MH. The MH will have the responsibility to collect the mobility data of its user. This data is used to build the mobility profile of the user and is continually updated based on its users' movements. The second type of the mobility profiles is called the global 
mobility profile generated by each $\mathrm{BS}$ and $\mathrm{AP}$. The BS/AP has the responsibility to collect the mobility data of all the users passing through the cell. This global profile will be used if the local profile gives low accuracy for future mobility prediction. The purpose of using data mining techniques is to generate mobility patterns (profiles) that can be used to predict the users' future movements. In this section we will explain how the data are collected in the MH and the BS/AP.

\subsubsection{Data Collected by Mobile Host}

The data collected by the MH is used to build the local mobility model that can describe the behavior of mobile users for a period of time. The data items collected for this model are shown in Table 6.3, where:

$L_{i}: \quad$ represents the ID of the current visited cell or current access point for the record number $i$ in data collected table.

$V S T_{i}: \quad$ represents the time stamp when this $\mathrm{MH}$ enters $\mathrm{L}_{\mathrm{i}}$

$V E T_{i}$ : represents the time stamp when this $\mathrm{MH}$ exits $\mathrm{L}_{\mathrm{i}}$

$W_{i}$ : a binary value describes if the information was recorded during the weekend or not. We will use the letter $Y$ if it was on a weekend day, otherwise we use the letter $\mathrm{N}$ (this means $\mathrm{Wi} \in\{\mathrm{Y}, \mathrm{N}\}$ ).

$T o S_{i}$ : represents the type of the current service provided to this $\mathrm{MH}$, where $\operatorname{ToS}_{\mathrm{i}} \in\{$ "Class-I", "Class-II", "Idle"\} 
Table 6.3 Data Collected by the $\mathrm{MH}$

\begin{tabular}{|c|c|c|c|c|}
\hline $\begin{array}{c}\text { ID of Visited } \\
\text { BS/AP }\end{array}$ & $\begin{array}{c}\text { Visit Start } \\
\text { Time }\end{array}$ & $\begin{array}{c}\text { Visit End } \\
\text { Time }\end{array}$ & $\begin{array}{c}\text { Is this on a } \\
\text { Weekend Day ? }\end{array}$ & $\begin{array}{c}\text { Type of } \\
\text { Service }\end{array}$ \\
\hline $\mathrm{L}_{1}$ & $\mathrm{VST}_{1}$ & $\mathrm{VET}_{1}$ & $\mathrm{~W}_{1}$ & ToS $_{\mathrm{I}}$ \\
\hline $\mathrm{L}_{2}$ & $\mathrm{VST}_{2}$ & $\mathrm{VET}_{2}$ & $\mathrm{~W}_{2}$ & $\mathrm{ToS}_{2}$ \\
\hline$\ldots$ & $\ldots$ & $\ldots$ & $\ldots$ & $\ldots$ \\
\hline $\mathrm{L}_{\mathrm{n}}$ & $\mathrm{VST}_{\mathrm{n}}$ & $\mathrm{VET}_{\mathrm{n}}$ & $\mathrm{W}_{\mathrm{n}}$ & $\mathrm{ToS}_{\mathrm{n}}$ \\
\hline
\end{tabular}

Note that a new record will start if the mobile user enters a new BS/AP or if the current type of service is changed. The type of service can be "idle" if the mobile phone was not communicating at the time of data recording. Also, note that the day at which this service was accessed (weekend or not) is included as an important factor. We believe that the behavior of the users is totally different during the weekends.

To generate the mobility paths, it is necessary to transform the collected data from Table 3 at the MH into a sequence of symbols, where each symbol represents BS/AP. Every path is composed of a sequence of BS/AP IDs for each recorded visit. In addition, each path also contains the information regarding the duration of MH stay in a cell. This is achieved by collecting mobility data of a $\mathrm{MH}$ at fixed time slots $\Delta t$. For example if $\Delta t=2$ minutes, this means that movements data are collected every tow minutes. In this case, the time between movements is included in the generated sequence of movements. This information is used to estimate the time at which the user will move from one location to another.

Any generated path $P_{M H}$ can be represented as:

$$
P_{M H}=<L_{1} \quad L_{2} \quad \ldots L_{n}>
$$

where

$$
\begin{aligned}
& L_{i} \in\{B \cup A\} \\
& B=\left\{C_{1}, C_{2}, \ldots, C_{m}\right\} \quad \text { and } A=\left\{P_{1}, P_{2}, \ldots, P_{k}\right\}
\end{aligned}
$$


and

$C_{i}: \quad$ is a cell ID.

$B: \quad$ is the set of all cells IDs in the system.

$P_{i}: \quad$ is an access point ID.

$A: \quad$ is the set of all access points IDs in the system.

\subsubsection{Data Collected by Base Station and Access Point}

The base station in each cell will also be responsible to collect the data that represent the movements of the mobile users between this BS and other BSs or APs. The format of collected data for the base station in cell $C_{i}$ is shown in Table 6.4, where:

$L P_{i}$ : represents the ID of the previous visited cell or access point and $L P_{i} \in\{B \cup$ A\}.

$L N_{i}$ : represents the ID of the next visited cell or access point and $L N_{i} \in\{B \cup A\}$ and for any record in the table: $\quad \mathrm{LP}_{\mathrm{i}} \neq \mathrm{LN}_{\mathrm{i}} \neq \mathrm{C}_{\mathrm{i}}$

Table 6.4 Data Collected by the $\mathrm{BS}$ at $\mathrm{C}_{1}$

\begin{tabular}{|c|c|c|c|c|c|c|}
\hline $\begin{array}{c}\text { Current } \\
\text { BS }\end{array}$ & $\begin{array}{c}\text { Previo } \\
\text { us } \\
\text { BS/AP }\end{array}$ & $\begin{array}{c}\text { Visit Start } \\
\text { Time }\end{array}$ & $\begin{array}{c}\text { Next } \\
\text { BS/AP }\end{array}$ & $\begin{array}{c}\text { Visit End } \\
\text { Time }\end{array}$ & $\begin{array}{c}\text { Is this on a } \\
\text { Weekend } \\
\text { Day? }\end{array}$ & $\begin{array}{c}\text { Type of } \\
\text { Service }\end{array}$ \\
\hline $\mathrm{C}_{\mathrm{i}}$ & $\mathrm{LP}_{1}$ & $\mathrm{VST}_{1}$ & $\mathrm{LN}_{1}$ & $\mathrm{VET}_{1}$ & $\mathrm{~W}_{1}$ & $\mathrm{ToS}_{1,}$ \\
\hline $\mathrm{C}_{\mathrm{i}}$ & $\mathrm{LP}_{2}$ & $\mathrm{VST}_{2}$ & $\mathrm{LN}_{2}$ & $\mathrm{VET}_{2}$ & $\mathrm{~W}_{2}$ & $\mathrm{ToS}_{2}$ \\
\hline$\ldots$ & $\ldots$ & $\ldots$ & $\ldots$ & $\ldots$ & $\ldots$ & $\ldots$ \\
\hline $\mathrm{C}_{\mathrm{i}}$ & $\mathrm{LP}_{\mathrm{n}}$ & $\mathrm{VST}_{\mathrm{n}}$ & $\mathrm{LN}_{\mathrm{n}}$ & $\mathrm{VET}_{\mathrm{n}}$ & $\mathrm{W}_{\mathrm{n}}$ & $\mathrm{ToS}_{\mathrm{n}}$ \\
\hline
\end{tabular}


Once the BS/AP has collected these data components, it can start the process of analyzing these data with the objective of building the BS Mobility Model which describes the expected movements of the users who passes through this BS. This profile is based on the type of services as well as period of time. This model will be based on the following main parameters:

- The type of the service.

- The day at which this service was accessed (weekend or not): we believe that the behavior of the users is totally changed during the weekends.

- The time at which this service was provided.

BS/AP mobility model will be used if the MH mobility model is not able to predict the future path of the mobile users. For example, if a mobile user enters a new location that has not been visited before. In this case the mobility models of BS/AP that covers this location will be used to predict the future path.

Any path $P_{B S}$ in the BS mobility model will be of length three because it represents the previous, current, and next BSs/APs. We used only the length of three for each path in the BS/AP because using longer length will result in more signaling overhead to transfer the location information between the BSs and the APs. Each path in the BS/AP can be represented as:

$$
P_{B S}=<L_{1} \quad L_{2} \quad L_{3}>
$$

where

$$
\begin{aligned}
& L_{1} \in\{B \cup A\} \text { and } \\
& L_{2} \in B \\
& L_{3} \in\{B \cup A\} \\
& L_{1} \neq L_{2} \neq L_{3}
\end{aligned}
$$




$$
B=\left\{C_{l}, C_{2}, \ldots, C_{m}\right\} \quad \text { and } \quad A=\left\{P_{l}, P_{2}, \ldots, P_{k}\right\}
$$

and

$C_{i}: \quad$ is a cell ID.

$B: \quad$ is the set of all cells IDs in the system.

$P_{i}: \quad$ is an access point ID.

$A$ : is the set of all access points IDs in the system.

The access point in each WLANs will also be responsible for collecting similar type of information about the users passing through it. Here we focused in explanation on the BS, the similar approach is also applied to the AP.

\subsection{Resource Reservation in PCA-WHN}

The proposed resource reservation technique is based mainly on the fact that we have different classes of mobile services (as shown in Table 6.2). The services CLASS I (real time services) will need a guaranteed transitions rate in order to provide the required services. In order to ensure the continuity of the services of CLASS I without any interruption in the service, while the users are moving form BS/AP to another BS/AP (vertical or horizontal handoffs), we need to reserve the required resources for these users in the expected locations that will be visited by the mobile users. On the other hand, for services from CLASS II (non-real time services), we may reserve resources as they become available, because CLASS II does not support guranted transmission rate. 
Each BS/AP will have a portion of the bandwidth reserved for handoff calls. In PCA-WHN technique, we divide available bandwidth into two sub-portions:

a) Reserved for handoff calls form CLASS I.

b) Reserved for handoff calls form CLASS II.

In order to make a reservation for the bandwidth, each BS/AP has to monitor the available bandwidth at all times. For each location $L_{i}(\mathrm{BS} / \mathrm{AP})$ the available resources (bandwidth) at any time $T_{j}$ is denoted as $R_{a}\left(L_{l}, T_{j}\right)$. The available resources could be calculated if we know the following:

$R_{t}\left(L_{i}, T_{j}\right):$ the total resources of location $L_{i}$ at time $T_{j}$

$R_{u}\left(L_{i}, T_{j}\right):$ the estimated resources used by users at location $L_{i}$ at time $T_{j}$

$R_{h l}\left(L_{i}, T_{j}\right):$ the resources reserved by location $L_{i}$ for CLASS I handoff calls at time $T_{j}$ $R_{h 2}\left(L_{i}, T_{j}\right):$ the resources reserved by location $L_{i}$ for CLASS II handoff calls at time $T_{j}$

The available resources at location $L_{i}$ at any time is calculated from the following equation:

$$
R_{a}\left(L_{i}, T_{j}\right)=R_{t}\left(L_{i}, T_{j}\right)-R_{u}\left(L_{i}, T_{j}\right)-R_{h l}\left(L_{i}, T_{j}\right)-R_{h 2}\left(L_{i}, T_{j}\right)
$$

Fig. 6.2 shows how these resources (bandwidth) can be used for different types of services. Note that $R_{h 2}$ can be used to handle handoff calls from CLASS I or CLASS II while $\mathrm{R}_{\mathrm{h} 1}$ can only used to reserve bandwidth for CLASS I handoff calls. This gives CLASS I calls a priority over the CLASS II calls. 
We will need to reserve resources for handoff calls to ensure the continuity of the call. This means if we accept the calls, then the resources in the future locations (AP/BS) will be reserved based on the predicted path. In WHN, we have mainly two types of handoffs:

- Horizontal handoff: this is performed between two locations (BSs/APs) inside the same RAN (for example between the BSs in the cellular networks).

- Vertical handoff: this is performed between two locations (BSs/APs) from different RANs (for example moving from cellular network to WLAN).

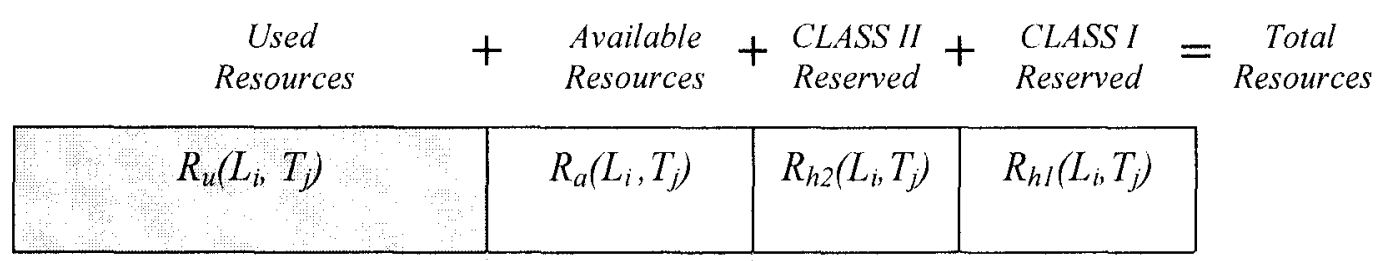

Can be used for CLASS I handoff calls

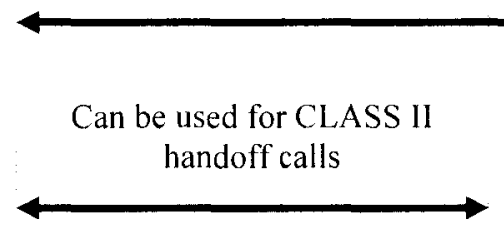

Can be used for new calls

Fig. 6.2 Distribution of the total resources in BS/AP and how to use these resources 
The main trigger for handoff in cellular networks is a weak signal received by the mobile host $(\mathrm{MH})$ from the current BS (for example weak signal received from the current BS in the cellular network). In the new WHN where we have the cellular networks and the WLAN there are many reasons to perform handoff $[55,66]$ :

i. Weak signal received from current AP (as before)

ii. The RAN where the current BS/AP connected to it can't support the current type of service with the required QoS (the call is changing from voice to media).

iii. The load is increasing in the current RAN and there is another RAN that is available.

iv. The QoS provided by one interface improves or degrades (coverage, data rate, power consumption, etc).

v. An interface currently used fails, so the MS cannot receive its flows anymore.

vi. A new interface is available, e.g., the MS enters the coverage area of a new access network.

Using the PCA-WHN technique, the decision can be taken by the AP/BS or by the MH:

- The MH of the mobile user will be able to predict the handoff times, the next path, and the expected type of services at that handoff. This is done by determining the corresponding dimensions form the current time, current day, or the type of the current call. In this case we can determine $D_{k}=\left\{d_{1}, d_{2}\right.$, 
$\left.d_{3}\right\}$ where $d_{1}$ refers to the time interval, $d_{2}$ refers to the type of the day, and $d_{3}$ refers to the type of service.

- According to the set of dimensions $\mathrm{D}_{\mathrm{k}}$, start to look at the sequential patterns stored in the $\mathrm{MH}$ of the current user that are corresponding to $\mathrm{D}_{\mathrm{k}}$ to predict the frequent sequences.

- If there is at least one predicted frequent sequence, then the bandwidth reservation is performed based on sequential patterns stored in the $\mathrm{MH}$ :

vii. For each cell $B S / A P$ in the predict sequence $\left(\mathrm{S}_{1}\right)$, estimate the handoff time $\left(T_{h}\right)$ using the known time slot $\Delta t$.

viii. Once we find $T_{h}$, check the available bandwidth at $T_{h}$. The available bandwidth could be calculated from equation (6.1).

ix. The required amount of bandwidth $R_{\text {req }}$ to be reserved is determined as a portion from the actual required bandwidth $R_{\text {actual }}$ according to the effectiveness of the sequence (which is calculated using the MobilePRefixSpn technique as explained in chapter 4) using the following equation:

$$
R_{\text {req }}=R_{\text {actual }} * E\left(\mathrm{~S}_{1}, \mathrm{D}_{\mathrm{k}}\right)
$$

- Based on this information, the handoff predicted by the MH may be for another AP/BS in the same RAN or in other RAN (horizontal or vertical handoff). Here the handoff (horizontal or vertical) is based on the mobility models generated by the MH (local mobility models).

- If no frequent sequence could be predicted using sequential patterns stored in the $\mathrm{MH}$, then the sequential patterns stored in the BS/AP have to be accessed, 
and use the frequent sequences in a way similar to the one described in the step 2 to reserve the required bandwidth.

- According to Fig. 6.2, $R_{h 2}+R_{h 1}+R_{a}$ are used to reserve resources for handoff calls from CLASS I. and $R_{h 2}+R_{a}$ are used to reserve resources for handoff calls from CLASS II

- The AP/BS monitors its load over the network and it can force some of the users to handoff to other $\mathrm{AP} / \mathrm{BS}$ if the load is expected to increase dramatically.

- The available BW should be updated after each reservation.

\subsection{Admission Control in PCA-WHN}

The admission control (AC) can be performed for the new calls and the handoff calls. The $\mathrm{AC}$ of the new calls is based on mobility models generated by the MH that requested the new call. The reason is that based on these models, the $\mathrm{MH}$ will be able to predict the next possible movements for its user according to previous behavior of this user. We define the following rules for the new calls:

- If the new call is from CLASS I, we can check the resources in the current $\mathrm{BS} / \mathrm{AP}$ by checking $R_{a}+R_{h 2}$ according to the time of service or the expected time of service.

- If the new call is from CLASS II, we can check the resources in the current BS/AP by checking only $R_{a}$ 
The detailed algorithm for admission control is shown in Appendix D. Every new call, will satisfy one of the following two cases according to the type of the services and the current RAN where the MH is connected to. In the first case, the requested service by this new call is better to be supported by another RAN. For example, if a mobile user is currently connected to the WLAN (the current RAN) and he/she wants to initiate a new voice call. In this case, it is better to use the cellular networks (the other RAN) to support the new call instead of using the WLAN. In this case, the algorithm will start to initiate a vertical handoff between from one RAN to another RAN if there is available bandwidth to the support the service. This is accomplished by predicting the expected path (according to the mobility models), and check the available resources. Otherwise, the call will be supported by the current RAN or rejected (if there are no available resources). In the second case, the service requested by the new call is better to be supported by the current RAN. For cxample, if a mobile user is currently connected to the cellular networks and he/she wants to initiate a new voice call. In this case, the algorithm will start to predict the expected path and check the available resources. The algorithm will initiate a vertical handoff if there is no available bandwidth to support the call by the current RAN. The call will be rejected if it can not be supported by any of the available RANs.

For handoff calls, we are interested in determining if the AP/BS will be able to accept and allocate resources for the admitted handoff call. This could be processed by vertical or horizontal handoff. For CLASS I handoff calls, the available resources and the reserved resources for both classes $\left(R_{a}+R_{h 2}+R_{h 1}\right)$ in the BS/AP are checked according to the time of service or the expected time of service. If the handoff call is 
from CLASS II we can check the resources in the BS/AP by checking the available resources and the reserved resources for CLASS II $\left(\mathrm{R}_{\mathrm{a}}+\mathrm{R}_{\mathrm{h} 2}\right)$. The handoff call will be accepted if there resources are available. Otherwise the call will be dropped. 


\section{CHAPTER VII}

\section{SIMULATION AND EXPERIMENTAL RESULTS}

\subsection{Simulation Model}

In order to evaluate the performance of the proposed $\mathrm{CAC}$ and $\mathrm{RR}$ techniques using different type of mobility profiles, we have built a simulation tool using the $\mathrm{C}$ language to study the performance of the proposed scheme and to compare these new techniques with other previous schemes. The simulation parameters are as shown in Table 7.1. The call arrival rate is assumed to follow a Poisson distribution with mean $\lambda$ (calls/second) in each cell (from 0.01 to 0.1 ). The call duration (call service time) is assumed to be exponentially distributed with a mean of 180 seconds.

The simulation starts by building the topology of the cells. Some of these cells are marked as used cells and the others are marked as unused cells. The unused cells simulate the natural structure of the roads where there are some constraints to place base stations in some places (like river, mountain, etc...). The used cells are then divided into home cells, work cells or ordinary cells [67]. These cells will be associated for the users to travel through according to the moving statistics shown in Table 7.2 [68]. These statistics show the main trips for each user, the percentage of each trip, and the average distance of the trip. The users' movements in our simulation are based on these statistics. The "vacation trips" defined in [68] are 
omitted in our simulation because this type of trips has a very low percentage of occurrence. The choice of home cells and work cells for each user is also based on these statistics. The topology model generated by the simulation is shown in Fig. 7.1 with all roads, home cells, work cells, unused cells and ordinary cells.

The simulation has two phases. The first phase is the initialization phase. The main objective during this phase is to collect the movement data, which will be used to generate the local and global profiles. The simulation parameters and statistics, described before, are used to generate the movements of the users between the different cells. In this initial period of simulation, we focus only on collecting the data of movements between the cells. Hence a simple CAC technique based on the availability of the resources at the time of request is used in the initial period. Also during this period, a fixed bandwidth is reserved for handoff calls.

Table 7.1 Simulation parameters

\begin{tabular}{|l|l|}
\hline \multicolumn{1}{|c|}{ Parameter } & \multicolumn{1}{c|}{ Value } \\
\hline Number of cells & 128 cells \\
\hline Type of cells & Hexagonal cells \\
\hline Diameter of each cell & $1 \mathrm{mi}$ \\
\hline Call arrival rate & $\begin{array}{l}\text { Poisson distribution with mean } \lambda \text { (varies from } 0.01 \text { to } 0.1 \\
\text { calls/second) }\end{array}$ \\
\hline Call duration time & Exponentially distribution with mean $\mu=180$ seconds \\
\hline Maximum Speed & $60 \mathrm{mi} / \mathrm{h}$ \\
\hline $\begin{array}{l}\text { Channel capacity of each } \\
\text { cell }\end{array}$ & 40 bandwidth units (BU) \\
\hline $\begin{array}{l}\text { BW required by a voice } \\
\text { call }\end{array}$ & $1 \mathrm{BU}$ \\
\hline $\begin{array}{l}\text { BW required by a text } \\
\text { call }\end{array}$ & $2 \mathrm{BUs}$ \\
\hline $\begin{array}{l}\text { BW required by a audio } \\
\text { call }\end{array}$ & $5 \mathrm{BUs}$ \\
\hline $\begin{array}{l}\text { BW required by a video } \\
\text { call }\end{array}$ & $10 \mathrm{BUs}$ \\
\hline
\end{tabular}


Table 7.2 Moving statistics [68]

\begin{tabular}{|l|c|c|}
\hline \multicolumn{1}{|c|}{ Purpose of Trip } & Percentages of Trip & $\begin{array}{c}\text { Average Trip Distance } \\
\text { (mi) }\end{array}$ \\
\hline To/From Work & $20.2 \%$ & 10.65 \\
\hline $\begin{array}{l}\text { Work-Related } \\
\text { Business }\end{array}$ & $1.4 \%$ & 28.20 \\
\hline Personal Business & $52.9 \%$ & 6.74 \\
\hline Social/Recreation & $25.3 \%$ & 11.53 \\
\hline Vacation & $0.2 \%$ & 218.22 \\
\hline
\end{tabular}

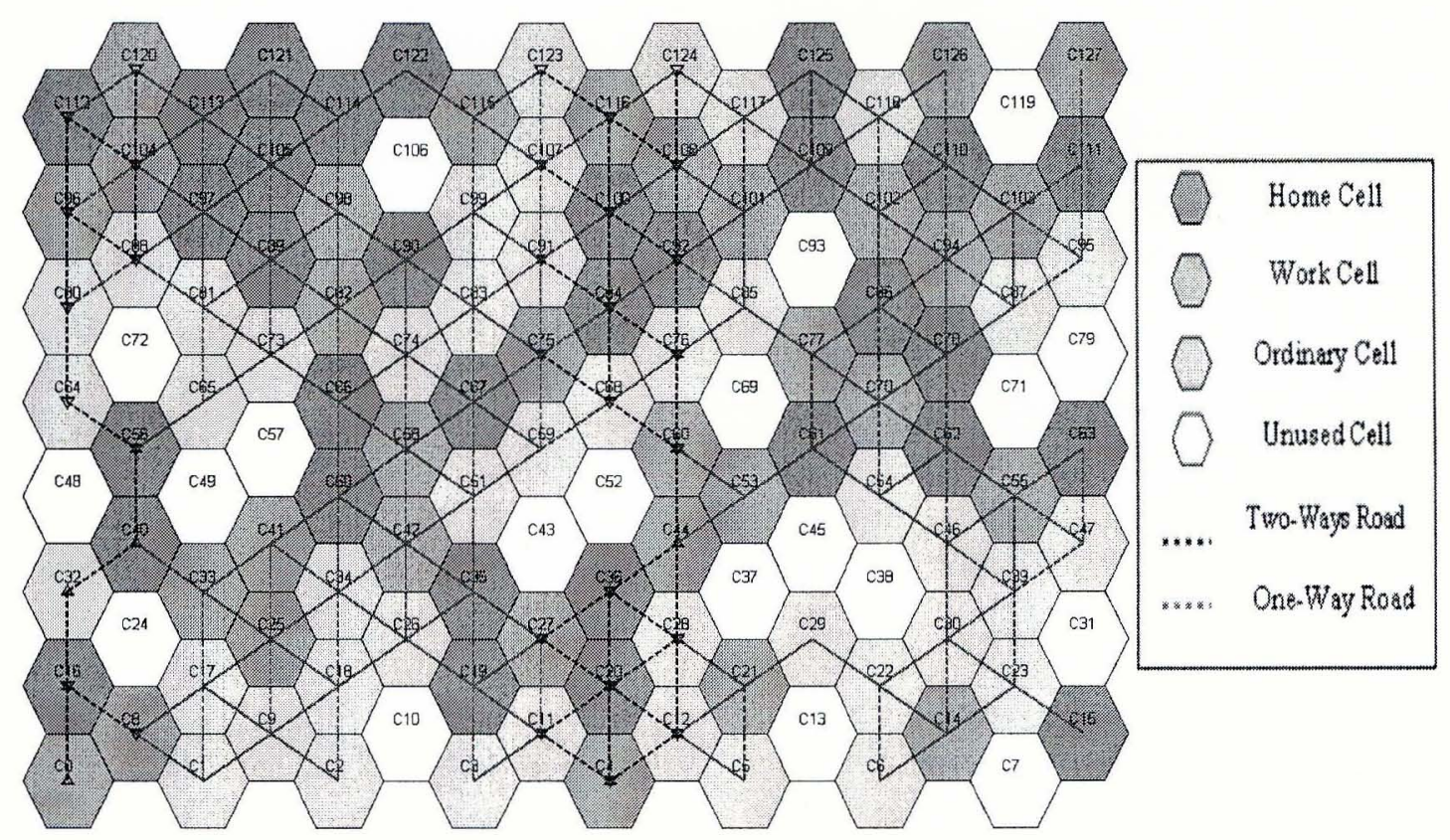

Fig. 7.1 Topology Model 


\subsection{Experimental Results for PCAC-RR}

The simulation was conducted to study the performance the proposed PCACRR scheme using different parameters that can affect the quality of the proposed technique. These parameters include the used mobility models (local, global, or combination), the number of time intervals used (ranging from one to six intervals) and the repeated sequences (with or without repeated sequences). This type of detailed analysis helped us to figure out what are the best parameters to be considered in the PCAC-RR technique. We conducted also the simulation to compare the performance of the proposed PCAC-RR (with the tuned parameters in the previous experiments) with other techniques. The details of all these experiments are explained here in details.

\subsubsection{Performance of PCAC-RR Using Different Types of Mobility Profiles}

The simulation was conducted to study the performance of the proposed PCAC-RR technique using different types of mobility profiles (models). We have three versions of the PCAC-RR technique according to the mobility model used [39]:

1. PCAC-RR(L): where the local mobility profiles are used alone to predict the next movements,

2. PCAC-RR(G): where the global mobility profiles are used alone to predict the next movements, and

3. PCAC-RR: where both local and global mobility profiles are used to predict the next movements. In this case, the global profile is used if the prediction given by the local profile has a low accuracy. 
The experiments have been performed for the three schemes using call arrival rate ranging from 0.01 up to 0.1 calls/second for each cell. The results of the CBP, CDP and BWU are shown in Figures 7.2, 7.3 and 7.4 respectively. As we can see from these figures, the combination (PCAC-RR) gives the best results by enhancing the accuracy of prediction and hence minimizing the dropping and the blocking probabilities. Also, this is reflected effectively on the bandwidth utilization results because the reservation of the bandwidth is done with higher accuracy. This of course reduces the waste in reserved bandwidth and allows more bandwidth for the new and additional handoff calls. From all of these results, we can conclude that the proposed PCAC-RR technique has best performance using the combination of the local and global mobility profiles, and it will be used in next phases of the performance study.

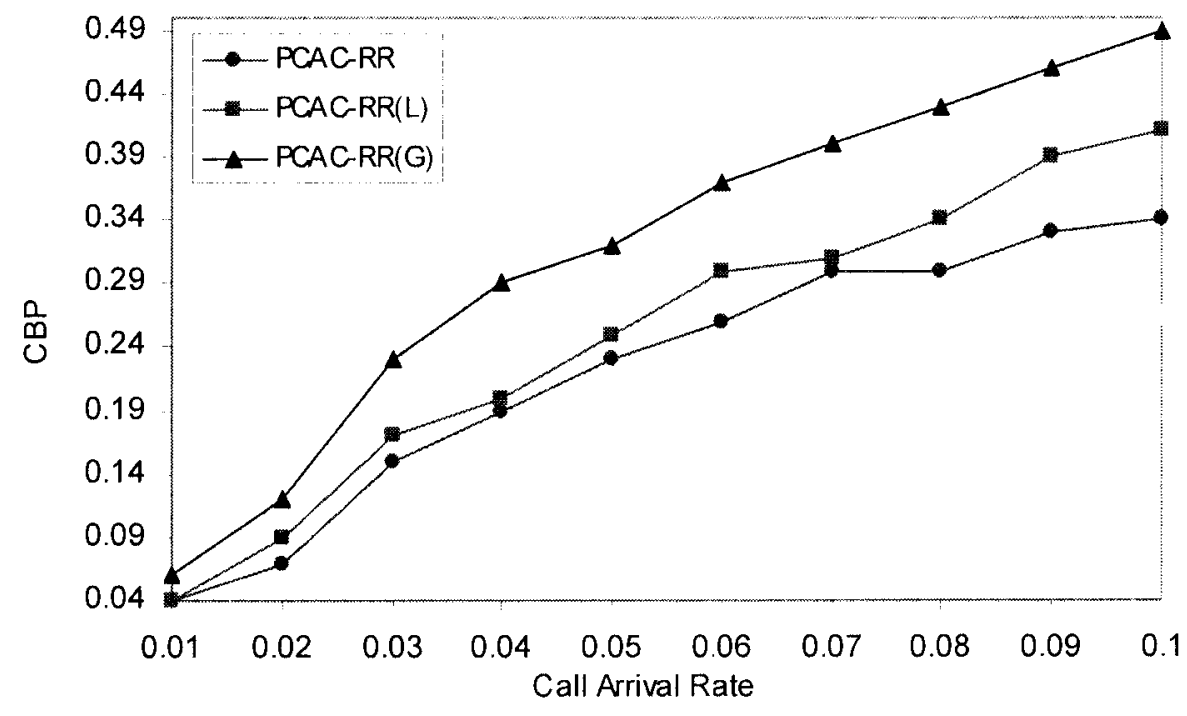

Fig. 7.2 Call blocking probability results comparing between the mobility models 


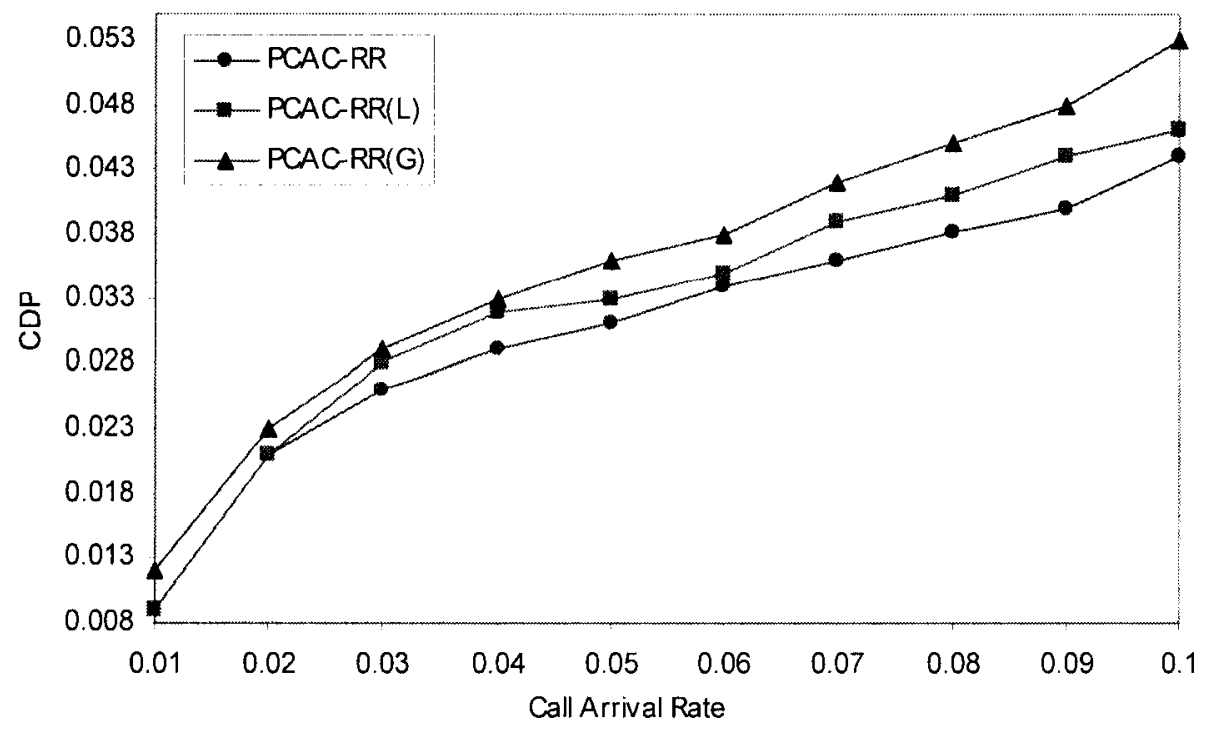

Fig. 7.3 Call dropping probability results comparing between the mobility models

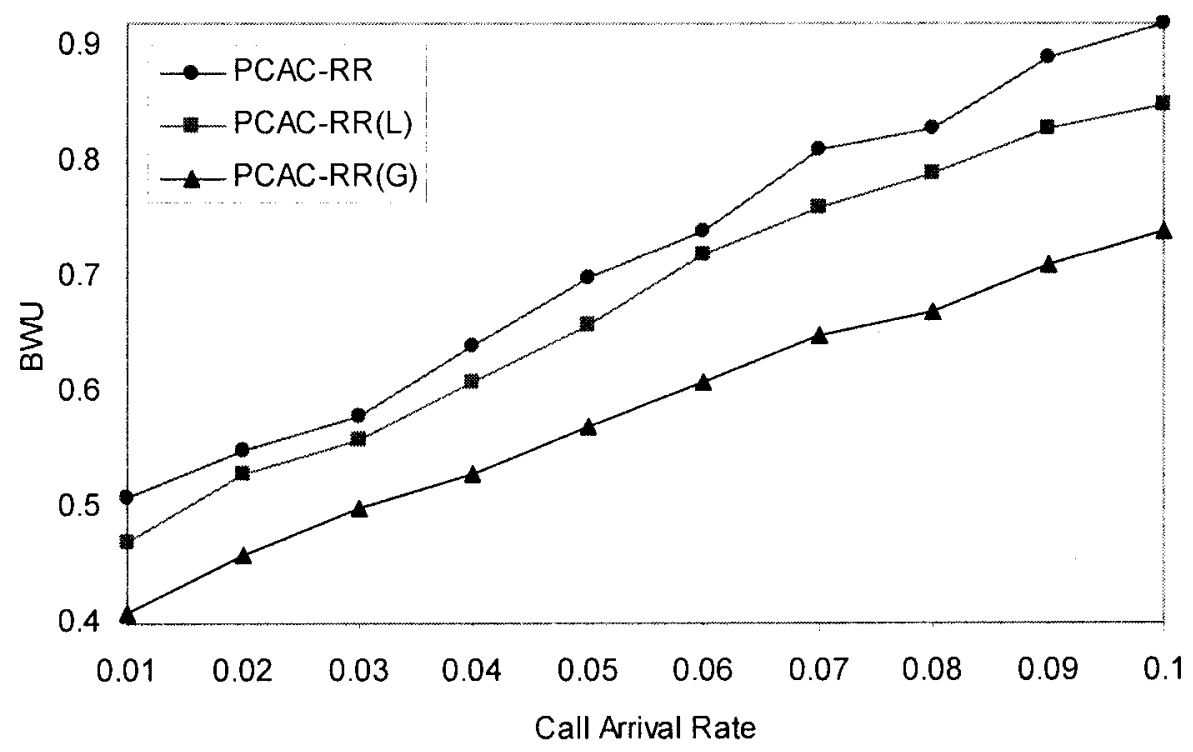

Fig. 7.4 Bandwidth utilization results comparing between the mobility models

\subsubsection{Performance of PCAC-RR Using Different Number of Day Time Intervals}

The simulation was also conducted to study the performance of the proposed

PCAC-RR technique using different number of day time intervals. The main idea is to 
see if there is a need to divide the day into intervals, and what will be the suitable number of intervals.

Reducing the number of time intervals will minimize the memory required for the profiles and it will reduce the time required to build and maintain the profiles. But at the same time, this may decrease the quality of prediction and increase the unnecessary bandwidth reservation. For example, if the number of intervals is minimized to be only one interval (this means the whole day without any division), then the frequent path in the morning will be also considered as a frequent path in the evening even if the mobile user never visited this path at evening. This will result in an unnecessary resource reservation and the overall performance will be affected. Reducing the number of time intervals may in some special cases enhance the prediction. For example, if the path was visited frequently at morning and at evening, then, making the whole day as one interval is better than dividing the day into two intervals (morning and evening) because using one interval will maximize probability of the frequent path which enhance the prediction.

Also, increasing the number of time intervals in some cases may cause some problems. For example, if we have the extreme with every hour of the day as one interval (then we have 24 time intervals!), this will affect tremendously the probability of the paths and we may not find frequent paths in each of these intervals.

Form this discussion, we can say that it is important to select the "suitable" number of time intervals according to the characteristic of the mobile environment. This means that the time intervals may be different form region to region, or form country to country according to their social and work culture. Also, it is not only the 
matter of how many time intervals exist, but it is also how they are distributed (from which time to which time). All of these issues should be taken into account into the design phase of the PCAC-RR technique.

To study the effect of the number of time intervals on the PCAC-RR, we conducted the simulation to perform six experiments using different number of time intervals $\left(\mathrm{N}_{\mathrm{T}}\right)$ in each of these experiments (from one time interval up to six time intervals). The used intervals are as shown in Table 7.3.

The experiments have been performed for call arrival rate ranging from 0.01 up to 0.1 calls/second for each cell. The results of the CBP, CDP and BWU are shown in Figures 7.5, 7.6 and 7.7 respectively. As we can see from these figures, the PCAC-RR performance is changing with number of periods. The best performance was obtained using four time intervals $\left(\mathrm{N}_{\mathrm{T}}=4\right)$. This means that dividing the day into four intervals (as shown in Table 6 at $\mathrm{N}_{\mathrm{T}}=4$ ) helped to find the frequent paths with the highest associated probabilities which improved the prediction process. This improvement helped to reserve the required amount of bandwidth according to the real need of the mobile user. That saves more bandwidth for other users, and gives better utilization of the limited resources. Therefore, in following experiments we use the PCAC-RR with $\mathrm{N}_{\mathrm{T}}=4$.

Table 7.3 The time intervals $\mathrm{T}_{\mathrm{i}}$ used in the experiments

\begin{tabular}{|c|c|c|c|c|c|c|}
\hline $\mathbf{N}_{\mathbf{T}}$ & $\mathbf{T}_{1}$ & $\mathbf{T}_{\mathbf{2}}$ & $\mathbf{T}_{\mathbf{3}}$ & $\mathbf{T}_{4}$ & $\mathbf{T}_{\mathbf{5}}$ & $\mathbf{T}_{\mathbf{6}}$ \\
\hline $\mathbf{6}$ & $6 \mathrm{am}-9 \mathrm{am}$ & $9 \mathrm{am}-12 \mathrm{pm}$ & $12 \mathrm{pm}-5 \mathrm{pm}$ & $5 \mathrm{pm}-6 \mathrm{pm}$ & $6 \mathrm{pm}-12 \mathrm{am}$ & $12 \mathrm{am}-6 \mathrm{am}$ \\
\hline $\mathbf{5}$ & $6 \mathrm{am}-10 \mathrm{am}$ & $10 \mathrm{am}-2 \mathrm{pm}$ & $2 \mathrm{pm}-6 \mathrm{pm}$ & $6 \mathrm{pm}-12 \mathrm{am}$ & $12 \mathrm{am}-6 \mathrm{am}$ & \\
\hline $\mathbf{4}$ & $6 \mathrm{am}-12 \mathrm{pm}$ & $12 \mathrm{pm}-6 \mathrm{pm}$ & $6 \mathrm{pm}-12 \mathrm{am}$ & $12 \mathrm{am}-6 \mathrm{am}$ & & \\
\hline $\mathbf{3}$ & $6 \mathrm{am}-2 \mathrm{pm}$ & $2 \mathrm{pm}-8 \mathrm{pm}$ & $8 \mathrm{pm}-6 \mathrm{am}$ & & & \\
\hline $\mathbf{2}$ & $6 \mathrm{am}-6 \mathrm{pm}$ & $6 \mathrm{pm}-6 \mathrm{am}$ & & & & \\
\hline
\end{tabular}




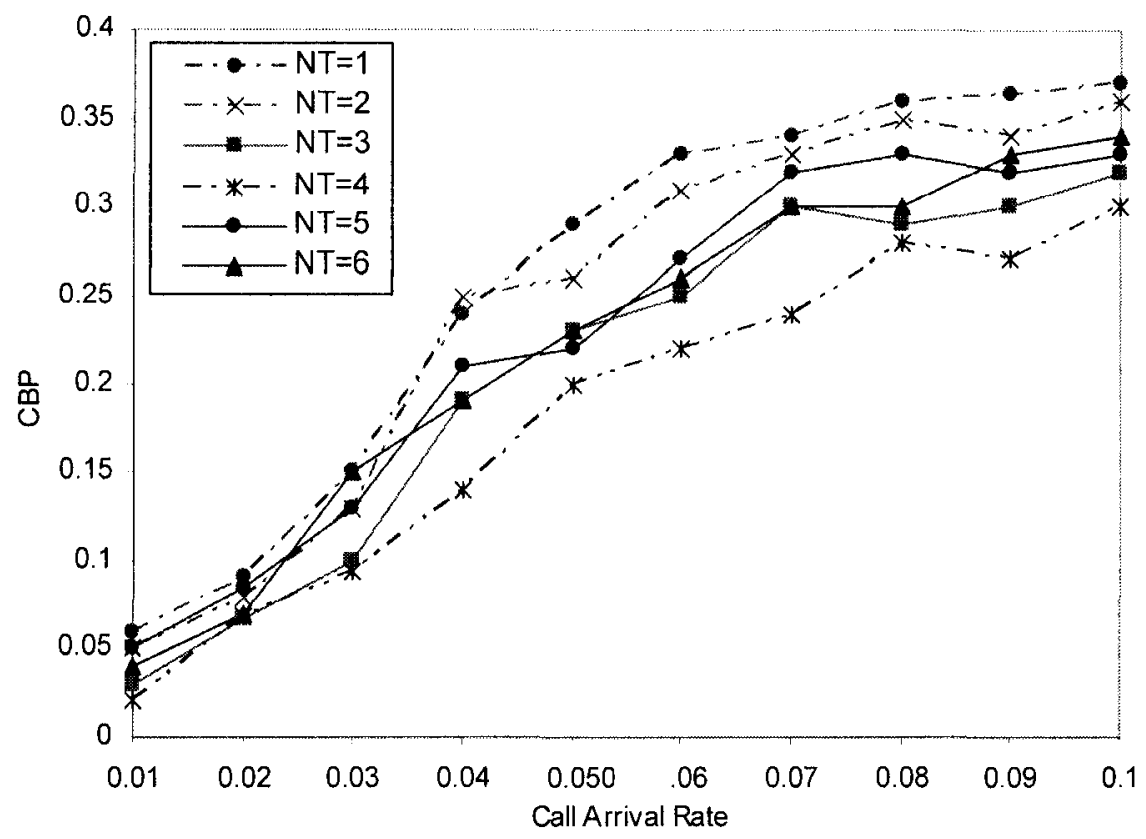

Fig. 7.5 Study the effect of the number of time intervals $\left(\mathrm{N}_{\mathrm{T}}\right)$ on the CDP for PCAC-RR technique

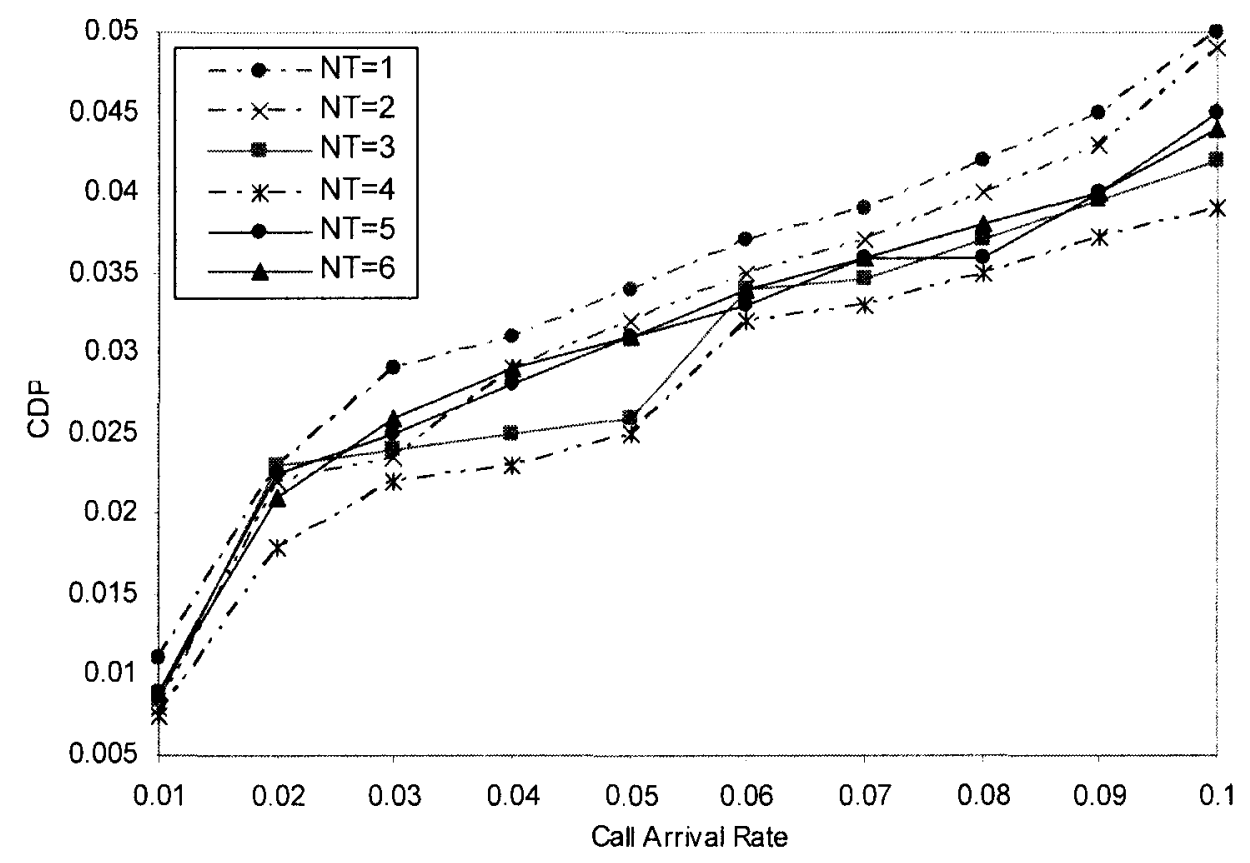

Fig. 7.6 Study the effect of the number of time intervals $\left(\mathrm{N}_{\mathrm{T}}\right)$ on the CDP for PCAC-RR technique 


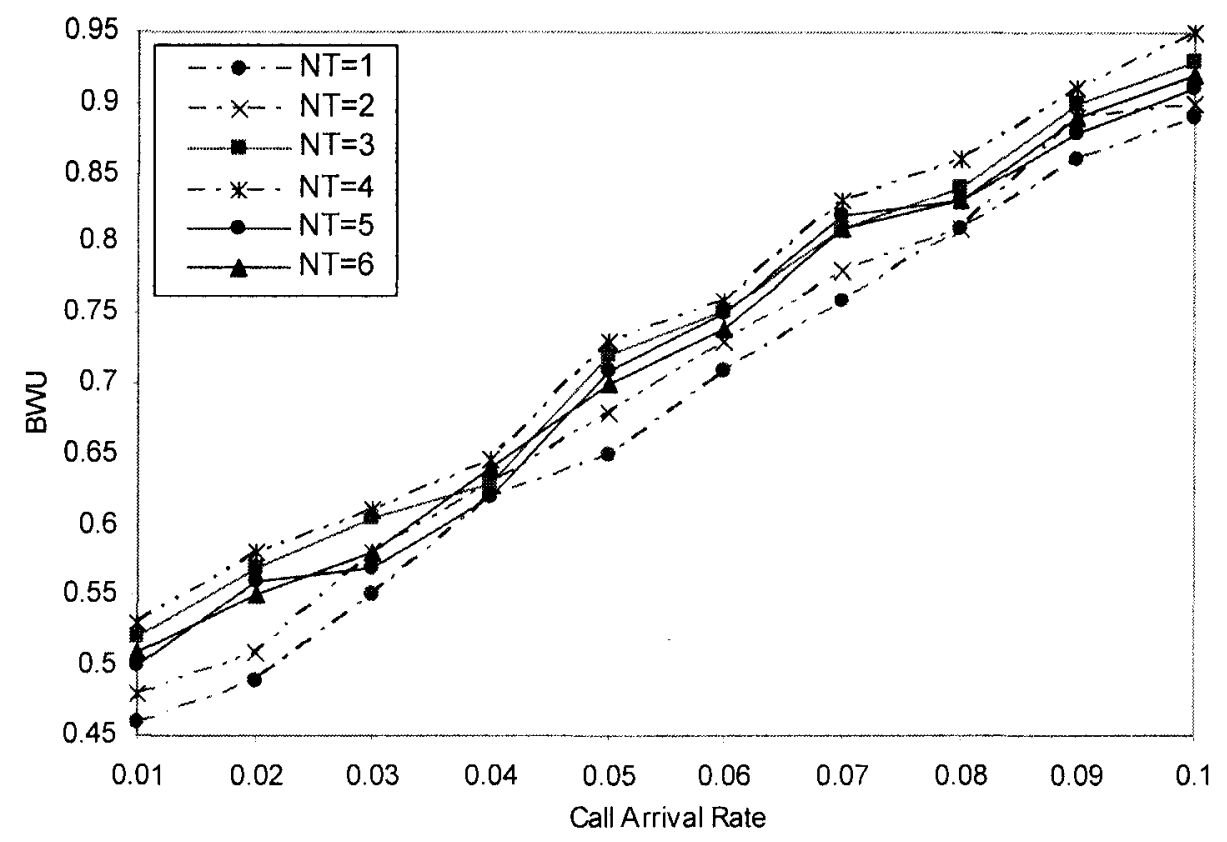

Fig. 7.7 Study the effect of the number of time intervals $\left(\mathrm{N}_{\mathrm{T}}\right)$ on the BWU for PCAC-RR technique

\subsubsection{Study of the Effect of Predicting the Handoff Time Using the Repeated Sequences}

The simulation was also conducted to study the effect of using the repeated sequences on the performance of the proposed PCAC-RR technique. As discussed in chapter 3, repeated sequences were generated by using the time slot $\Delta t$ to transform the base station into a sequence of base stations ID for each recorded visit. For example, if the $\mathrm{BS}_{1}$ was visited from $5: 30$ to $5: 33$ then using $\Delta t=l$ minute $\mathrm{BS}_{1}$ is converted to the sequence $\left\{\mathrm{BS}_{1}, \mathrm{BS}_{1}, \mathrm{BS}_{1}\right)$. We want in this section to answer the following question: "is it really necessary to use the repeated sequences proposed in PCAC-RR technique?". To answer this question, we conducted the simulation to perform four experiments on the PCAC-RR technique. In the first three experiments, we use the PCAC-RR with different values of $\Delta t$ (we used $\Delta t=1$ minute, $\Delta t=5$ 
minutes, and $\Delta t=10$ minutes). In the fourth experiment, we did not use the time slot (we called this approach PCAC-RR without repeated sequences).

The results are shown in Fig. 7.8, 7.9, and 7.10. The diagrams show that using $\Delta t=1$ minute gives the best results. Using $\Delta t=10$ minutes gives the worst results (even compared to the results of the PCAC-RR without repeated sequences). This gives us an indication that using the suitable value of $\Delta t$ is very critical for the PCAC-RR technique because this allows predicting correctly the handoff time. Using large value of $\Delta t$ will not help in predicting correctly when the user leaves the current base station in a short time compared to $\Delta t$. In such cases large value of $\Delta t$ will force the PCACRR technique to produce wrong information about the handoff time, which affects tremendously the overall performance of the PCAC-RR technique. On the other hand, using smaller value of $\Delta t$, will improve the prediction of the handoff time. This can be seen when we use $\Delta t=1$ minute and $\Delta t=5$ minutes. In these cases, the cells that have been visited for long time will be reflected in the frequent paths correctly and we will be able to make the resource reservation at the correct time.

In addition, the results indicate that without implicit time in these sequences (where we use PCAC-RR without repeated sequences), we were not able to predict when the user will leave the current base station, and this resulted in extra overhead on the limited bandwidth. In this case we may reserve the bandwidth very early resulting in more dropped and blocked calls. In addition BWU is also low for this case due to wasted $\mathrm{BW}$ reservations. From Fig. 7.8, 7.9, and 7.10, we can say that using repeated sequences is important to predict the handoff time which enhance the overall performance of PCAC-RR technique (especially with high call arrival rates). 
Therefore, in subsequent experiments, we continue to use the repeated sequences with PCAC-RR technique.

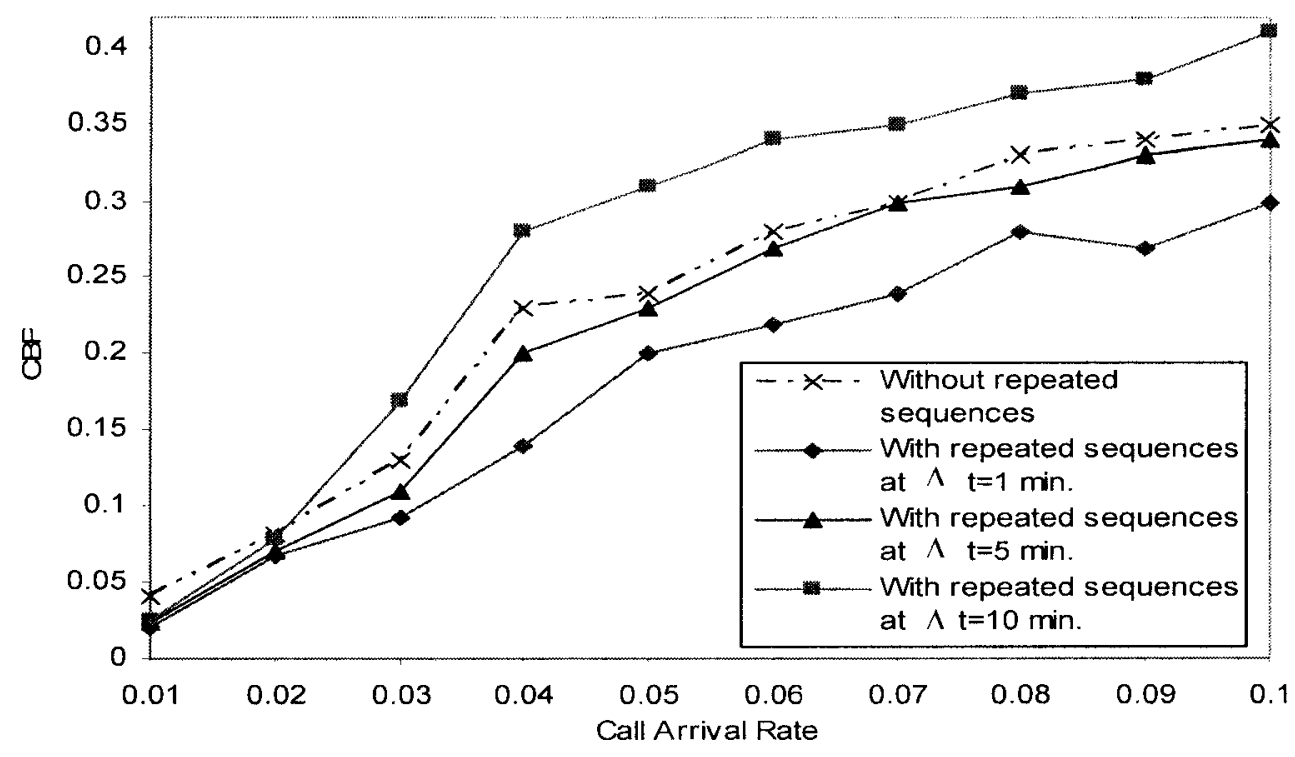

Fig. 7.8 Study of the effect of using the repeated sequences on the CBP for PCACRR technique

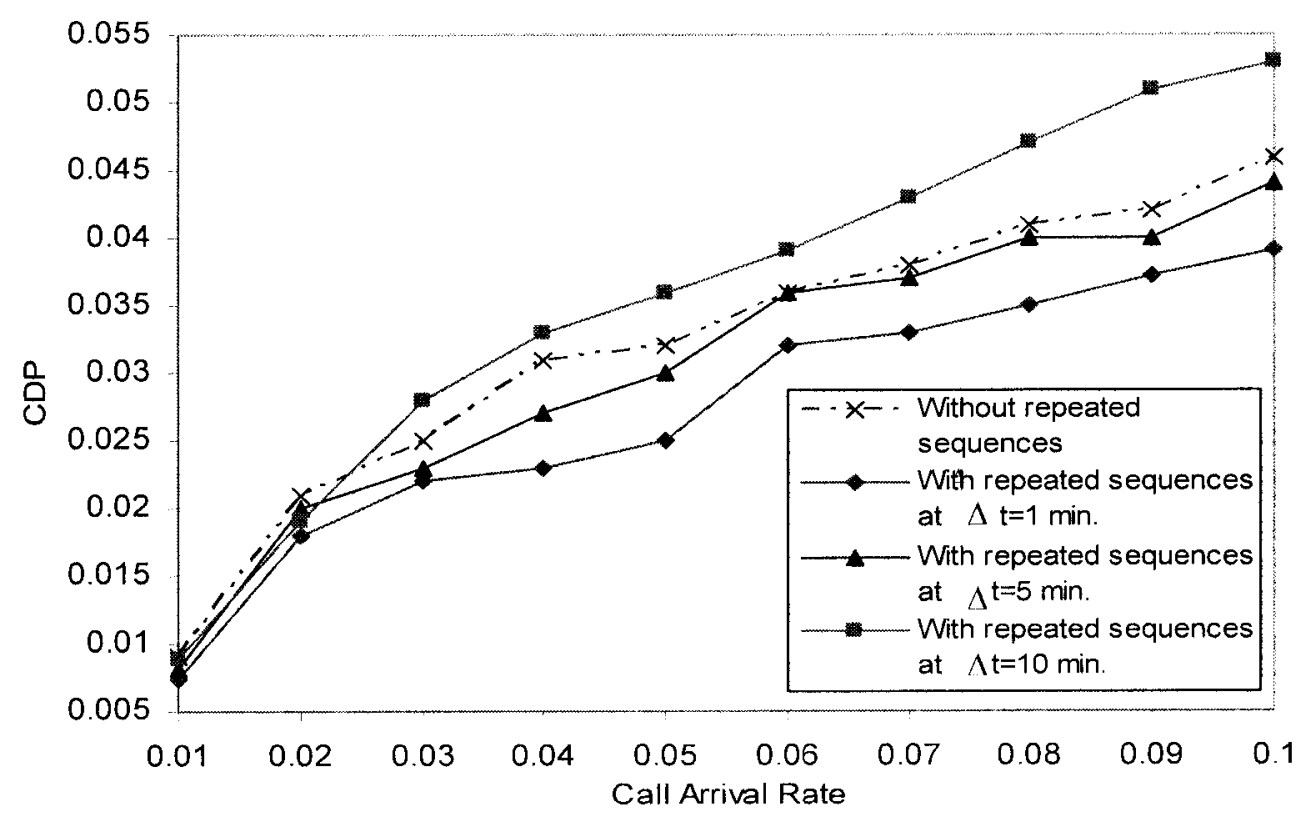

Fig. 7.9 Study of the effect of using the repeated sequences on the CDP for PCACRR technique. 


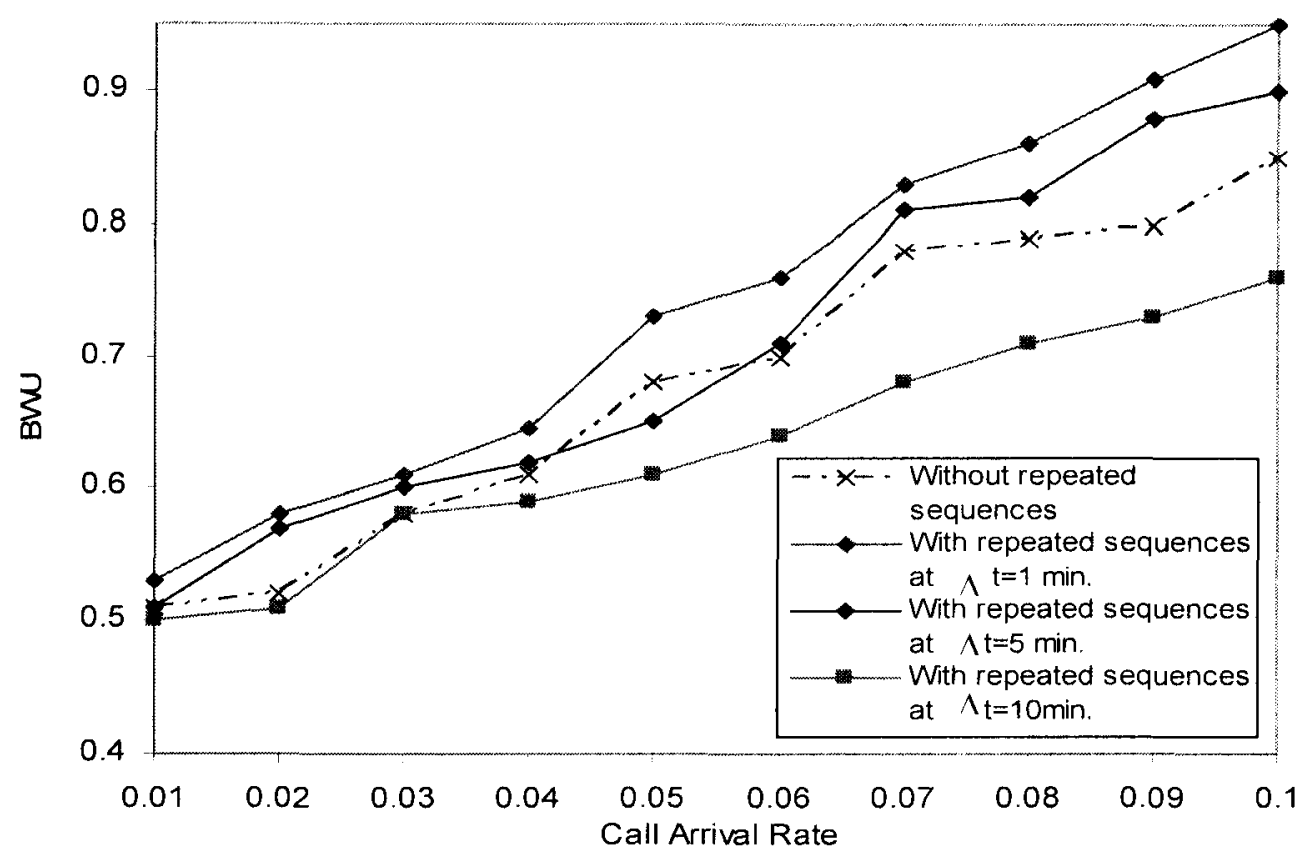

Fig. 7.10 Study of the effect of using the repeated sequences on the BWU for PCAC-RR technique

\subsubsection{Comparison between the PCAC-RR Technique and Other Techniques}

The simulation was also conducted to compare performance of the proposed PCAC-RR scheme with PR-CAT4 and FR-CAT2 schemes that are described in [27, 68]. PR-CAT4 is a predictive scheme and it takes into account the movement to the next cell from the previous cell through the current cell. In FR-CAT2 scheme there is no prediction and a fixed portion of the total bandwidth is reserved for the handoff calls.

The experiments have been performed for the three techniques using call arrival rate ranging from 0.01 up to 0.1 calls/second for each cell. The combined mobility model of the PCAC-RR technique is used with four time intervals $\left(\mathrm{N}_{\mathrm{T}}=4\right)$, 
and using the repeated sequences ( $\Delta t=1$ minutes). The results are shown in the figures from Fig. 7.11 up to Fig. 7.16.

Figure 7.11 and 7.12 show the resultant CBP for each technique and their comparison. The PCAC-RR algorithm has the lowest CBP for each call arrival rate. Figure 7.12 shows the percentage of improvement in the CBP for the PCAC-RR technique compared to the PR-CAT4 technique. We can see that there a noticeable enhancement in the performance of the PCAC-RR. This result is due to the fact that the improved prediction of the future movement(s) allows us to free more $\mathrm{BW}$ on the cells that are expected to be visited with low probabilities, and this BW is available for the new calls. Also, we can see that the proposed PCAC-RR technique has a better performance than the PR-CAT4 technique because the prediction is enhanced by using local and global profiles, and by using the time of request as an important factor [37].

Figures $7.13,7.14,7.15$ and 7.16 show that the proposed PCAC-RR technique has the lowest CDP and the highest bandwidth utilization compared to the other techniques. This is because in the proposed algorithm, we predict a longer path (not only the next step as in PR-CAT4). This allows us to give advance notice of reservations for suitable amount of BW for the handoff calls in expected cells. We can see form Figures 7.12, 7.14 and 7.16 that we have a significant improvement on the $\mathrm{CDP}$ and the BWU (around 27\%). 


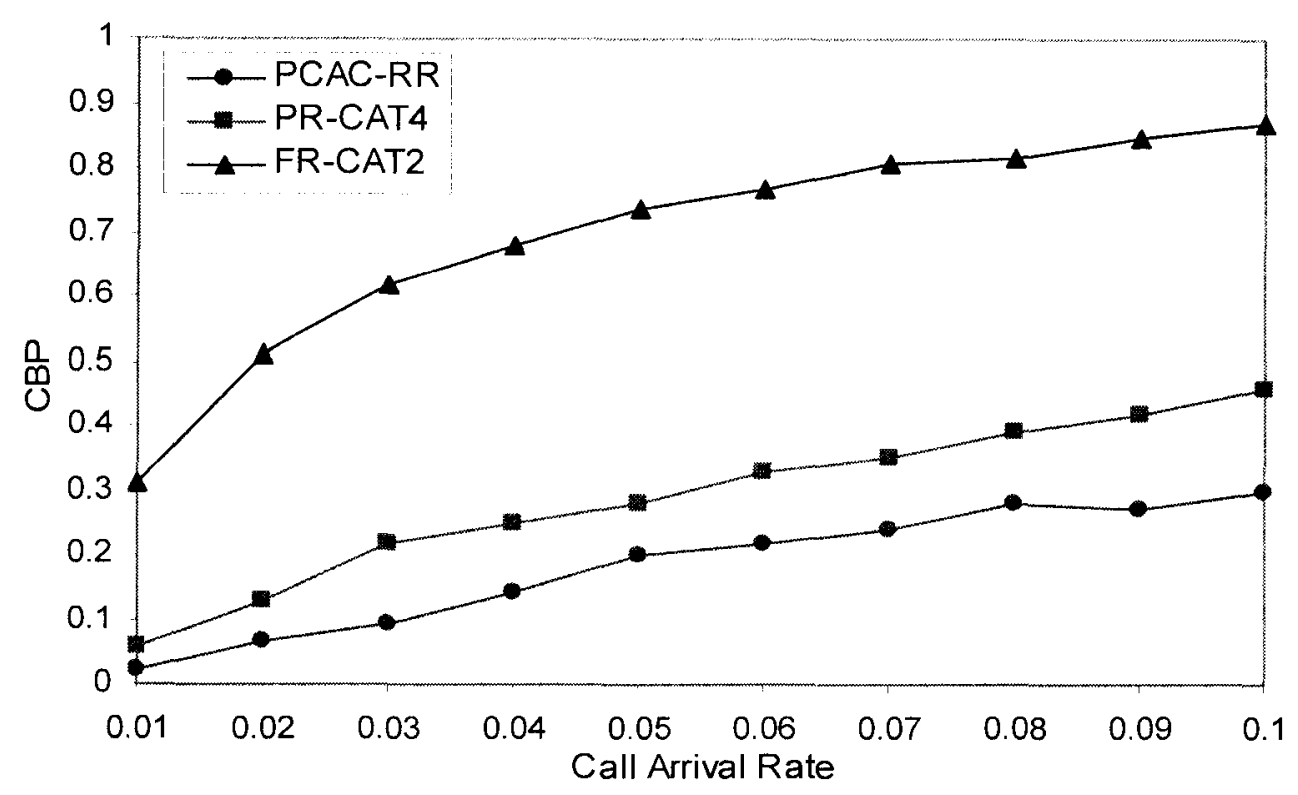

Fig. 7.11 Call blocking probability results comparing between different schemes

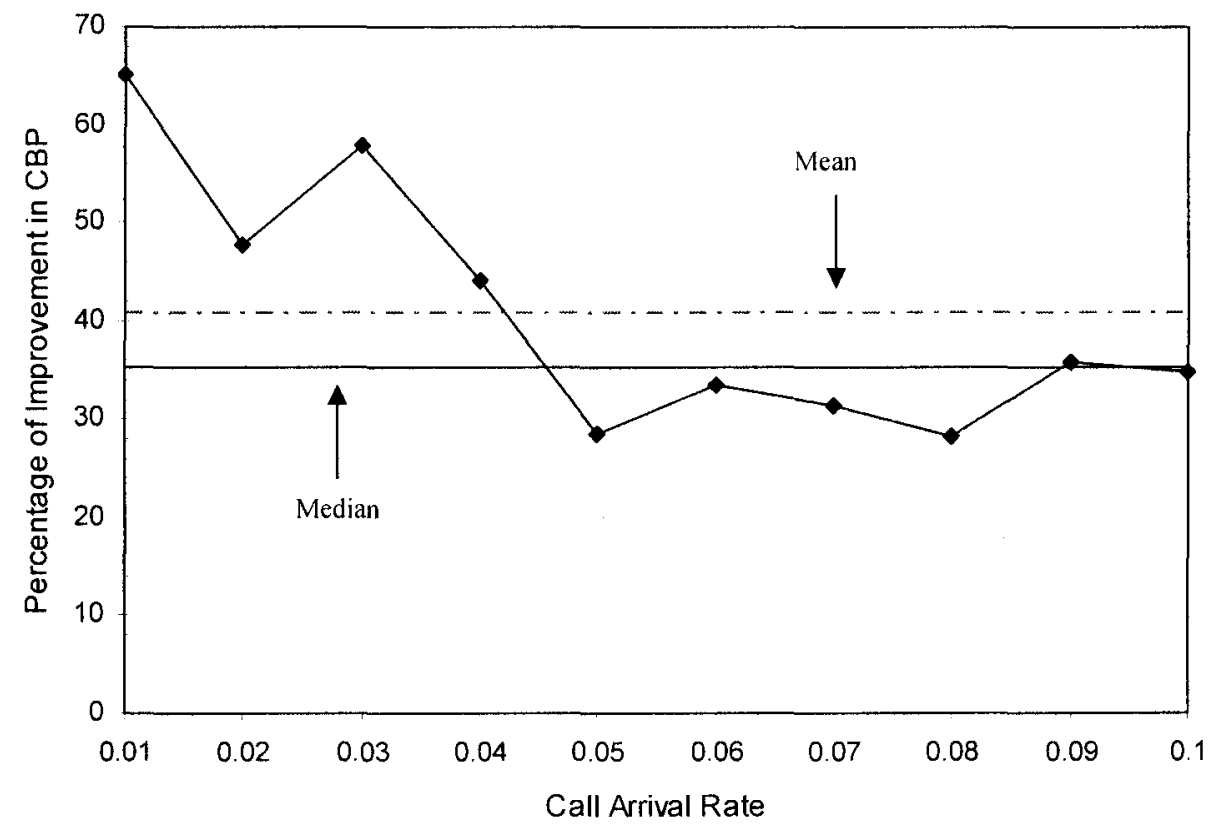

Fig. 7.12 Percentage of improvement in the CBP for PCAC-RR comparing to PRCAT4 


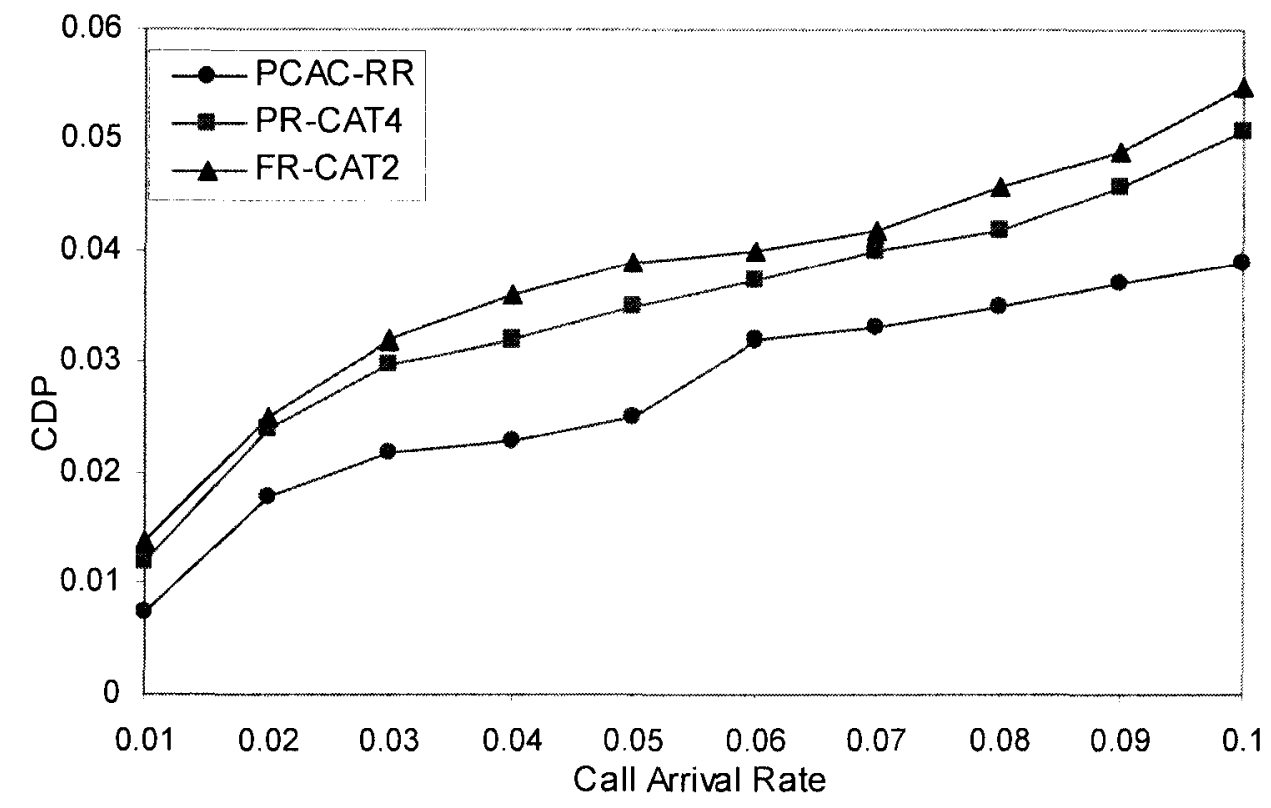

Fig. 7.13 Call dropping probability results comparing between different schemes

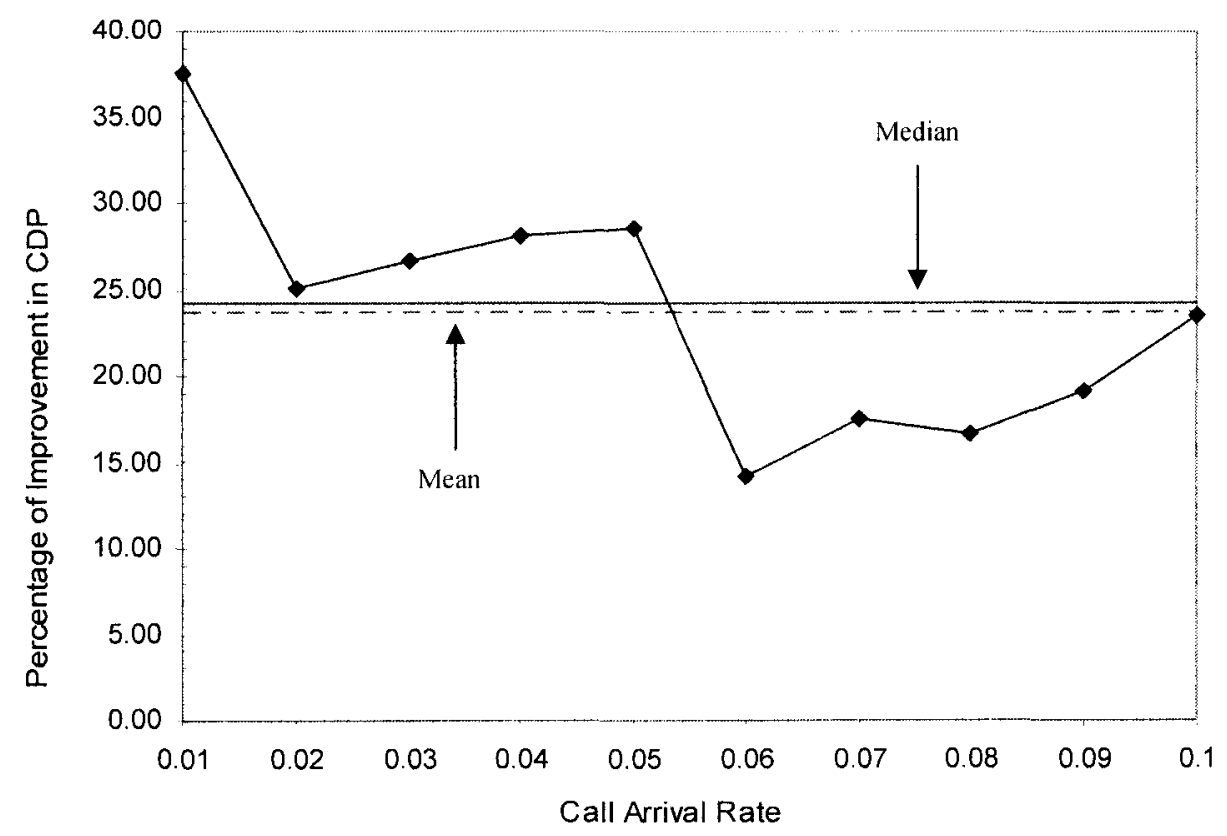

Fig. 7.14 Percentage of improvement in the CDP for PCAC-RR comparing to PRCAT4 


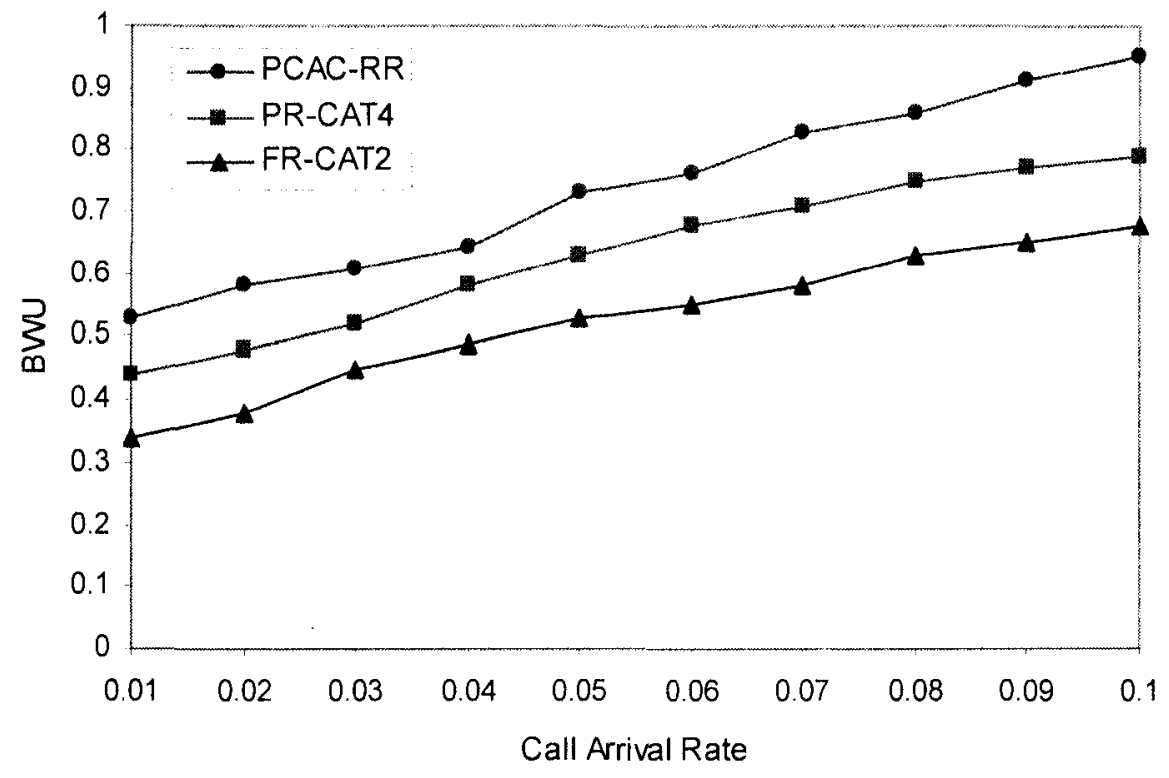

Fig. 7.15 Bandwidth utilization results comparing between different schemes

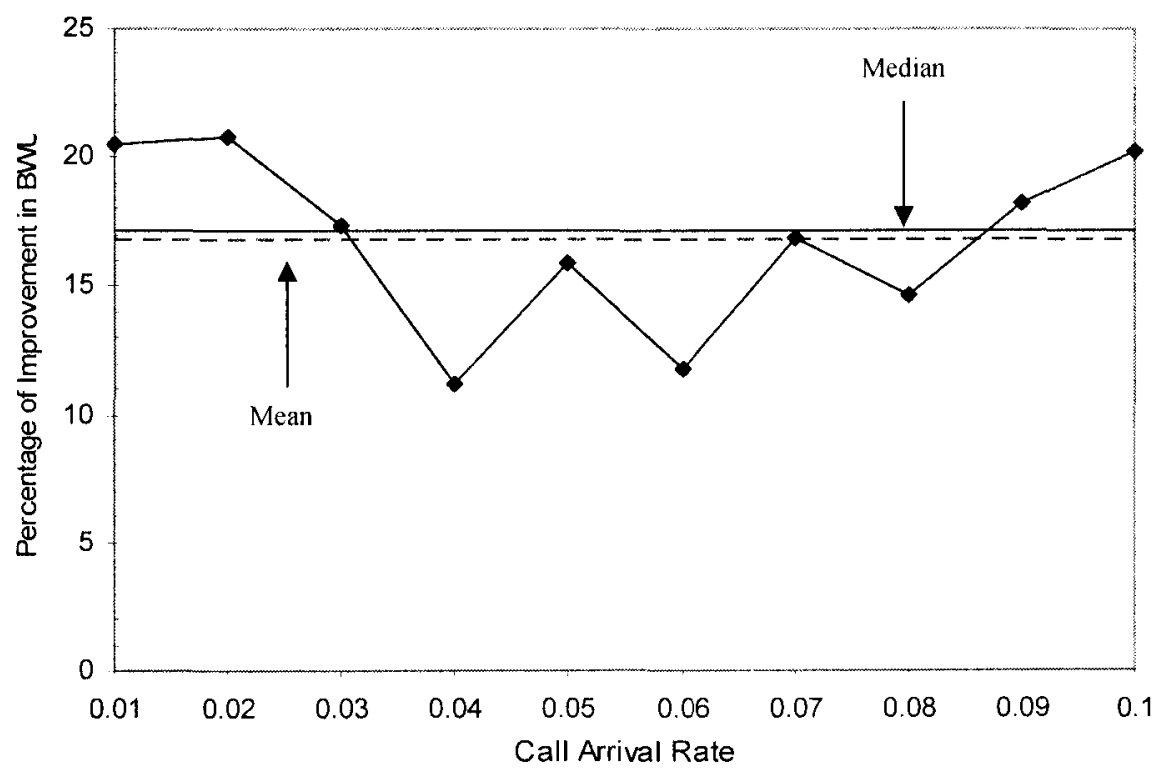

Fig. 7.16 Percentage of improvement in the CDP for PCAC-RR comparing to PRCAT4 


\subsection{Experimental Results for MSP-CACRR}

The simulation was conducted to evaluate the performance of the proposed MSP-CACRR technique and compare its performance with other predictive techniques. We compare the performance of the MSP-CACRR with three other schemes: PCAC-RR scheme, PR-CAT4 scheme (described in [28]) and the FR-CAT2 scheme (described in [28] and [69]).

It has been demonstrated that the best number of time intervals that can be used for the users of the simulated mobile networks is four time periods [70, 71]. Therefore, in all of the following experiments, we will use these four time intervals of the day: T1: $[6: 00 \mathrm{am}-12: 00 \mathrm{pm}], \mathrm{T} 2:[12: 00 \mathrm{pm}-6: 00 \mathrm{pm}], \mathrm{T} 3:[6: 00 \mathrm{pm}-12: 00$ $\mathrm{am}]$, and T4: [12:00 pm - 6:00 am]. The updated simulation parameters are as shown in Table 7.4.

In the first set of experiments, we study the effect of different factors on the MSP-CACRR technique. In the next set of experiments, we compare its performance with the other techniques.

\subsubsection{Study of the Effect of Using MobilePrefixSpan for Mining the Sequential Patterns}

We conducted the simulation to compare between the performance of the PCAC-RR technique and the performance of the MSP-CACRR $\left(\mathrm{d}_{1}\right)$ technique when we use only one dimension which is the is the time intervals of the day and ignore the second two dimensions. Using only the number of time interval will enable us to study the effect of using the MobilePrefixSpan as a sequence mining technique. This is because in the PCAC-RR technique we also used only the time intervals of the day. 
Table 7.4 Updated Simulation Parameters

\begin{tabular}{|l|l|}
\hline \multicolumn{1}{|c|}{ Parameter } & \multicolumn{1}{|c|}{ Value } \\
\hline Number of cells & 128 cells \\
\hline Type of cells & Hexagonal cells \\
\hline $\begin{array}{l}\text { Diameter of } \\
\text { each cell }\end{array}$ & $1 \mathrm{mi}$ \\
\hline NT & $\begin{array}{l}4 \text { intervals: T1: [6:00 am }-12: 00 \mathrm{pm}], \text { T2: }[12: 00 \mathrm{pm}-6: 00 \mathrm{pm}] \\
\text { T3: }[6: 00 \mathrm{pm}-12: 00 \mathrm{am}], \text { and T4: [12:00 pm - } 6: 00 \mathrm{am}] .\end{array}$ \\
\hline$\Delta \mathrm{t}$ & 1 minute \\
\hline min_support & 200 \\
\hline Call arrival rate & $\begin{array}{l}\text { Poisson distribution with mean } \lambda \text { (varies from } 0.01 \text { to } 0.1 \\
\text { calls/second) }\end{array}$ \\
\hline $\begin{array}{l}\text { Call duration } \\
\text { time }\end{array}$ & Exponentially distribution with mean $\mu=180$ seconds \\
\hline $\begin{array}{l}\text { Maximum } \\
\text { Speed }\end{array}$ & 60 mi/h \\
\hline $\begin{array}{l}\text { Channel } \\
\text { capacity of each } \\
\text { cell }\end{array}$ & 40 bandwidth units (BU) \\
\hline $\begin{array}{l}\text { BW required by } \\
\text { a voice call }\end{array}$ & 1 BU \\
\hline $\begin{array}{l}\text { BW required by } \\
\text { a text call }\end{array}$ & 2 Bus \\
\hline $\begin{array}{l}\text { BW required by } \\
\text { a audio call }\end{array}$ & 5 Bus \\
\hline $\begin{array}{l}\text { BW required by } \\
\text { a video call }\end{array}$ & 10 Bus \\
\hline
\end{tabular}

The simulation results for the three performance measures (CBP, CDP, and BWU) are shown in Fig. 7.17, 7.18, and 7.19. We can see from these results that using the MobilePrefixSpan technique helped to enhance the overall performance especially the CDP measure as shown in Fig. 3. The main reason for this enhancement is that using MobilePrefixSpan with the MSP-CACRR $\left(\mathrm{d}_{1}\right)$ technique enables us to discover new subsequences that could not be discovered using the PCAC-RR [70, 71]. For example, if the minimum support is equal to 2 and each of

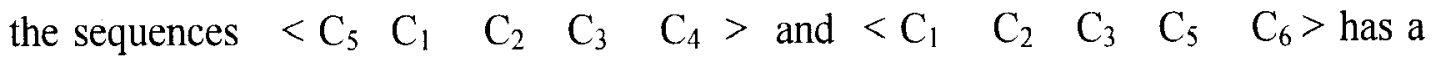


frequency of 1 . This means that both of them are not frequent. In the mobile data mining technique used in PCAC-RR, we were unable to discover any other subsequence from these two sequences. But when we use MobilePrefixSpan in MSP$\operatorname{CACRR}\left(\mathrm{d}_{1}\right)$ technique, we discover that there is an important subsequence $<\mathrm{C}_{1} \quad \mathrm{C}_{2}$ $\mathrm{C}_{3}>$ which is repeated in both sequences and it will be a frequent sequence (has at least support equal to 2). This means that in the PCAC-RR, we can miss some important patterns of the user behavior and in this case the perdition will not be done correctly while in MSP-CAC-RR $\left(\mathrm{d}_{1}\right)$ we were able to find these missing patterns using MobilePrefixSpan. This has a significant influence on the handoff calls where we can accurately predict the frequent sequences and make the necessary BW reservations to complete the calls without interruptions.

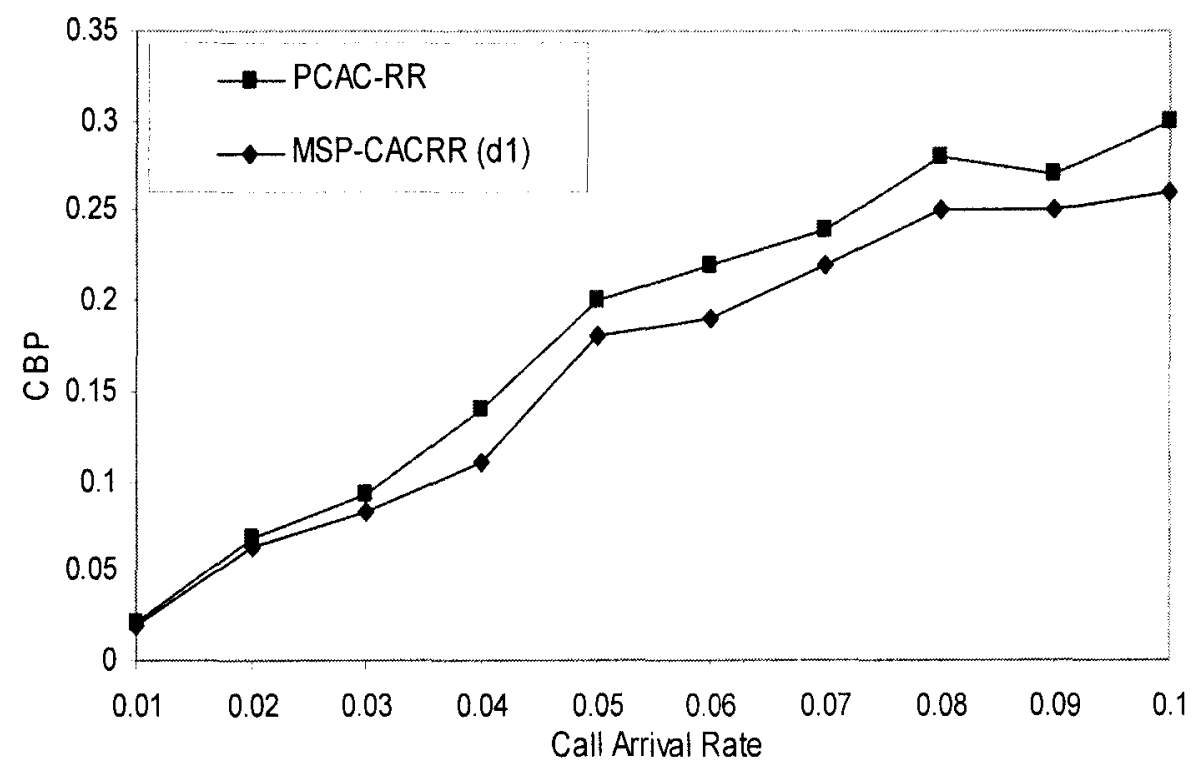

Fig. 7.17 Call blocking probability results comparing between MSP-CACRR $\left(\mathrm{d}_{\mathrm{l}}\right)$ and PCACRR 


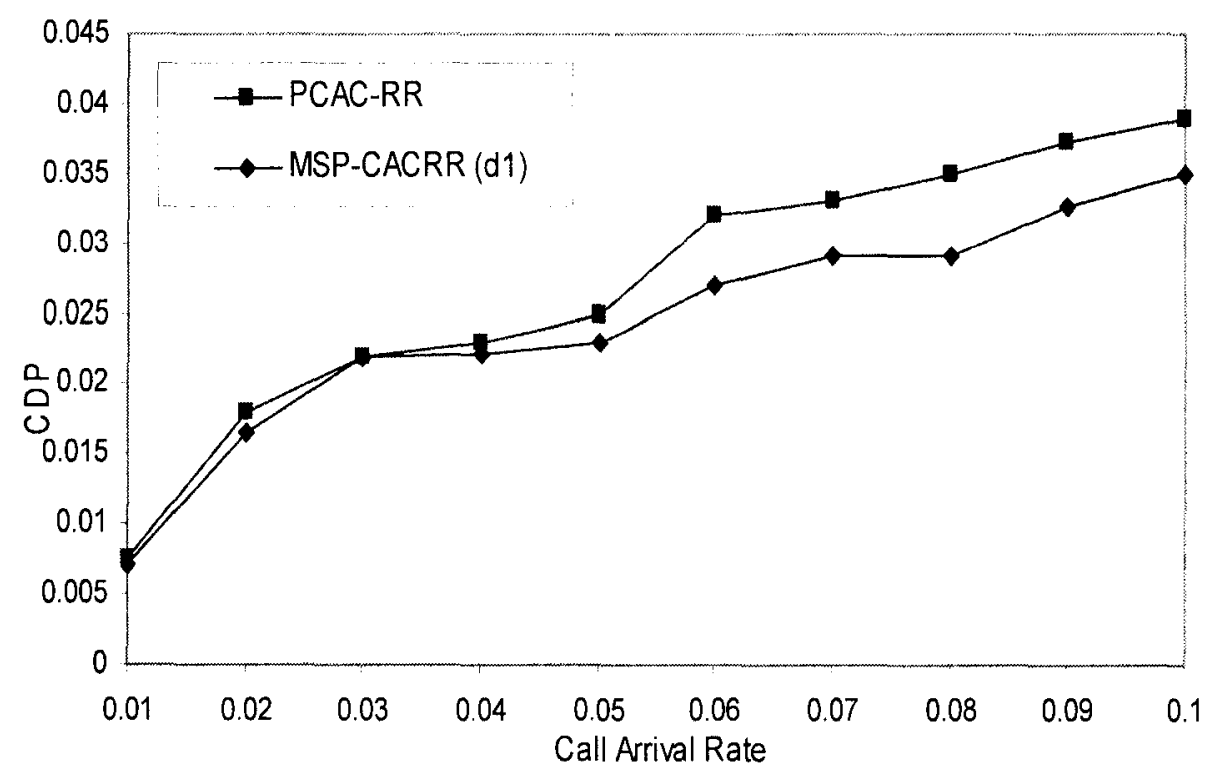

Fig. 7.18 Call dropping probability results comparing between MSP$\operatorname{CACRR}\left(\mathrm{d}_{1}\right)$ and PCACRR

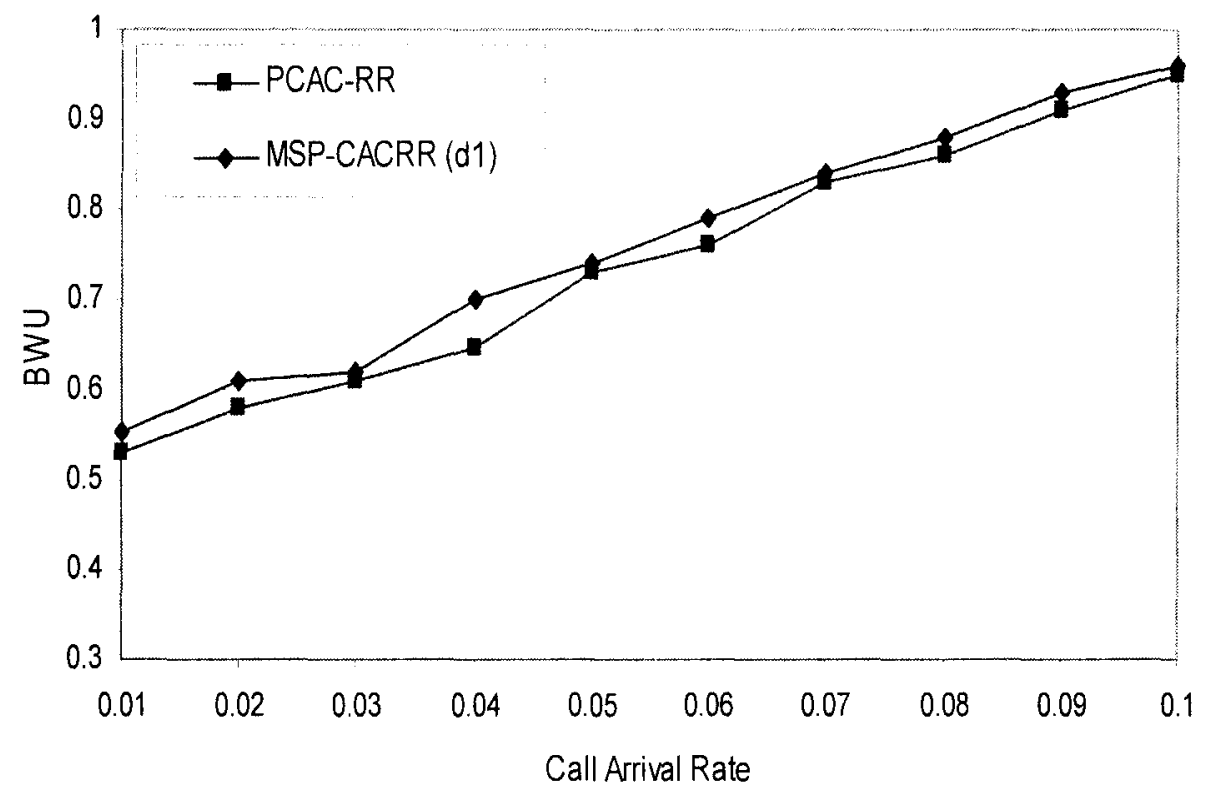

Fig. 7.19 Bandwidth utilization results comparing between MSP-CACRR $\left(d_{1}\right)$ and PCACRR 


\subsubsection{Study the Effect of Using the Type of Service as One of the Dimensions}

The simulation was also conducted to study the effect of using the type of service in the MSP-CACRR technique. In this case, we do not only use information about the time interval of the day (which has been proven to have better performance from the previous experiment), but we also use information about the type of the services as explained in chapter 5. We refer to the used technique as MSP$\operatorname{CACRR}\left(d_{1}, d_{3}\right)$ where we add the dimension $d_{3}$ which refers to the type of used services. As we can se from the results sown in Fig. 7.20, 7.21, and 7.22. We can see that using the type service as an additional dimension will not enhance the performance, but it will make a clear negative effect on the performance as shown from the results. This can be explained by understanding the type of new information we can obtain from recording the type of the services and its relation to the user's transition from one cell to another. Recording the type of the service in this case, will not useful (but it may be misleading) since the transition form one cell to another will not be affected by type of the services. This is because all of the cells in the network can provide the all of the services from Class-I and Class-II. This means that the mobile user may use the same sequence of cells with both classes of the services. In other words, we may not be able to detect the correct frequent sequences (with the correct support) if we use the service type to differentiate between the sequences. We can here highlight the fact that the type of the service may be useful in other cases, where the transition from one cell to another (or from one connection point another connection point in the network) is affected by the type of the service. This is not the situation in our case where all of the cells can be used for all type of services. Therefore we will not consider the type of the service as an additional dimension. 


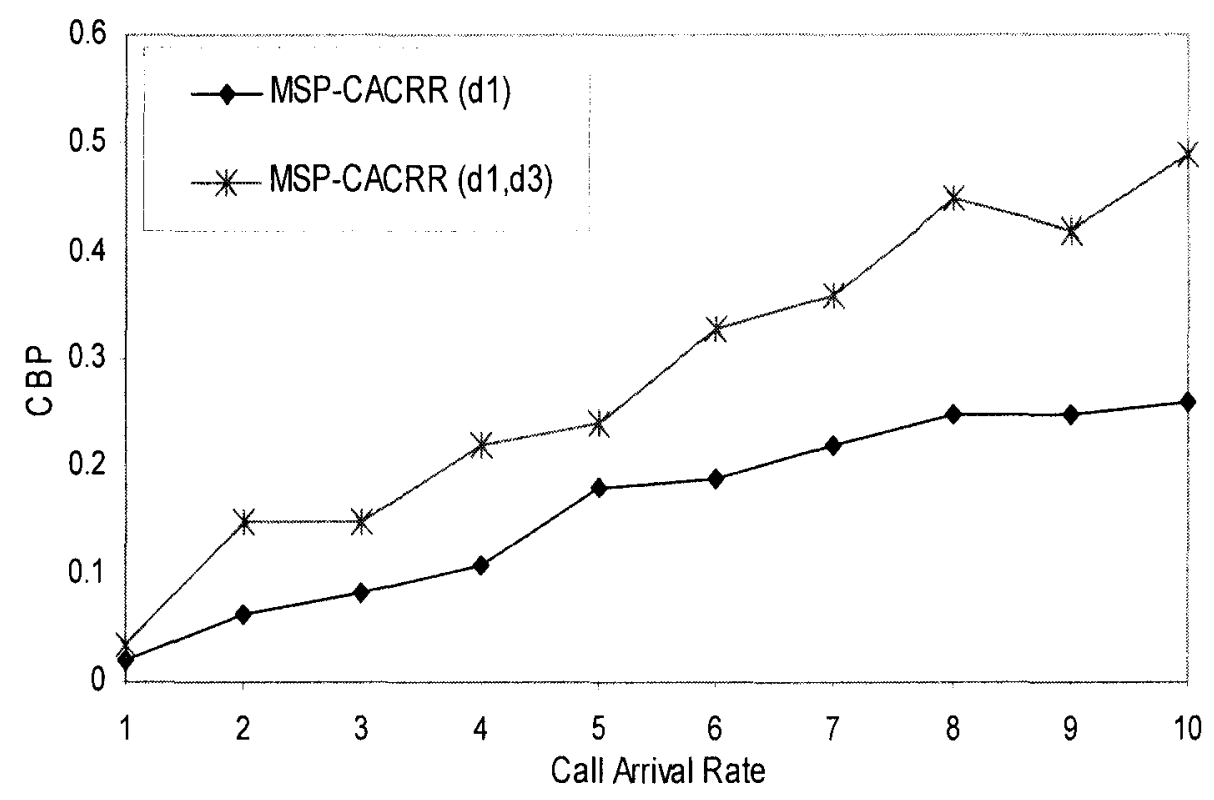

Fig. 7.20 Call blocking probability results comparing between MSP-CACRR $\left(\mathrm{d}_{1}\right)$ and MSP-CACRR $\left(\mathrm{d}_{1}, \mathrm{~d}_{3}\right)$

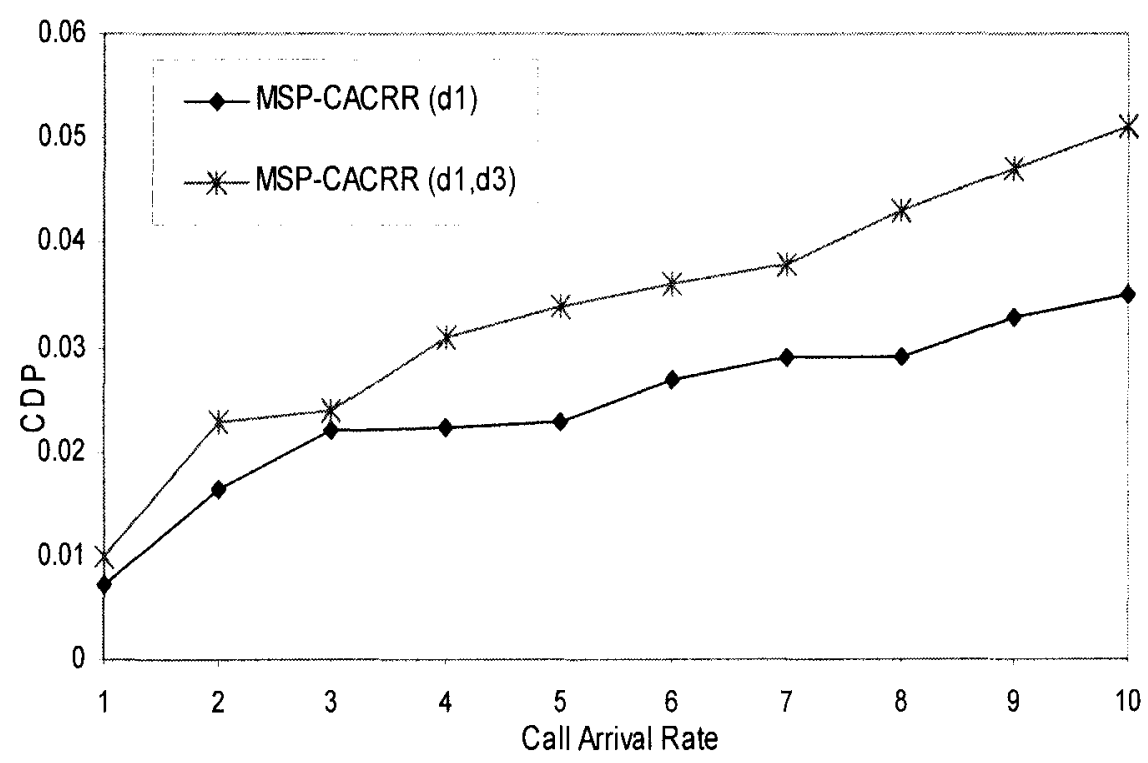

Fig. 7.21 Call dropping probability results comparing between MSP$\operatorname{CACRR}\left(\mathrm{d}_{1}\right)$ and MSP-CACRR $\left(\mathrm{d}_{1}, \mathrm{~d}_{3}\right)$ 


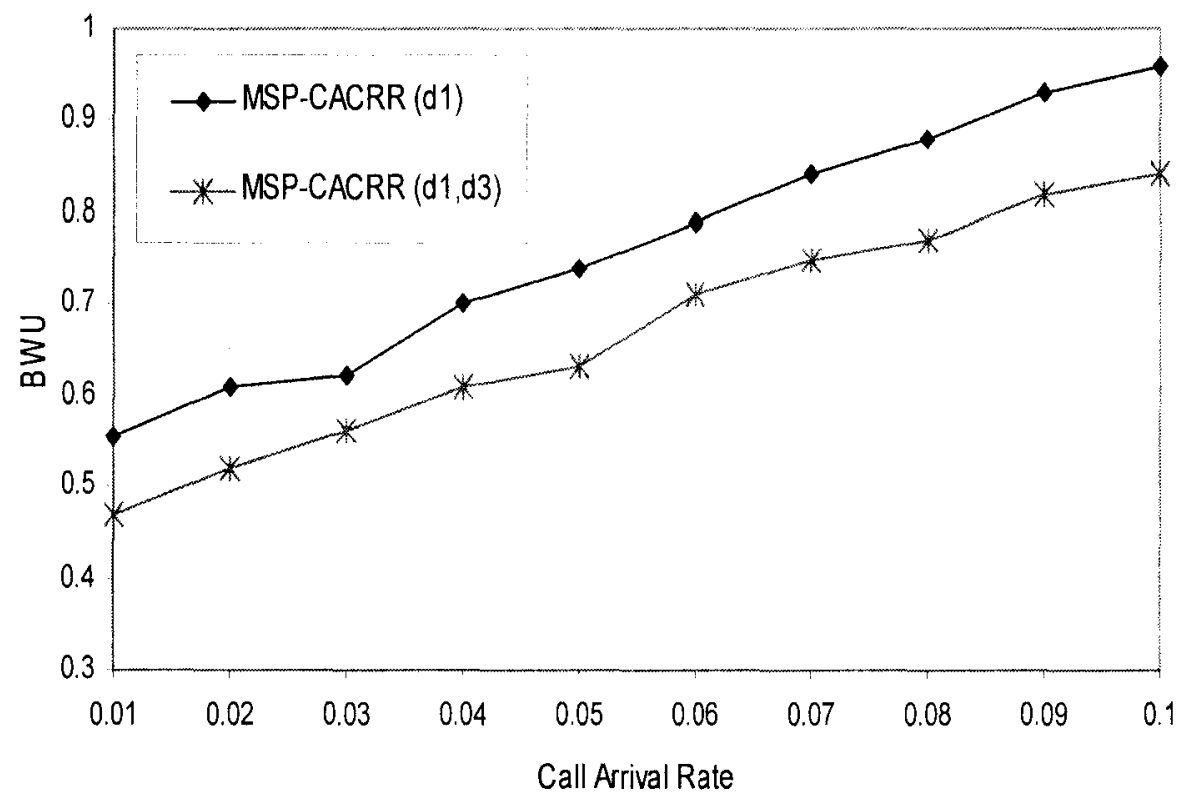

Fig. 7.22 Bandwidth utilization results comparing between MSP-CACRR $\left(\mathrm{d}_{1}\right)$ and MSP-CACRR $\left(\mathrm{d}_{1}, \mathrm{~d}_{3}\right)$

\subsubsection{Study of the Effect of Using the Type of the Day as One of the Dimensions}

In order to study the effect of adding the type of the day (weekend or working day) as on of the dimensions, we conducted the simulation to study the performance of the MSP-CACRR technique using two dimensions: the time intervals of the day and the type of the day. We refer to this technique as MSP-CACRR $\left(\mathrm{d}_{1}, \mathrm{~d}_{2}\right)$. The overall performance results are shown in Fig. 7.23, 7.24, and 7.25. As we can see from these results, using the type of the day as on of the dimensions enhances significantly the performance of the MSP-CACRR technique. This proves the hypothesis that the behavior of most of the mobile users will not be same during the weekend days. Most of the mobile users have different sequential patterns during the weekends and we able to extract these patterns using the MSP-CACRR technique. If 
we did not differentiate between the weekends and the working days, as in the MSP$\operatorname{CACRR}\left(\mathrm{d}_{1}\right)$, the prediction of the frequent sequences will not be accurate in most of the cases during the week end days. This will not force the network to make reservation for BW in the wrong cells which increase the number of dropped calls. Moreover, the wrong reservation of $\mathrm{BW}$ in many cells will decrease the available $\mathrm{BW}$ and may calls will be rejected. In this case, the network will not be able to utilize the provided BW effectively. Hence, it is very important to add the type of the day (weekend or working day) as on of the dimensions. This dimension will be used also in the next phase of comparisons.

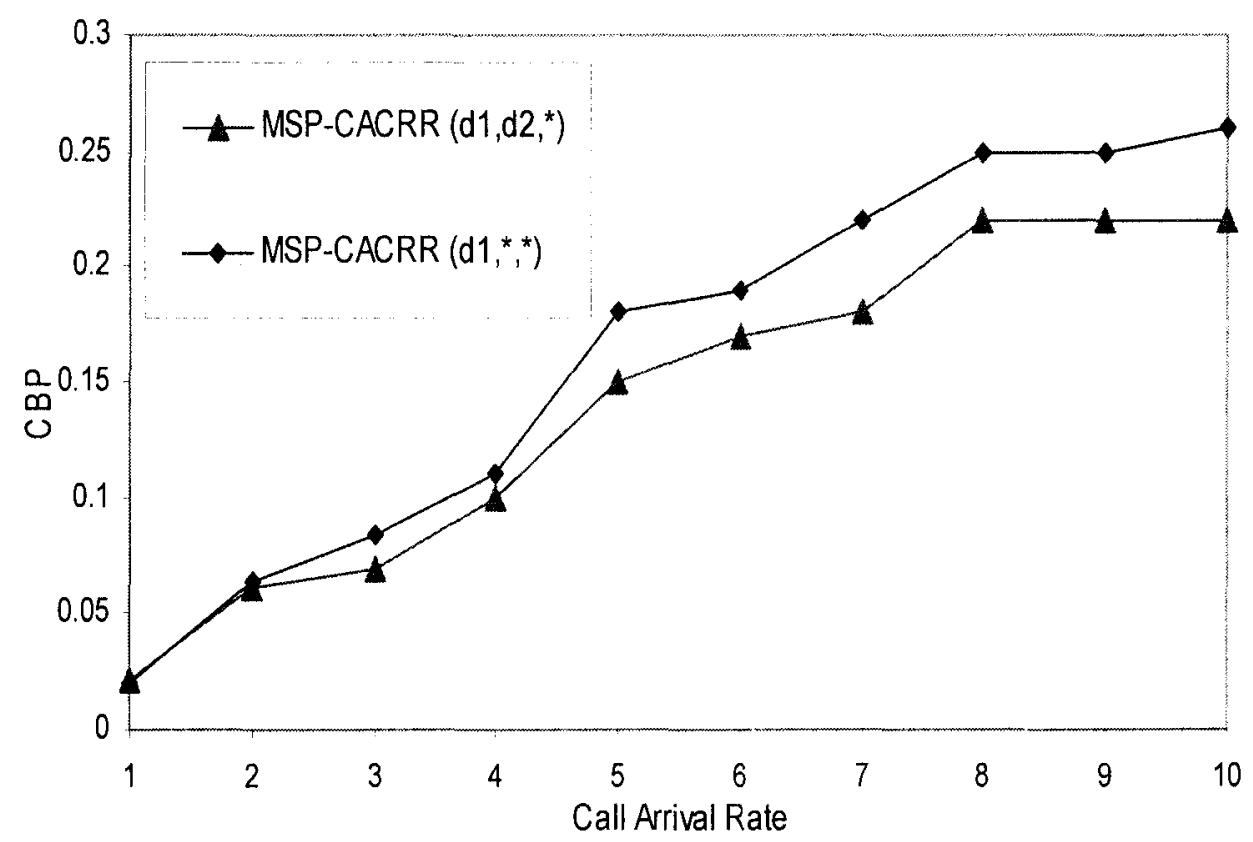

Fig. 7.23 Call blocking probability results comparing between MSP-CACRR $\left(\mathrm{d}_{1}\right)$ and MSP-CACRR $\left(\mathrm{d}_{1}, \mathrm{~d}_{2}\right)$ 


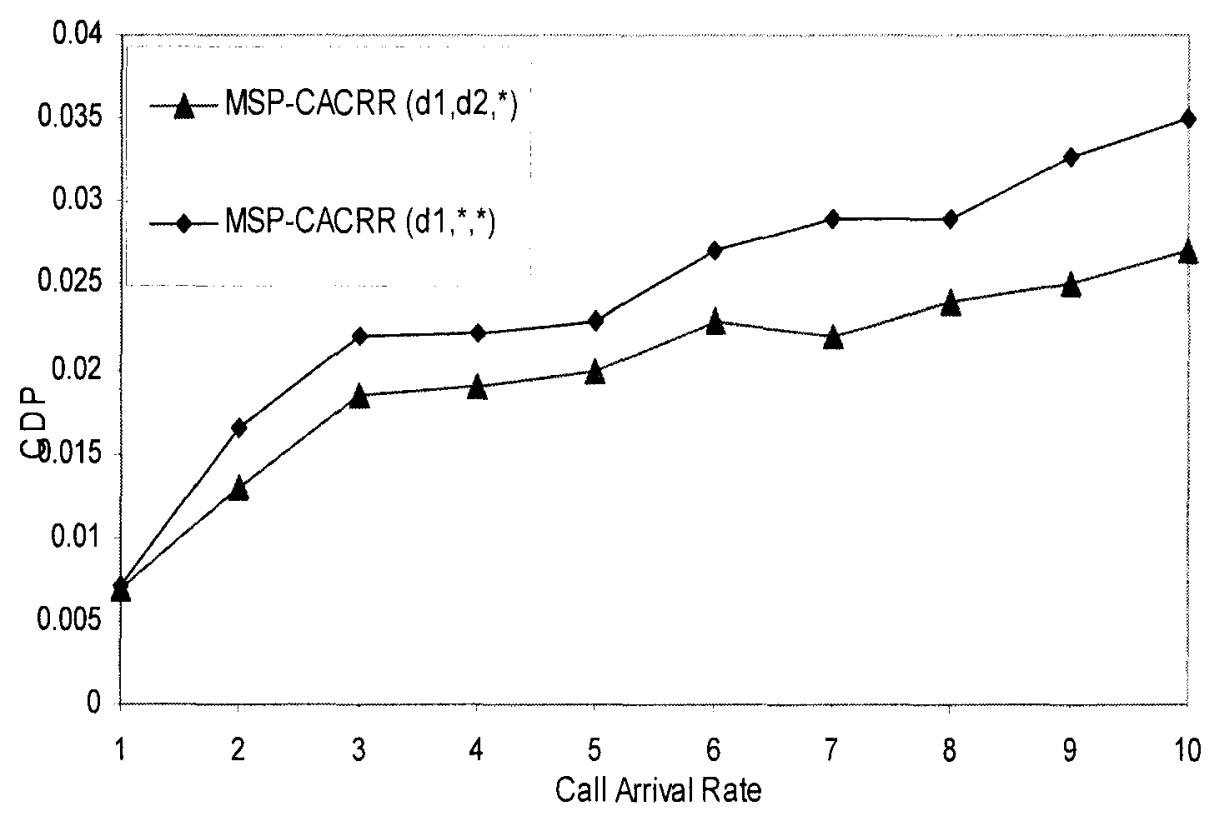

Fig. 7.24 Call dropping probability results comparing between MSP$\operatorname{CACRR}\left(\mathrm{d}_{1}\right)$ and MSP-CACRR $\left(\mathrm{d}_{1}, \mathrm{~d}_{2}\right)$

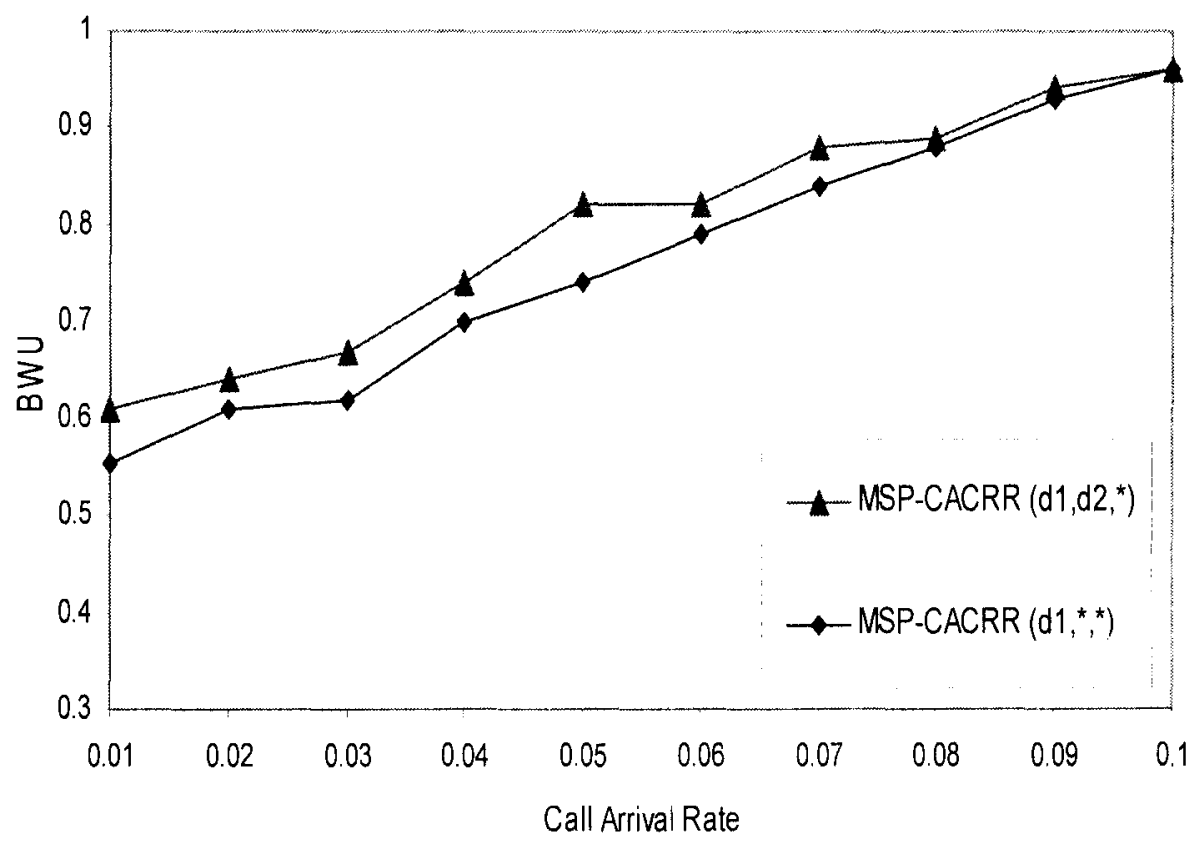

Fig. 7.25 Bandwidth utilization results comparing between MSP-CACRR $\left(\mathrm{d}_{1}\right)$ and MSP-CACRR $\left(\mathrm{d}_{1}, \mathrm{~d}_{2}\right)$ 


\subsubsection{Performance of MSP-CACRR Technique Compared to Other Techniques}

The simulation was also conducted to compare the performance of the MSPCACRR technique with PCAC-RR, PR-CAT4 and FR-CAT2. We used the two dimension version MSP-CACRR $\left(\mathrm{d}_{1}, \mathrm{~d}_{2}\right)$ which uses the time intervals and the type of the day as the basic dimensions. PCAC-RR is a predictive technique which utilizes the time interval and calculates the probabilities of finding the frequent full path. PRCAT4 is a predictive scheme and it takes into account the movement to the next cell from the previous cell through the current cell. In FR-CAT2 scheme, there is no prediction and a fixed portion of the total bandwidth is reserved for the handoff calls. The results are shown in Fig. 7.26, 7.27, and 7.28. As we can see from these results, MSP-CACRR $\left(d_{1}, d_{2}\right)$ has the overall best performance. From these figures, we can say that the predictive techniques have better performance in general compared to the non-predictive techniques. We focus here on the comparison between the MSP$\operatorname{CACRR}\left(\mathrm{d}_{1}, \mathrm{~d}_{2}\right)$ and PCAC-RR techniques since it has been shown before that PCAC-RR technique has a better performance compared to PR-CAT4 and FR-CAT2 techniques. We can see also that there is a significant enhancement in the performance of the MSP-CACRR $\left(\mathrm{d}_{1}, \mathrm{~d}_{2}\right)$ technique compared to the PCAC-RR. This is because of two main reasons. The first reason is that in the MSP-CACRR $\left(d_{1}, d_{2}\right)$ technique we use a new dimension to differentiate between the behavior of the user during the weekend and during the working days, while we use only the time intervals in the PCAC-RR. This significantly has an effect on the performance. The second reason is that in the MSP-CACRR $\left(\mathrm{d}_{1}, \mathrm{~d}_{2}\right)$ technique we used more efficient sequence mining technique (which is the MobilePrefixSpan technique) to extract the 
frequent sequences. This enables us to discover new frequent sequences that can not be discovered in the PCAC-RR.

To study the prediction quality of the MSP-CACRR $\left(\mathrm{d}_{1}, \mathrm{~d}_{2}\right)$ technique, we compared its performance with a Benchmark which will have the same $\mathrm{CAC}$ and $\mathrm{RR}$ technique with important assumption that the prediction is perfect. This means that for the Benchmark scheme, we know exactly what will be next path for each user and what will be the exact handoff time. The results of the Benchmark scheme are also shown in Fig. 7.26, 7.27, and 7.28.. As we can see from these figures, the average performance of MSP-CACRR $\left(\mathrm{d}_{1}, \mathrm{~d}_{2}\right)$ is about $85.27 \%$ of the average performance of the Benchmark. This ratio was about $70.65 \%$ in the PCAC-RR technique. This significant enhancement reflects that the MSP-CACRR $\left(\mathrm{d}_{1}, \mathrm{~d}_{2}\right)$ has a high prediction quality. The main reason for that is using the MobilePrefixSpan technique and the new additional dimension of the type of the day.

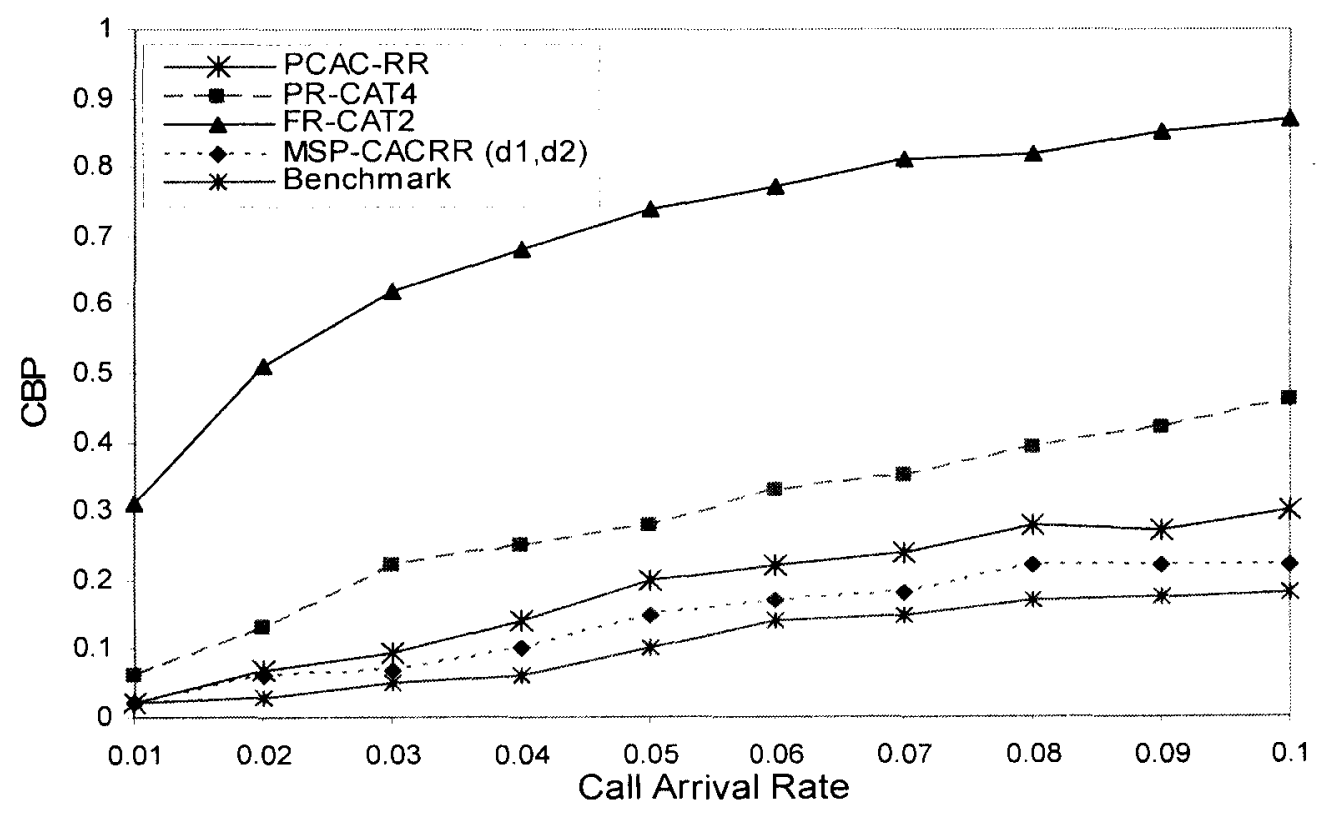

Fig. 7.26 Call blocking probability results comparing between MSP-CACRR $\left(\mathrm{d}_{1}\right.$, $\mathrm{d}_{2}$ ) and other techniques 


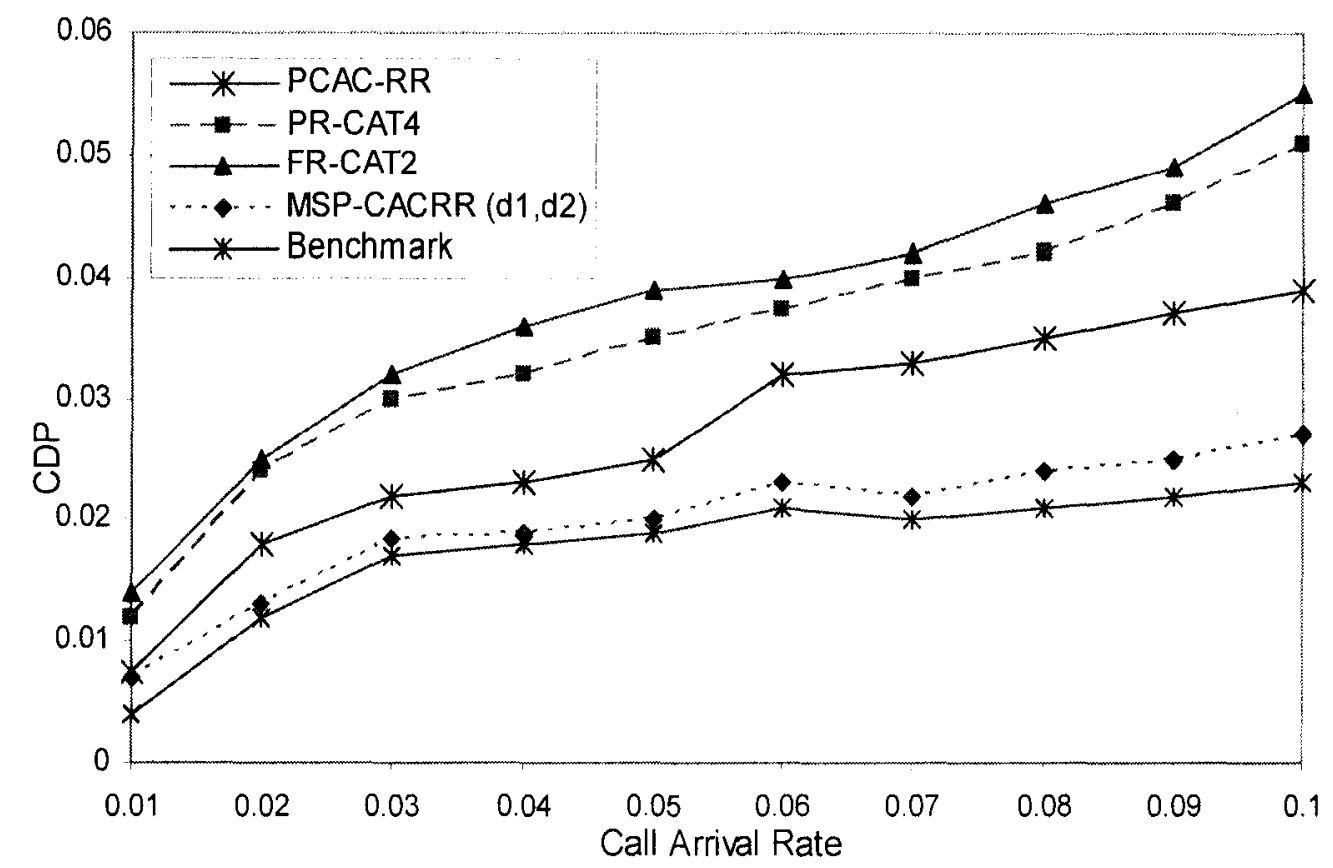

Fig. 7.27 Call dropping probability results comparing between MSP-CACRR $\left(\mathrm{d}_{1}\right.$, $\mathrm{d}_{2}$ ) and other techniques

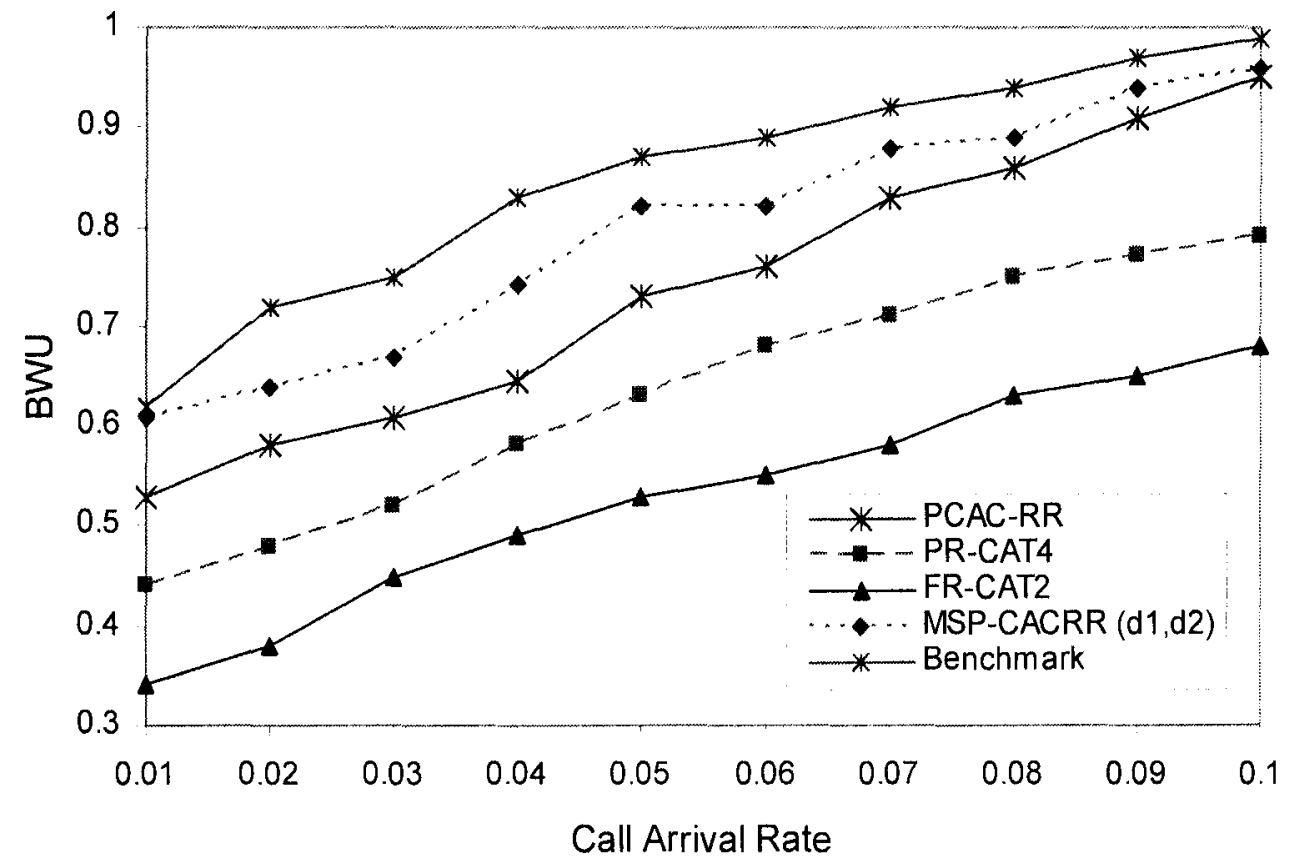

Fig. 7.28 Bandwidth utilization results comparing between MSP-CACRR $\left(d_{1}, d_{2}\right)$ and other techniques 


\subsection{Experimental Results for PCA-WHN}

The simulation was conducted to evaluate the performance of the PCA-WHN technique and compare its performance with the Benchmark technique. Benchmark technique is based on the same $\mathrm{CAC}$ and $\mathrm{RR}$ technique and it assumes that the prediction is perfect. For the Benchmark scheme, we know exactly what will be the next path for every user, and what will be the exact handoff time. This will enable us to compare the performance of the proposed PCA-WHN with the best approach.

We have conducted mainly two types of experiments. In the first one we have compared the performance of the PCA-WHN with the upper bound (the Benchmark approach). In the second type of experiments, we analyze the effect of using the WLAN in addition to the Cellular network. Therefore, we compare CAC and RR results with WLAN and without WLAN.

\subsubsection{Study of the Performance of PCA-WHN Technique}

In order to evaluate the performance of the PCA-WHN, we compared its performance with a Benchmark technique which will have the same CAC and RR technique with important assumption that the prediction is perfect. This means that for the Benchmark technique, we know exactly what will be next path of BS/AP (according to the type of the service) for each user and what will be the exact handoff time. The CAC algorithm for the Benchmark technique is shown in Appendix D. The results are shown in Fig. Fig. 7.29, 7.30, 7.31. Here we can see that the PCA-WHN have an average performance ratio $88.79 \%$ compared to the average performance of the benchmark technique. This means that using the PCA-WHN we can predict the 
mobility of the users with high accuracy comparing to the Benchmark. This is because using the predictive techniques allows us not only to predict the location and the time of handoff, but it also allows us to reserve the resources in the correct BS/AP according to the type of the services. Also it enables us to use the available resources in an efficient way to provide the service for many users with different service requirements.

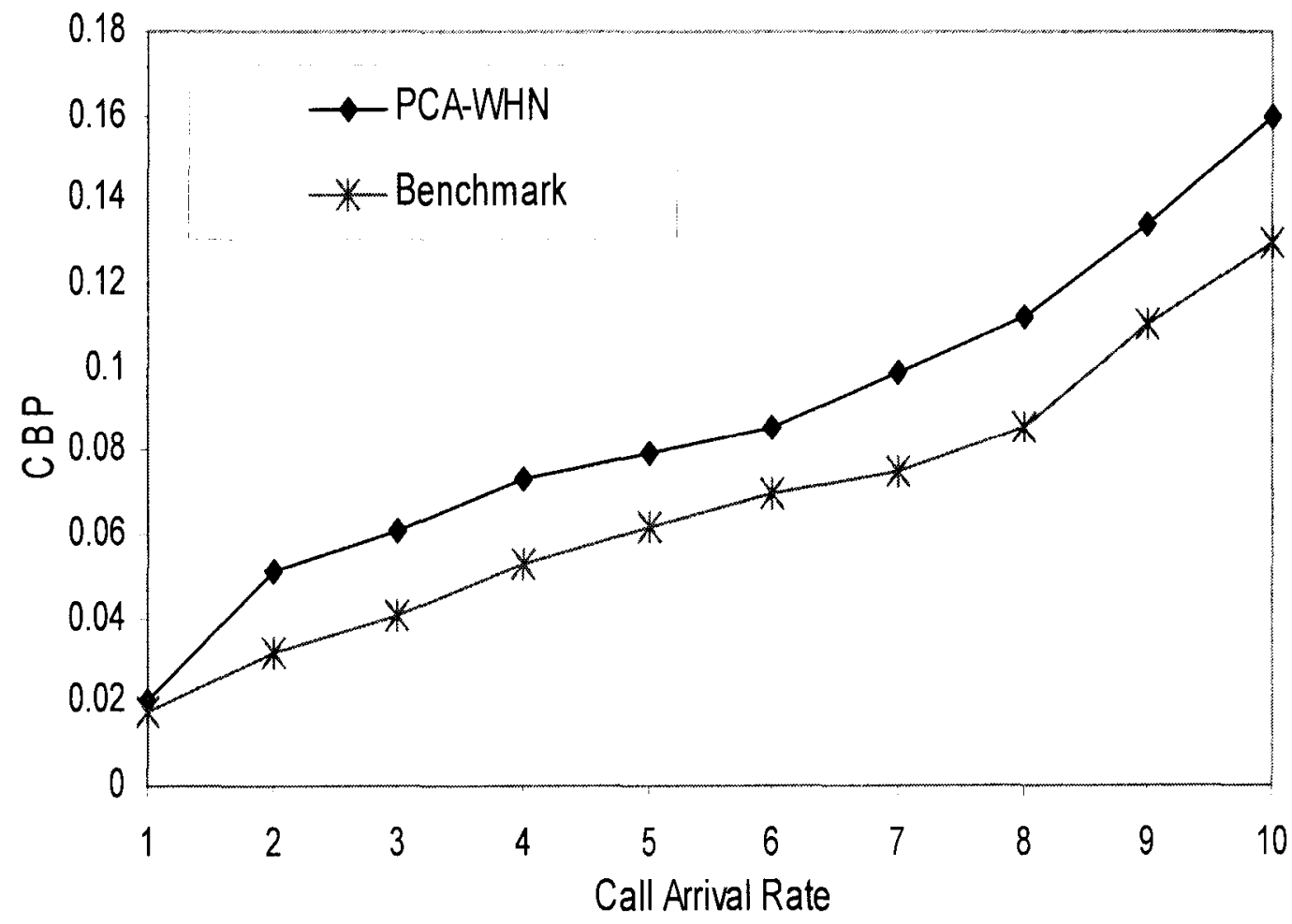

Fig. 7.29 Call blocking probability results comparing between PCAWHN and benchmark technique 


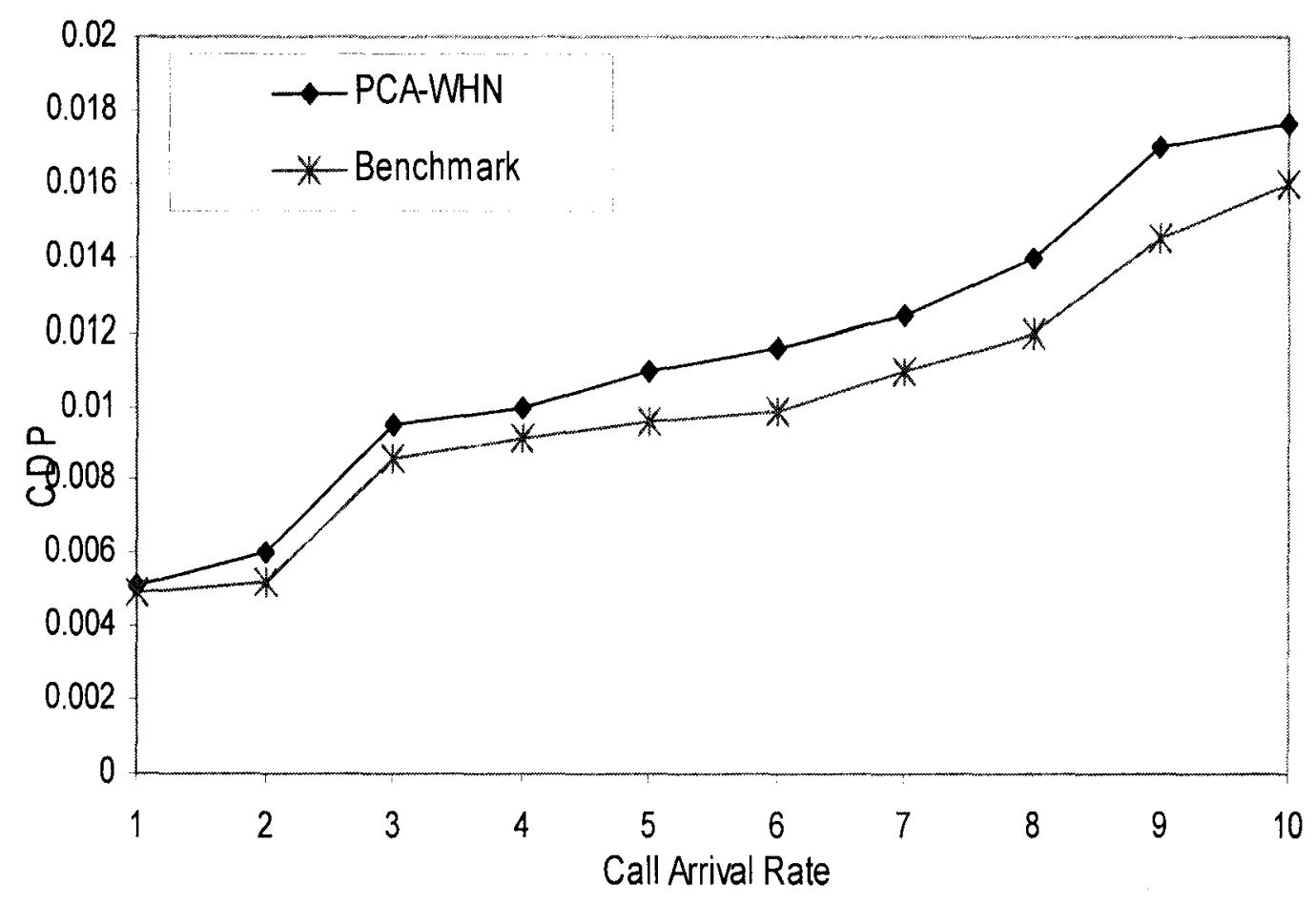

Fig. 7.30 Call blocking probability results comparing between PCAWHN and benchmark technique

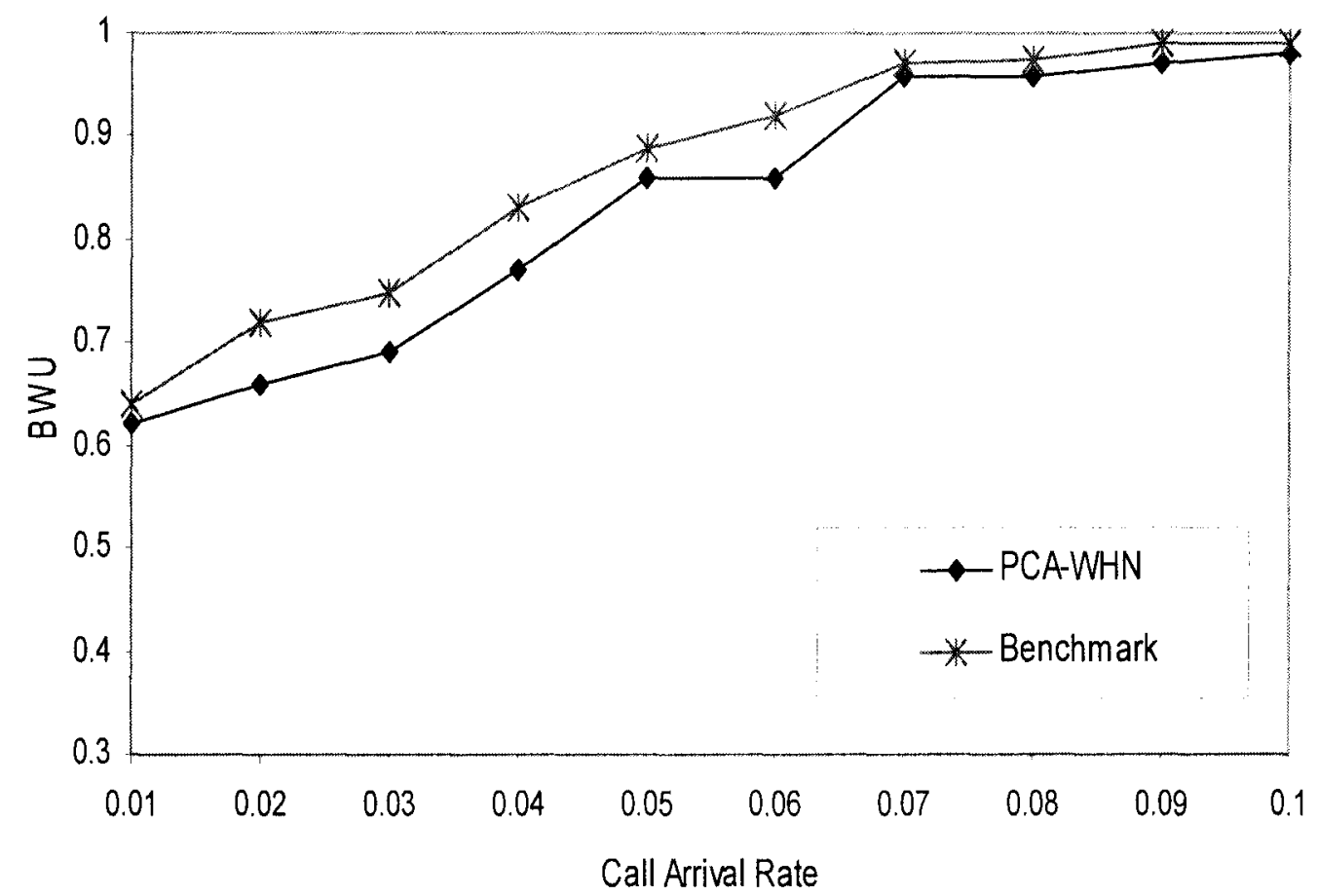

Fig. 7.31 Bandwidth utilization results comparing between PCA-WHN and benchmark technique 


\subsubsection{Study of the Impact of using WLAN}

In order to study the effectiveness of WHN, we conducted the simulation to study the performance of the network using cellular networks only and then using the heterogeneous networks of cellular network and WLAN. In both cases, we used the same PCA-WHN technique. The overall performances are shown in Fig. 7.32, 7.33, and 7.34. This shows the impact of using the WLAN in conjunction with cellular networks. We also compared the performance of the PCA-WHN with the PCAC-RR technique that we have introduced in for cellular networks. This will show also the impact of using the MobilePrefixSpan in prediction.

Using the WHN provided a better performance. We can see that the enhancement increases with the increasing call arrival rate. The WHN will be able to support more users because of the new available resources provided by WLAN. Also, this will help to accept more new calls and reduce the call blocking probability (CBP). But we can see that we still have also good bandwidth utilization because the network is able to make the correct reservation and predict the correct locations using the PCA-WHN technique. Also, the handoff dropping probability in this case is decreased because of the new available resources and because the ability of the network to make a correct reservation based on the required services. We can see from these results that using the MobilePrefixSpan technique enhances the overall performance especially the CDP measure as shown in Fig. 7.33. The main reason for this enhancement is that using MobilePrefixSpan technique enables us to discover new subsequences that could not be discovered using the PCAC-RR. 


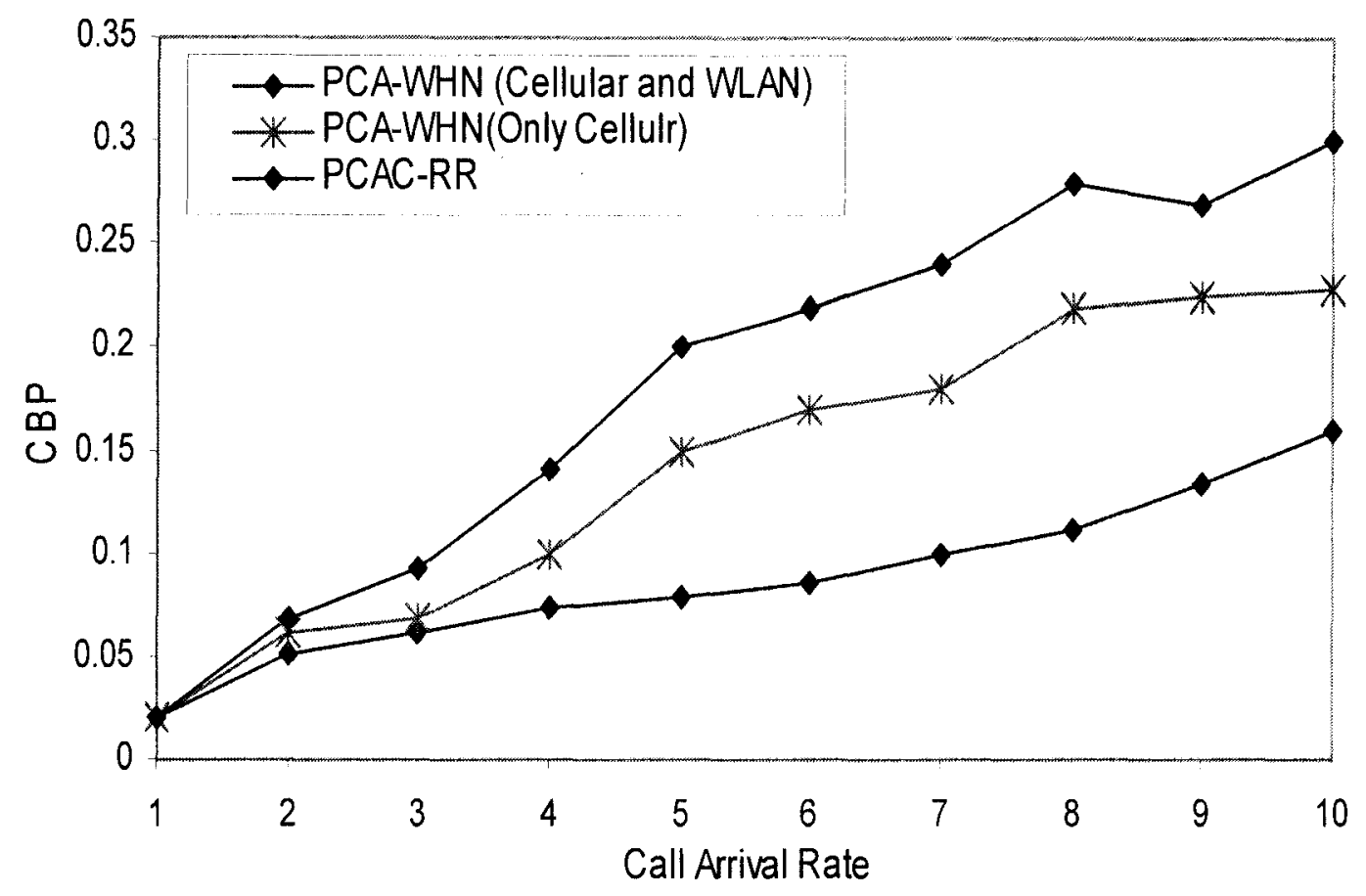

Fig. 7.32 Call blocking probability results comparing between cellular and heterogeneous networks

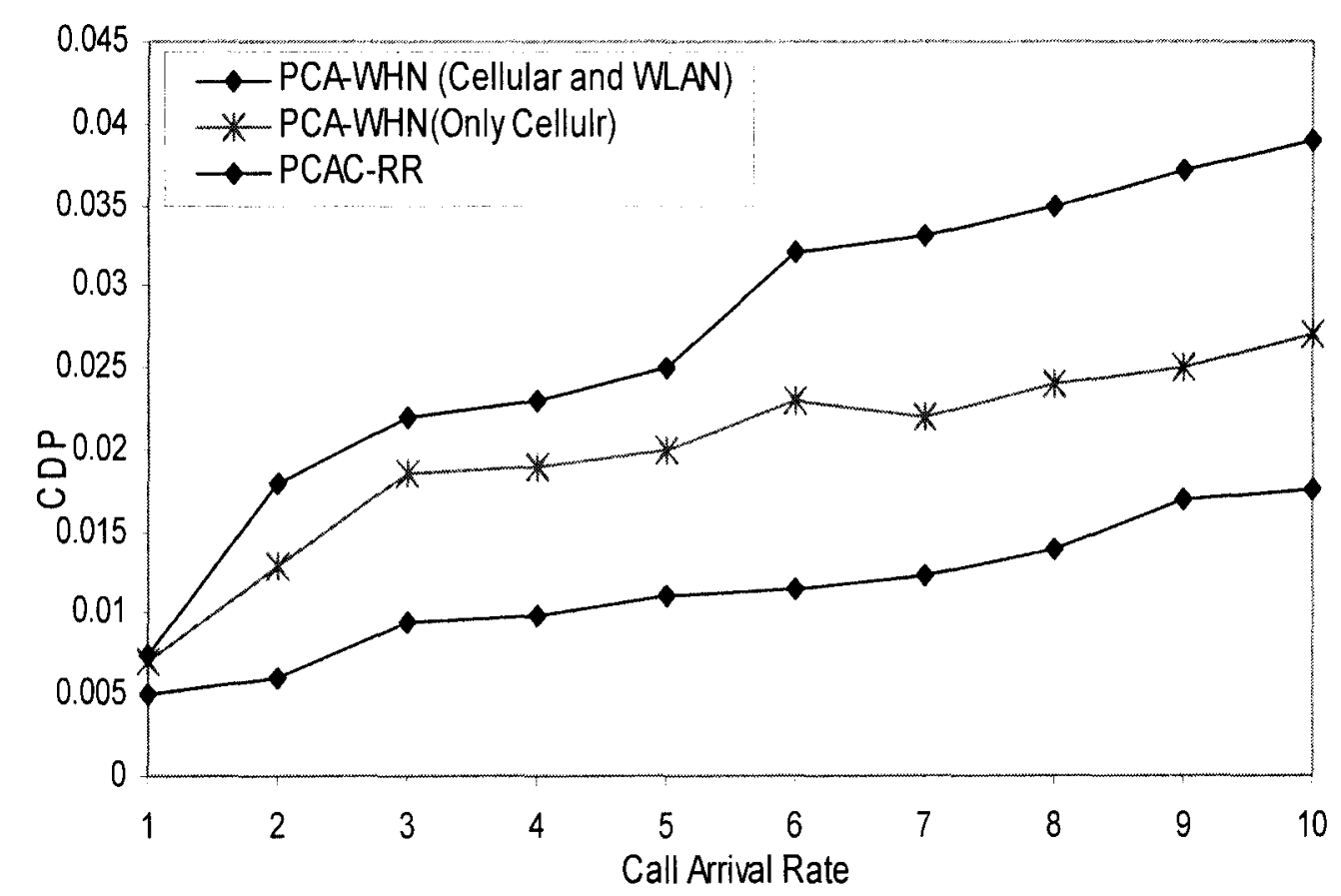

Fig. 7.33 Call dropping probability results comparing between cellular and heterogeneous networks 


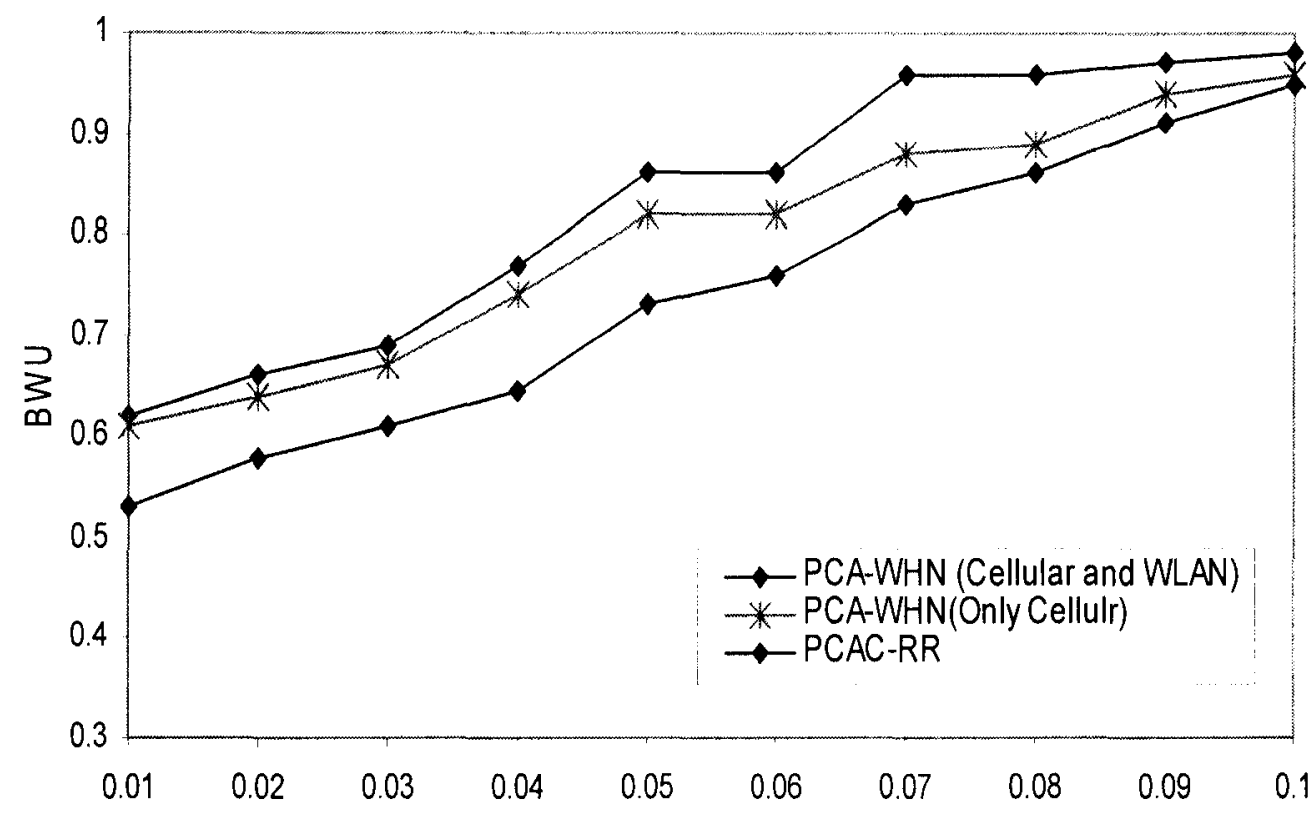

Call Arrival Rate

Fig. 7.34 Bandwidth utilization results comparing between cellular and heterogeneous networks 


\section{CHAPTER VIII}

\section{CONCLUSION AND FUTURE WORK}

In this chapter we summarize the contribution of this research and we give directions for future research.

\subsection{Research Contributions}

In this dissertation, the problems of call admission control and resource reservation were addressed in detail for two different architectures of wireless mobile networks: 1) cellular networks and 2) wireless heterogeneous networks (WHN). We summarize the contributions of this dissertation in the following points:

- A new predictive $\mathrm{CAC}$ and resource reservation and technique (PCAC-RR) that can be used for cellular networks has been proposed. The PCAC-RR technique is based on the generation of mobility profiles. These mobility profiles are used by the PCAC-RR technique to predict the future path of the mobile user.

- The performance of the PCAC-RR technique was studied to see the effects of:

i. Different types of mobility profiles

ii. Different number of time intervals

iii. The repeated sequences.

The simulation results proved that using a combination of local and global mobility profiles has enhanced the results significantly. Using global profiles allowed 
us to enhance the prediction in the case of low prediction accuracy for the local profiles. The frequent paths in both local and global levels are utilized to predict the next movements and the time at which the handoff will occur. Also, it has been proven that the number of time intervals affects the performance, and it has to be selected carefully according to the mobile environment to enhance the overall performance. In our study, we found that four time intervals is a suitable selection. We proved that using the repeated sequences was a very important factor to predict the handoff time.

- The proposed technique has the advantage of using the time as one of the important factors in extraction of the frequent paths and also in prediction of the handoff time. This enhanced the prediction accuracy and the utilization of the bandwidth.

- To compare PCAC-RR and other techniques, the simulation was also performed for the PCAC-RR technique as well as FR-CAT2 and FR-CAT4 techniques. The simulation results in terms of $\mathrm{CBP}, \mathrm{CDP}$ and $\mathrm{BWU}$ showed that the proposed PRR-PCAC technique has a significantly better performance compared to the other two techniques (in average $27 \%$ of enhancement compared to the predictive PR-CAT4 technique and more that $50 \%$ compared to the FR-CAT2). Prediction of the next cells is an important factor in solving the problems of CAC and resource reservation, and they are related directly to the mobility of the users in the mobile communications.

- We have developed the MobilePrefixSpan technique to extract the frequent sequences effectively from the multidimensional collected records. 
MobilePrefixSpan is a modified version of the well-known sequence mining technique Prefix-Span and its modified version for multidimensional sequences.

- We have proposed a second technique for CAC and RR in cellular networks called MSP-CACRR, which is based on multidimensional sequence mining. This technique uses our efficient sequential pattern mining technique, MobilePrefixSpan. Experimental results show that there are two main dimensions to be used in the next generation of cellular networks to enhance the CAC and RR performance. These two dimensions are the time intervals of the day and the type of the day (weekend or working day). It has bee shown that there is no need to use the service type as one of the dimensions for this type of mobile networks (cellular networks). Also, the simulation results in terms of CBP, CDP and BWU show that there is a significant improvement in the overall performance of the MSP-CACRR compared to other predictive techniques. Comparing MSP-CACRR with a Benchmark technique (which has a perfect prediction), has shown that the quality of prediction in the MSP-CACRR technique using the MobilePrefixSpan enables us to reach $85.27 \%$ of the average performance of the Benchmark technique.

- We have also proposed and evaluated a new CAC and RR technique (called PCAWHN) that we have designed for wireless heterogeneous networks that integrates cellular networks and WLANs. PCA-WHN provides effective connection management and handover between heterogeneous systems and it takes into account the different characteristics of the cellular networks and the WLANs. PCA-WHN uses MobilePrefixSpan to generate the mobility patterns. These 
patterns are generated after collecting the spatial, the temporal, and the usage information of the mobile users. This enables the network to predict the location of the mobile users in an accurate way and to enhance the resource reservation process. Experimental results show that there is a need to use the WLAN in addition to the cellular networks in the heterogeneous networks. This enables the network to accommodate more users and enhance the overall performance especially with increasing the loads on the network. Also experimental results showed that using PCA-WHN enabled us to reach $88.79 \%$ of the average performance of the Benchmark technique, this is due to the enhanced prediction of the PCA-WHN.

\subsection{Future Research Directions}

The future work for researchers in this area includes enhancing the proposed techniques; and applying the mobility prediction approach to other problems in mobile networks.

To enhance the predictive $\mathrm{CAC}$ and $\mathrm{RR}$ techniques we can use the incremental updating to update the mobility profiles instead of using the direct updating after a period of time. This will enhance prediction accuracy and the overall performance of the $\mathrm{CAC}$ and RR techniques.

The mobility prediction approach can be applied to solve other problems in the mobile networks such as multicasting, broadcasting, and routing problems. This approach can be applied to solve these problems in different structures of mobile networks. Also, the proposed PCA-WHN technique can be applied to other structures of wireless heterogeneous networks where we have other different types of networks 
such as mobile Ad-hoc networks and cellular networks. Finally, these predictive techniques can be applied to wireless mesh networks. 


\section{REFERENCES}

[1] Hai Jiang; Weihua Zhuang, "Quality-of-service provisioning in future 4G CDMA cellular networks", IEEE Wireless Communications, pp. 48- 54, Vol.11, Issue: 2, Sept 2004.

[2] Akyildiz, I. F., McNair. J., Ho, J. S. M., Uzunalioglu, H., and Wang, W., "Mobility Management in Next-Generation Wireless Systems", Proc. of the IEEE, Vol. 87, No. 8, pp. 1347-1384, August 1999.

[3] Amitava Mukherjee, Somprakash Bandyopadhyay and Debashis Saha: Location Management and Routing in Mobile Wireless Networks, Artech House Publishers, May 2003.

[4] P. Nicopolitidis, M. S. Obaidat, G. I. Papadimitriou, A. S. Pomportsis: Wireless Networks, Wiley, 2003.

[5] Jens Zander, Seong-Lyun Kim, Magnus Almgren, Olav Queseth: Radio Resource Management for Wireless Networks, Artech House Publishers, April, 2001.

[6] Kadi, M. E., Olariu, S. and Wahab, H. A., "A Rate-based Borrowing Schemes for QoS Provisioning in Multimedia Wireless Networks", IEEE Transactions on Parallel and Distributed Systems, Vol. 13, No. 2, pp. 156-166, February 2002. 
[7] Y. Fang and Y. Zhang, "Call admission control schemes and performance analysis in wireless mobile networks," IEEE Transactions on Vehicular Technology, Vol.51, No.2, 371-382, March 2002.

[8] Ganguly, S. and Nath, B., "An Implicit QoS Provisioning Strategy in Multimedia Cellular Network", IEEE Wireless Communications and Networking Conference (WCNC2002), Vol. 1, pp. 301-306, 2002.

[9] C. Chang, C. J. Chang, and K. R. Lo, "Analysis of a hierarchical cellular system with reneging and dropping for waiting new calls and handoff calls," IEEE Trans. Veh. Technol., Vol. 48, No. 4, pp. 1080-1091, 1999.

[10] D. E. Everitt, "Traffic engineering of the radio interface for cellular mobile networks," Proc. Of the IEEE, Vol. 82, No. 9, pp. 1371-1382, 1994.

[11] M. D. Kulavaratharasah and A. H. Aghvami, "Teletraffic performance evaluation of microcellular personal communication networks (PCN's) with prioritized handoff procedures," IEEE Trans. Veh. Technol., Vol. 48, No. 1, pp. 137-152, 1999.

[12] V. K. N. Lau and S. V. Maric, "Mobility of queued call requests of a new callqueuing technique for cellular systems," IEEE Trans. Veh. Technol., Vol. 47, No. 2, pp. $480-488,1998$.

[13] Chen, H., Kumar, S. and Kuo, C. C. J., "Dynamic Call Admission Control Scheme for QoS Priority Handoff in Multimedia Cellular Systems", IEEE Wireless Communications and Networking Conference (WCNC), Vol. 1 , pp. 17-21, March 2002. 
[14] Levine, D. A., Akyildiz, I. F. and Naghshineh, M., "A Resource Estimation and Call Admission Algorithm for Wireless Multimedia Networks using the Shadow Cluster Concepts", IEEE/ACM Transactions on Networking, Vol. 5, No. 1, pp. 1-12, February 1997.

[15] Islam, M. M., Murshed, M.and Dooley, L. S., "A Direction Based Bandwidth Reservation Scheme for Call Admission Control", International Conference on Computer and Information Technology (ICCIT2002), pp. 345-349, Dec 27-28, 2002, Dhaka, Bangladesh.

[16] Kwon, T., Choi, Y., Bisdikian, C. and Naghshineh, M., "Measurement-based Call Admission Control for Adaptive Multimedia in Wireless/Mobile Networks", IEEE Wireless Communications and Networking Conference, 1999 (WCNC 1999), Vol. 2, pp. 540-544, 1999.

[17] Akyildiz, I.F.; Jiang Xie; Mohanty, S.; "A survey of mobility management in next-generation all-IP-based wireless systems", IEEE Wireless Communications, Vol. 11 , Issue 4 , pp. 16-28, Aug. 2004.

[18] Quintero, A.; Garcia, O., "A profile-based strategy for managing user mobility in third-generation mobile systems", IEEE Communications Magazine, Vo.1.42 Issue 9, pp. $134-139$, Sept 2004.

[19] Xiao, Y.; Pan, Y.; Li, J.;"Design and analysis of location management for 3G cellular networks", IEEE Transactions on Parallel and Distributed Systems, , Vol. 15 , Issue 4 , pp. $339-349$, April 2004. 
[20] Il Han; Dong-Ho Cho; "Group location management for mobile subscribers on transportation systems in mobile communication networks", IEEE Transactions on Vehicular Technology, Vol. 53 , Issue 1, pp. 181 - 191, Jan. 2004.

[21] Islam, M.M.; Murshed, M.; "Novel velocity and call duration support for QoS provision in mobile wireless networks", IEEE Wireless Communications, Vol. 11, Issue 5 , pp. $22-30$, Oct. 2004.

[22] Akyildiz, I.F.; Wang, W.; "The Predictive User Mobility Profile Framework for Wireless Multimedia Networks", IEEE/ACM Transactions on Networking, Vol. 12, Issue 6, pp. 1021 - 1035, Dec. 2004.

[23] Yilmaz, N.; Ergul, R.; "Resource management in cellular communication networks with subscriber profile prediction", The Ninth International Conference on Communications Systems (ICCS 2004), pp. 180- 184, Sept. 2004.

[24] Pandey, V.; Ghosal, D.; Mukherjee, B.; "Exploiting user profiles to support differentiated services in next-generation wireless networks", IEEE Network, Vol. 18 , Issue 5, pp. $40-48$, Sept.-Oct. 2004.

[25] Ng, C.K.; Chan, H.W.;" Enhanced Distance-Based Location Management of Mobile Communication Systems Using a Cell Coordinates Approach", IEEE Transactions on Mobile Computing, Vol. 04 , Issue 1, pp. 41 - 55 , Jan.-Feb. 2005. 
[26] Mohammad Mahfuzul Islam, Manzur Murshed and Laurence S. Dooley, "New Mobility Based Call Admission Control with On-Demand Borrowing Scheme for Qos Provisioning", International Conference on Information Technology: Coding and Computing [Computers and Communications], ITCC 2003, pp. 263-267, April 2003.

[27] F. Yu and V.C.M. Leung, "Mobility-based predictive call admission control and bandwidth reservation in wireless cellular networks", Computer Networks, Vol. 38, No. 5, pp. 577-589, April 2002.

[28] Chang Ho Choi, Myung Il Kim, Sung-Jo Kim, "Call Admission Control Using the Moving Pattern of Mobile User for Mobile Multimedia Networks", 27th Annual IEEE Conference on Local Computer Networks, pp. 431-440, Nov. 2002.

[29] J. Hou and Y. Fang, "Mobility-based call admission control schemes for wireless mobile networks," Wireless Communications and Mobile Computing, Vol.1, No.3, pp. 269-282, July-September 2001.

[30] Jon M. Peha, Arak Sutivong, "Admission control algorithms for cellular systems", Wireless Networks, Vol. 7, Issue 2, pp. 117 - 125, March/April 2001.

[31] Wee-Seng Soh and Hyong S. Kim, "QoS Provisioning in Cellular Networks Based on Mobility Prediction Techniques", IEEE Communications Magazine, pp. 86-92, Jan. 2003. 
[32] Wee-Seng Soh and Hyong S. Kim, "Dynamic Bandwidth Reservation in Cellular Networks Using Road Topology Based Mobility Predictions", Proceeding of IEEE INFOCOM 2004, Vol. 4, pp. 2766-2777, Mar. 2004.

[33] Duan-Shin Lee and Yun-Hsiang Hsueh, "A Bandwidth Reservation Scheme Based on Road Information for the Next Generation Cellular Networks", IEEE Trans. on Vehicular Technology, Vol. 53, No. 1, pp. 243--252, January 2004

[34] S. Choi and K. G. Kin, "Predictive and adaptive bandwidth reservation for handoffs in QoS-sensitive cellular networks," in Proc. ACM SIGCOMM'98, Vancouver, BC, Canada, pp. 155-166, Sept. 1998,

[35] Fei Yu; Wong, V.W.S.: Leung, V.C.M.., "Performance enhancements of combining QoS provisioning and location management in wireless cellular networks", in the Proc. of IEEE Global Telecommunications Conference, (GLOBECOM '02), Vol. 1 , pp. 956 - 960, Nov. 2002.

[36] Fei Yu; Leung, V.C.M., "A framework of combining mobility management and connection admission control in wireless cellular networks", in the Proc. of IEEE International Conference on Communications, (ICC 2001), Vol.7 , pp.11-14, June 2001.

[37] S. Rashad, M. Kantardzic, and A. Kumar, "A Data Mining Approach for Call Admission Control and Resource Reservation in Wireless Mobile Networks". In P. Perner (Ed.), Advances in Data Mining - Applications in Image Mining, Medicine and Biotechnology, Management and Environmental Control, and 
Telecommunications, LNCS: LNAI Vol. 3275, pp. 134-143, Springer Verlage, November 2004.

[38] Sherif Rashad, Mehmed Kantardzic, Anup Kumar, "PCAC-RR: A Predictive Call Admission Control and Resource Reservation for Wireless Mobile Networks", Proceedings of the First IEEE and IFIP International Conference on Wireless and Optical Communications Networks (WOCN2004), pp. 295298, Muscat, Oman, 7-9 June 2004.

[39] Sherif Rashad, Mehmed Kantardzic, Anup Kumar, "Mobile Data Mining for Radio Resource Management in Wireless Mobile Networks", in the proceeding of IEEE and ACM International Conference on Machine Learning and Applications (ICMLA'04), pp.85-92, Dec. 2004.

[40] Mehmed Kantardzic, Data Mining: Concepts, Models, Methods, and Algorithms. Piscataway, IEEE Press, 2003.

[41] R. Agrawal and R. Srikant, "Mining Sequential Patterns", Proceedings of IEEE Conference on Data Engineering, pp.3-14, 1995.

[42] M. Zaki, "Efficient enumeration of frequent sequences", in Proc. CIKM: ACMCIKM Int. Conf. Information KnowledgeManagement, 1998, [Online]. Available at: citeseer.nj.nec.com/zaki98efficient.html.

[43] Ding-Ying Chiu; Yi-Hung Wu; Chen, A.L.P., An Efficient Algorithm For Mining Frequent Sequences By A New Strategy Without Support Counting, 20th International Conference on Data Engineering, pp. 375 - 386, 2004. 
[44] Jian Pei, Jiawei Han, Behzad Mortazavi-Asl, Jianyong Wang, Helen Pinto, Qiming Chen, Umeshwar Dayal, Meichun Hsu, "Mining Sequential Patterns by Pattern-Growth: The PrefixSpan Approach", IEEE Transaction on. Knowledge and Data Engineering, vpl. 16, no. 11, pp. 1424-1440, November 2004.

[45] J. Pei, J. Han, B. Mortazavi-Asl, H. Pinto, Q. Chen, U. Dayal, and M.-C. Hsu, "PrefixSpan: Mining Sequential Patterns Efficiently by Prefix-Projected Pattern Growth," Proc. 2001 Int'l Conf. Data Eng. (ICDE '01), pp. 215-224, Apr. 2001.

[46] Helen Pinto, Jiawei Han, Jian Pei, Ke Wang, Qiming Chen, Umeshwar Dayal, "Multi-dimensional Sequential Pattern Mining", Proceedings of the 2001 ACM CIKM International Conference on Information and Knowledge Management, Atlanta, Georgia, pp. 81-88, November 2001.

[47] Chung-Ching Yu, Yen-Liang Chen: Mining Sequential Patterns from Multidimensional Sequence Data. IEEE Transaction on. Knowledge and Data Engineering, vol. 17, no. 1, pp. 136-140, January 2005.

[48] N. Eagle, "Machine Perception and Learning of Complex Social Systems", Ph.D. Thesis, Massachusetts Institute of Technology, June 2005.

[49] Narumi Umeda, Toru Otsu, and Tatsuro Masamura, "Overview of the Fourthgeneration Mobile Communication System", NTT Technical Review, Vol.2 No.9, pp. 12-17, September 2004. 
[50] Taha, A.-E.M.; Hassanein, H.S.; Mouftah, H.T., "On robust allocation policies in wireless heterogeneous", First International Conference on networks Quality of Service in Heterogeneous Wired/Wireless Networks, QSHINE 2004, pp. $198-205,204$.

[51] J. Manner, L. Burness, E. Hepworth, A. Lopez and E. Mitjana, "Provision of QoS in Heterogeneous Wireless IP Access Networks," IEEE International Symposium on Personal, Indoor and Mobile Radio Communications, Volume 2. pp. 530- 534, September 2002.

[52] L. Dell'Uomo and E. Scarrone, "An All-IP solution for QoS Mobility Management and AAA in the 4G Mobile Networks," International Symposium on Wireless Personal Multimedia Communications, Volume 2, pp. 591-595, October 2002.

[53] M. Inoue, G. Wu, K. Mahmud, H. Murakami and M. Hasegawa, "Development of MIRAI System for Heterogeneous Wireless Networks," IEEE International Symposium on Personal, Indoor and Mobile Radio Communications, Volume 1. pp. 69-73, September 2002.

[54] L. Morand and S. Tessir, "Global Mobility Approach with Mobile IP in 'All IP' Networks," IEEE International Conference on Communications, Volume 4, pp. 2075-2079, May 2002.

[55] Cavalcanti, D.; Agrawal, D.; Cordeiro, C.; Bin Xie; Kumar, A.; "Issues in integrating cellular networks, wlans, and manets: a futuristic heterogeneous 
wireless network", IEEE Wireless Communications Magazine,Volume 12, Issue 3, pp. 30 - 41 , June 2005.

[56] F. Akyildiz, J. Xie, and S. Mohanty, "A Survey on Mobility Management in Next Generation All-IP Based Wireless Systems," IEEE Wireless Communications Magazine, vol. 11, no. 4, pp. 16-28, Aug. 2004.

[57] Hyosoon Park, Sunghoon Yoon, Taehyoun Kim, Jungshin Park, Misun Do, and Jaiyong Lee, "Vertical Handoff Procedure And Algorithm Between IEEE802.11 WLAN And CDMA Cellular Network," CIC2002, Vol. 11, pp217-221, Seoul, Korea,2002.

[58] Y. Cheng, H. Jiang, W. Zhuang, Z. Niu, and C. Lin, "Efficient resource allocation for China's $3 \mathrm{G} / 4 \mathrm{G}$ wireless networks", IEEE Communications Magazine, vol. 43, no. 1, pp. 76-83, Jan. 2005.

[59] Kasera, S.K.; Ramjee, R.; Thuel, S.R.; Xin Wang, "Congestion Control Policies for IP-Based CDMA Radio Access Networks", IEEE Transactions on Mobile Computing, , Vol.4, Iss.4, pp.349- 362, July-Aug. 2005.

[60] Karabudak, D.; Chih-Cheng Hung; Bing, B., "An intelligent admission control scheme for next generation wireless systems using distributed genetic algorithms", 2004 IEEE Wireless Communications and Networking Conference, WCNC.2004., Vol.3, pp. 1748- 1753, March 2004.

[61] Kelvin L. Dias, Stênio F. L. Fernandes, Djamel F. H. Sadok, "Predictive call admission control for all-IP wireless and mobile networks", the 2003 
IFIP/ACM Latin America conference on Towards a Latin American agenda for network research, pp. $131-139,2003$.

[62] L. Bow and W. Leroy, "Toward an all-IP based UMTS System Architecture," IEEE Network, 15(1), Jan-Feb 2001, pp.36-45

[63] R. Berezdivin et al., "Next-Generation Wireless Communications Concepts and Technologies," IEEE Comm. Mag., 40(3), Mar 2002, pp. 108-116.

[64] R. Chakravorty et al., "Performance Issues with Vertical Handovers Experiences from GPRS Cellular and WLAN Hot-Spots Integration," Proc. 2nd IEEE Conf. Pervasive Comp. and Commun., Mar. 2004.

[65] H. Zhu, M. Li, I. Chlamtac and B. Prabhakaran, "A Survey of Quality of Service in IEEE 802.11 Networks," IEEE Wireless Communications Magazine, Vol.11, Iss.4, pp. 6-14, Aug. 2004.

[66] N. Montavont, T. Noel, and M. Kassi-Lahloy, "Description and Evaluation of Mobile IPv6 for Multiple Interfaces," Proc. IEEE Wireless Commun. and Net. Conf.,Atlanta, GA, Mar. 2004.

[67] Al Agha, K.; Al-Meshhadany, T.; Perato, L.;" Resource allocation based on handoff prediction in WCDMA", Proceedings of IEEE 56th Vehicular Technology Conference, VTC, Vol.1, pp. 127 -131, 24-28 Sept. 2002.

[68] Lam, D.; Cox, D.C.; Widom, J.," Teletraffic modeling for personal communications services", IEEE Communications Magazine, Vol. 35 , Issue: 2, pp. 79 - 87, Feb. 1997. 
[69] Yi-Bing Lin, A. NoerPel, and D. Harasty, "A Non-Blocking Channel Assignment Strategy for Hand-Offs," IEEE ICUPC94, pp. 558 - 562, September 1994.

[70] Sherif Rashad, Mehmed Kantardzic, and Anup Kumar, "User Mobility Oriented Predictive Call Admission Control and Resource Reservation for Next-Generation Mobile Networks", Journal of Parallel and Distributed Computing (JPDC), Elsevier, Volume 66, Issue 7, pp. 971-988, July 2006.

[71] Sherif Rashad, Mehmed Kantardzic, and Anup Kumar, "Time Component Analysis of Enhanced Predictive Call Admission Control in Wireless Cellular Networks", GESTS International Transaction on Computer Science and Engineering, Vol. 7, No.1, pp.75-86, May 2005. 


\section{APPENDIX A}

A.1 The algorithm of finding frequent paths for the local profile

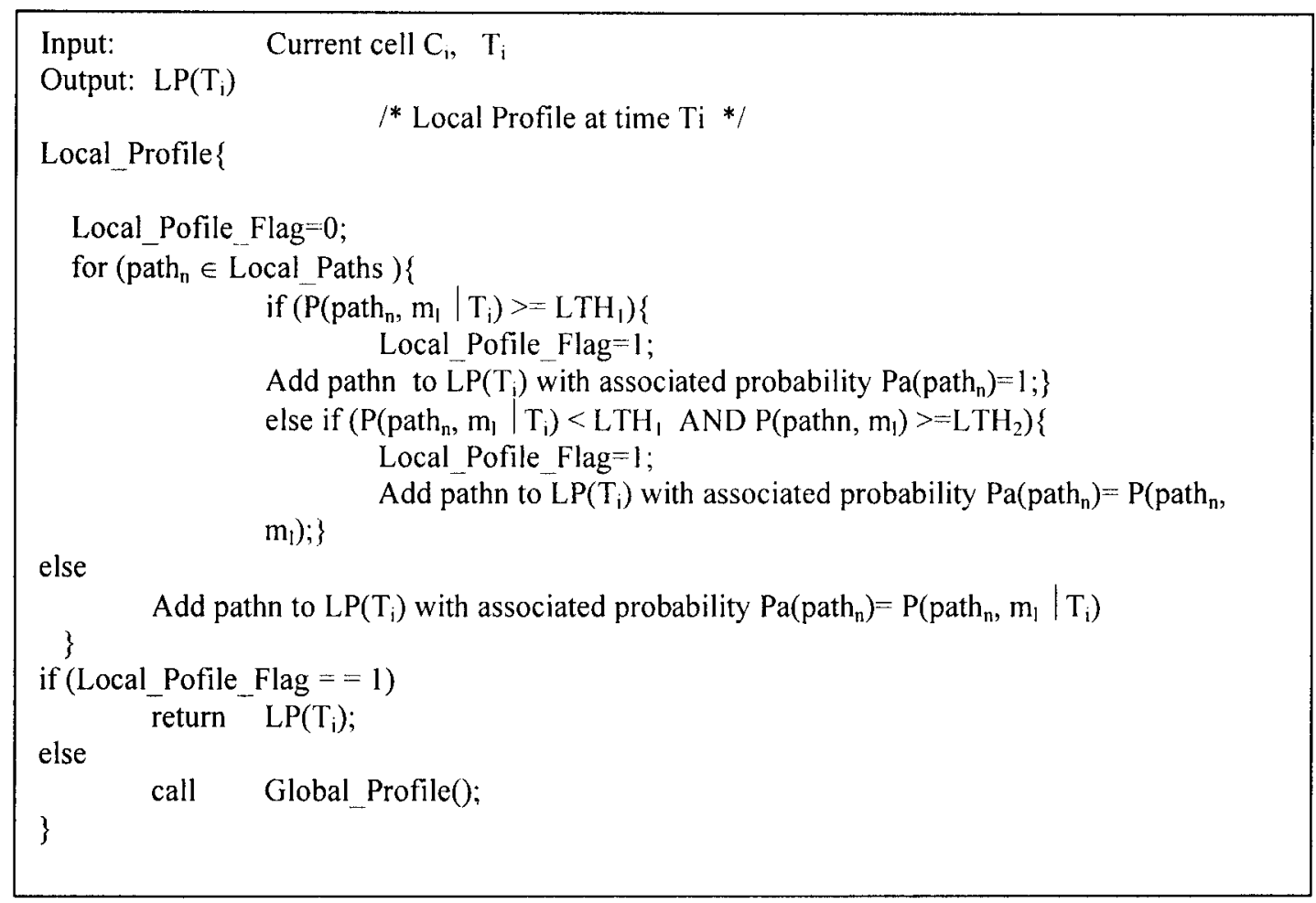

A.2 The algorithm of finding frequent paths for the global profiles

\begin{tabular}{|c|c|}
\hline $\begin{array}{l}\text { Input: } \\
\text { Output: GP }\left(T_{i}\right) \\
\text { Global_Profile } \\
\quad \text { for (path } \\
\end{array}$ & 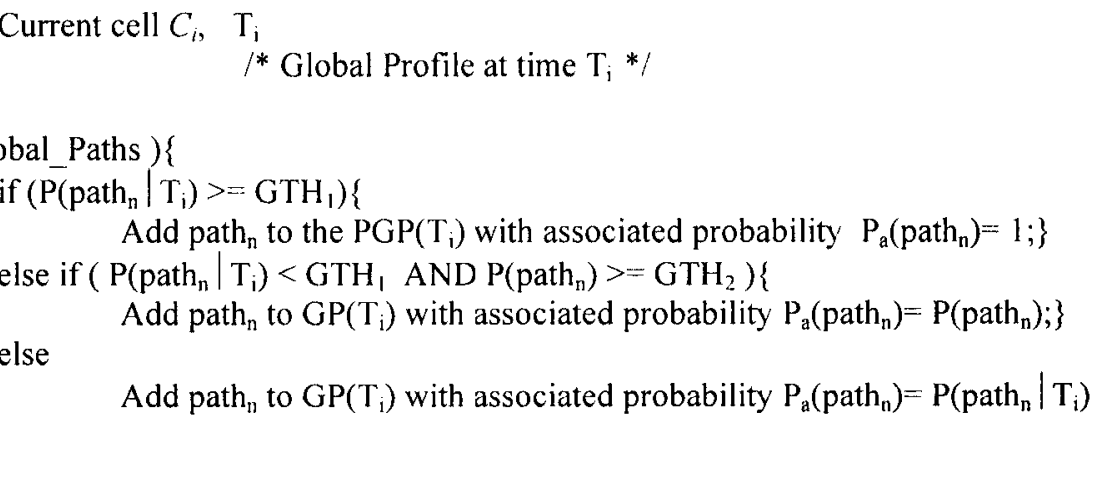 \\
\hline
\end{tabular}




\section{APPENDIX B}

B.1 Predictive resource reservation algorithm (RR) for PCAC-RR

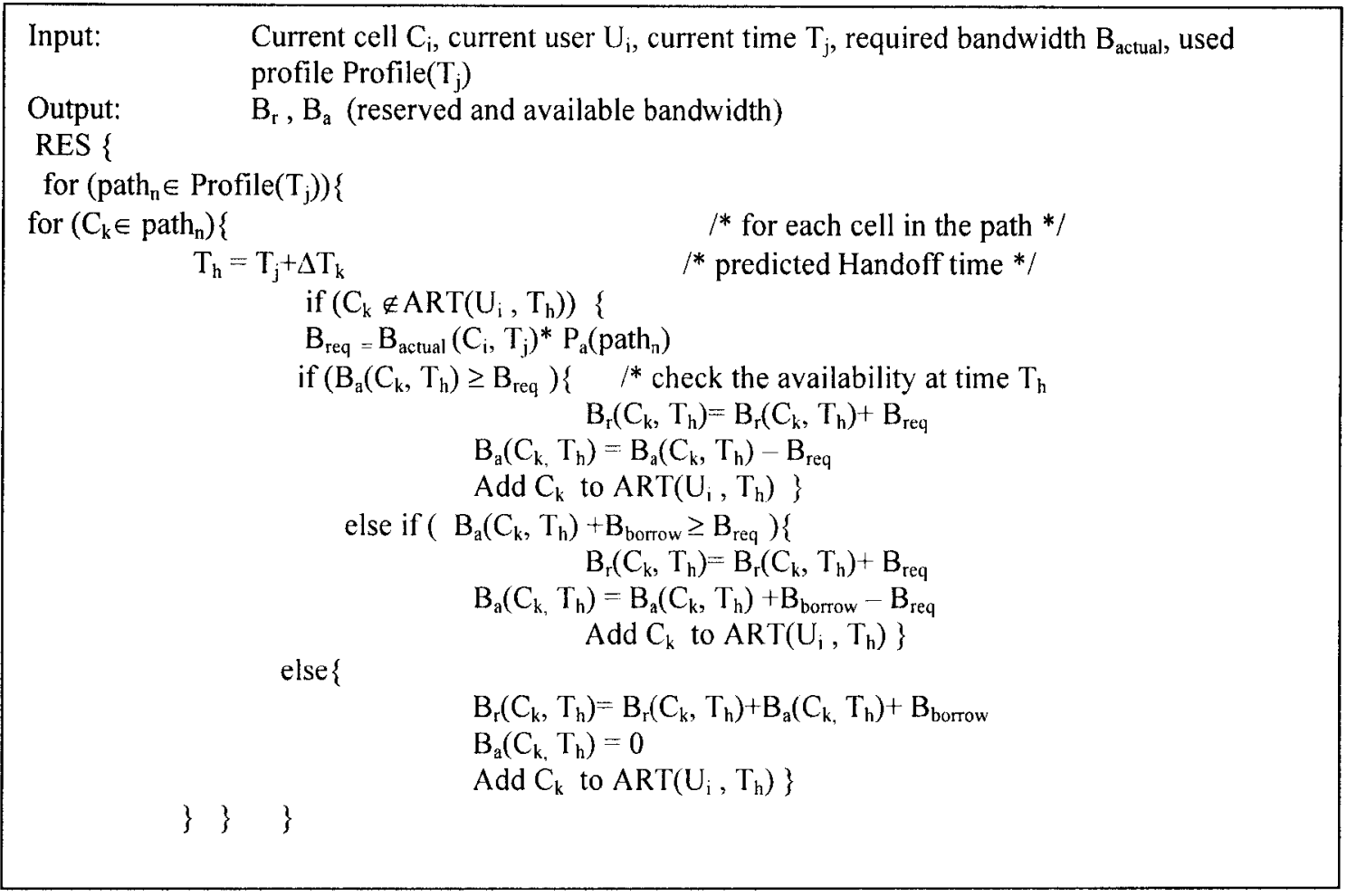




\section{B.2 Predictive CAC algorithm (PCAC) for PCAC-RR}

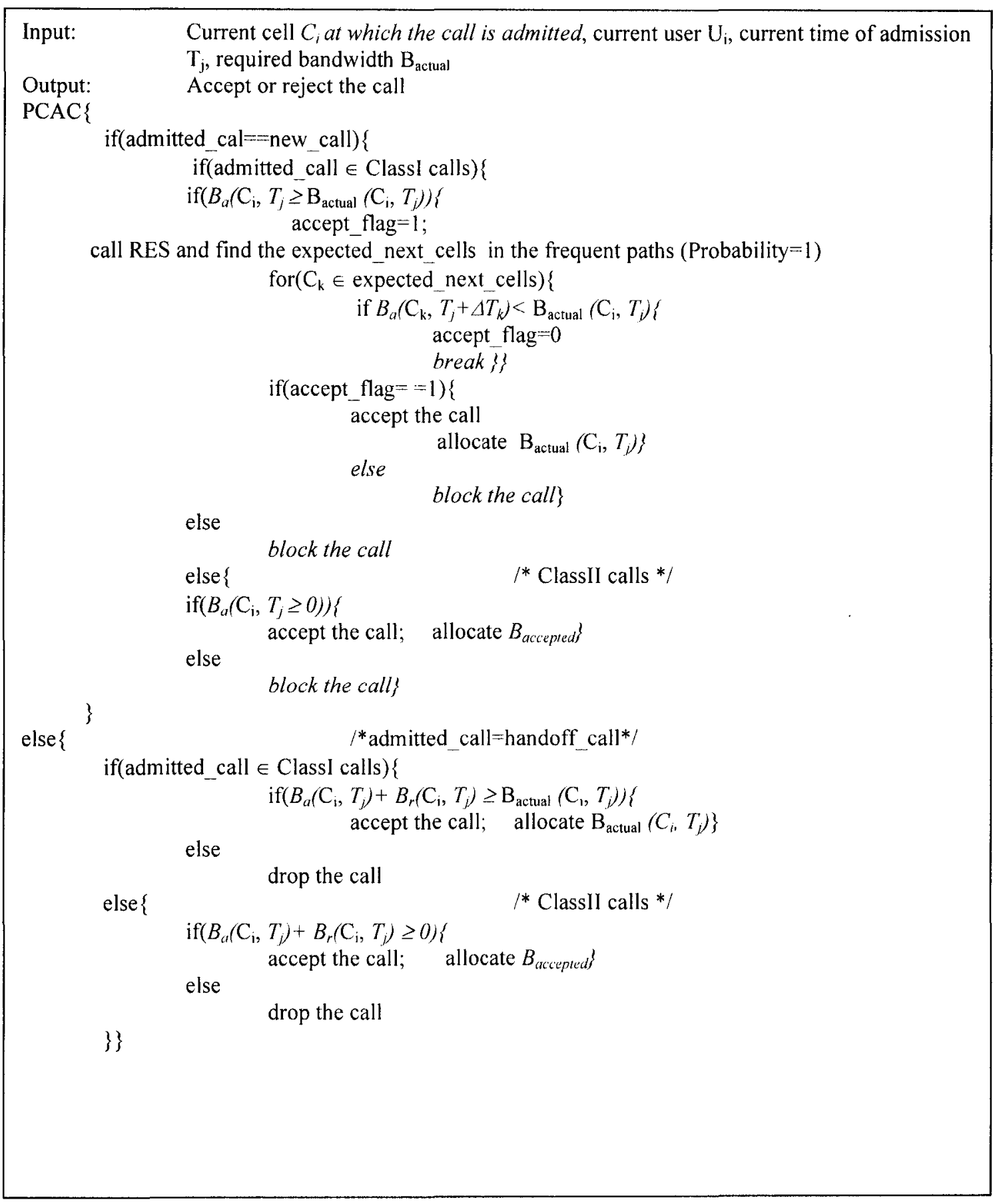




\section{APPENDIX C}

\section{MobilePrefixSpan Algorithm}

0. Prepare the multidimensional sequences and convert the problem to one-dimensional sequence mining.

1. Apply the first scan to find all of the single-item frequent sequences (prefix).

2. Find the projected dataset corresponding to the single-item frequent sequences.

3. We continue by finding out the single-item frequent sequences in each projected dataset.

4. Find the frequent sequences using this prefix:

- If the items represent BSs, then consider the consecutive order.

- Otherwise consider the order only.

5. Record the frequent sequences that have been found using this prefix.

6. Use each of these recorded frequent sequences as a prefix to find its projected dataset.

7. Repeat steps from 4 to 7 until we find all sequential patterns. 


\section{APPENDIX D}

\section{D1. Admission Control Algorithm for PCA-WHN}

\section{For New Calls:}

Case 1: If the class of the requested service(ReqServ) is better to be supported by another type of RAN:

1. Check the new RAN:

a. If there are resources available set the expected RAN AP/BS (in the $\mathrm{MH})$ to be the new RAN AP/BS.

b. If there are no resources available, the expected RAN AP/BS will be the current RAN AP/BS.

2. According to ReqServ, the Expected RAN AP/BS, the period of time and the current day, predict the future locations (BSs/APs) based on the MH mobility models.

3. If no frequent path could be predicted using sequential patterns stored in the $\mathrm{MH}$, then we have to access the sequential patterns stored in the BS/AP.

4. According to the predicted path, the load at each location, and the expected handoff time:

c. If the call is from CLASS I, check the available resources in all of the locations (BS/AP) in the expected path.

d. If the call is from CLASS II, check the available resources in next expected BS/AP (not all of the BS/AP on the path).

5. If there are available resources in the expected location(s):

e. Start a vertical handoff automatically to Expected RAN AP/BS if it is not equal to the current RAN AP/BS.

f. Accept the call and reserve the required resources in the future APs/BSs

6. Otherwise the call will be rejected. 
Case 2: If the class of the requested service(ReqServ) is better to be supported by the current RAN:

1. Check the current RAN availability:

a. If there are enough resources, set the Expected RAN AP/BS to the current RAN AP/BS.

b. If there no enough resources, set the Expected RAN AP/BS to the other RAN AP/BS.

2. According to ReqServ, the Expected RAN AP/BS, the period of time and the current day, find the future locations (BSs/APs) based on the MH mobility models.

3. If no frequent path could be predicted using sequential patterns stored in the $\mathrm{MH}$, then we have to access the sequential patterns stored in the BS/AP.

4. According to the expected path, the load at each location, the expected handoff time:

a. If the call is from CLASS I, check the available resources in all of the locations (BS/AP) in the expected path.

b. If the call is from CLASS II, check the available resources in next expected BS/AP (not all of the BS/AP on the path).

5. If there is available resources in the expected location(s):

c. Start a vertical handoff automatically to Expected RAN AP/BS if it is not equal to the current RAN AP/BS.

d. Accept the call and reserve the required resources in the future APs/BSs

6. Otherwise the call will be rejected.

For Handoff Calls:

1. If the handoff call is from CLASS I we can check the resources in the current BS/AP by checking the available resources and the reserved resources for both classes $\left(R_{a}+R_{h 2}+R_{h 1}\right)$ according to the time of service or the expected time of service.

2. If the handoff call is from CLASS II we can check the resources in the current BS/AP by checking the available resources and the reserved resources for CLASS Il $\left(\mathrm{R}_{\mathrm{a}}+\mathrm{R}_{\mathrm{h} 2}\right)$.

3. If there are resources available:
a. Accept the handoff call
b. Allocate the required resources
c. Update the current available resources

4. Otherwise drop the call. 


\section{D2. Benchmark technique for PCA-WHN}

For New Calls:

2. If it is better to support the required service by the current RAN, set the future locations (BSs/APs) to the (BSs/APs) in the current RAN that will cover his next movements (prediction is perfect) starting from the current (BSs/APs).

3. If it is better to support the required service by another RAN, set the future locations (BSs/APs) to the (BSs/APs) of the new RAN that will cover his actual locations in the future (prediction is perfect) .

4. According to the future locations, the load at each location, and the exact handoff time:

a. If the call is from CLASS I, check the available resources in all of the locations (BS/AP) in the future path.

b. If the call is from CLASS II, check the available resources in next BS/AP (not all of the BS/AP on the path).

5. If there are available resources in the future location(s):

a. If the new location will be in another RAN, start a vertical handoff automatically

b. Accept the call and reserve the required resources in the future APs/BSs

6. Otherwise the call will be rejected.

\section{For Handoff Calls:}

2. If the handoff call is from CLASS I we can check the resources in the current $\mathrm{BS} / \mathrm{AP}$ by checking the available resources and the reserved resources for both classes $\left(R_{a}+R_{h 2}+R_{h 1}\right)$ according to the exact time of service (prediction is perfect).

3. If the handoff call is from CLASS II we can check the resources in the current BS/AP by checking the available resources and the reserved resources for CLASS II $\left(\mathrm{R}_{\mathrm{a}}+\mathrm{R}_{\mathrm{h} 2}\right)$.

4. If there are resources available:

a. Accept the handoff call

b. Allocate the required resources

c. Update the current available resources

5. Otherwise drop the call. 


\section{APPENDIX E}

E1. Bandwidth Reservation Algorithm for PC-FCAT4 [28]

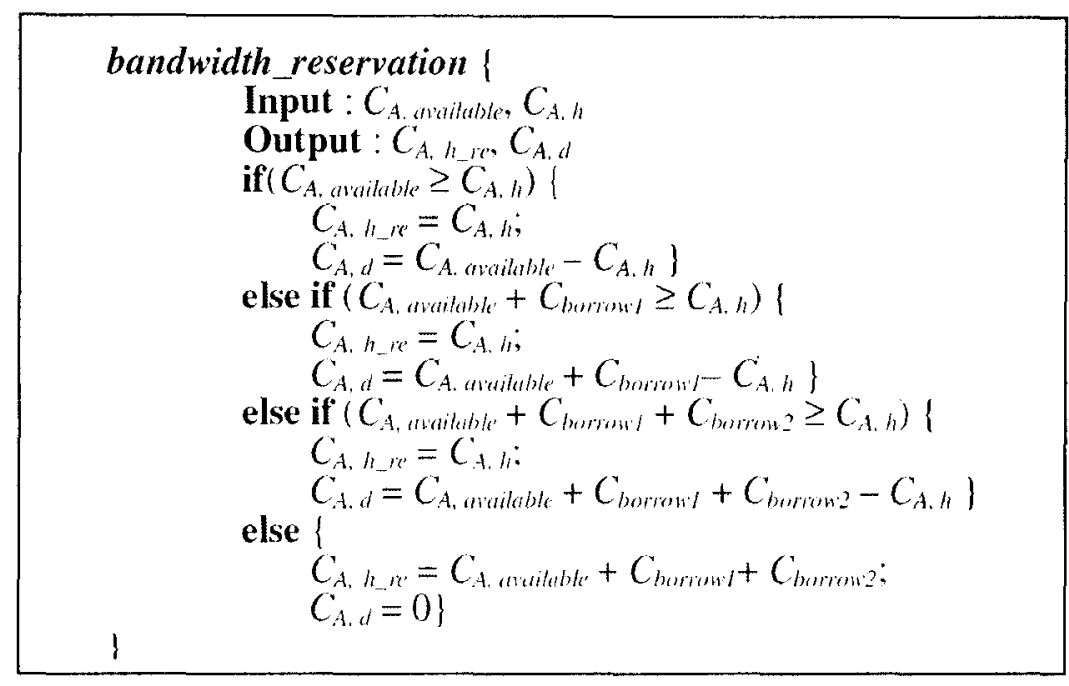

E2. Call Admission Control Algorithm for PC-FCAT4 [28]

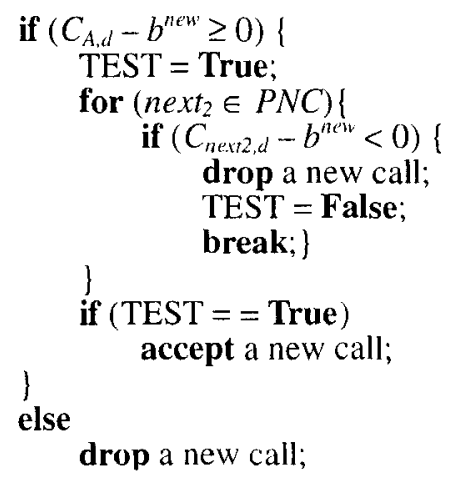




\section{CURRICULUM VITA}

- Name: $\quad$ Sherif Said Rashad

- Date of Birth: 28 September 1974, Cairo, Egypt

\section{- Education:}

- Ph.D., Computer Engineering and Computer Science Department, Speed School of Engineering, University of Louisville, Louisville, KY, USA, December 2006.

- M.Sc., Electronics and Communications Engineering Department, Faculty of Engineering, Zagazig University Zagazig, Egypt, September 2001.

- B.Sc. with Honors, Electronics and Communications Engineering Department, Faculty of Engineering, Zagazig University, Zagazig, Egypt, May 1996.

- Work Experience:

- University of Louisville, 2001-2006: Graduate research assistant / graduate teaching assistant / graduate fellow. Member of the Data Mining Lab and the Mobile Information Networks and Distributed Systems (MINDS) Lab

- Zagazig University, 1998-2001: Teaching and research assistant.

- Honors and Awards (During the Ph.D. Study)

- Computer Science and Engineering Doctoral Award, Speed School of Engineering, University of Louisville, 2006.

- First Recipient of Rebecca L. and Henry P. Conn Endowed Fellowship, Speed School of Engineering, University of Louisville, 2004- 2006. 
- Listed in Who's Who Among Students in American Universities and Colleges, 2006.

- Invited member, Upsilon Pi Epsilon, Computer Science Honor Society.

- Invited member, Golden Key International Honour Society.

- Certificate of recognition in the Engineers' Day from the Computer Engineering and Computer Science Department, University of Louisville, 2005 and 2004.

- Certificate of recognition for outstanding commitment and dedication as a member of the local organizing committee, IEEE and $A C M$ International Conference on Machine Learning and Applications (ICMLA), 2004.

\section{- Publications from this Ph.D. Dissertation}

\section{Journal Papers and Book Chapters}

1. Sherif Rashad, Mehmed Kantardzic, and Anup Kumar, "User Mobility Oriented Predictive Call Admission Control and Resource Reservation for Next-Generation Mobile Networks", Journal of Parallel and Distributed Computing (JPDC), Elsevier, Volume 66, Issue 7, pp. 971-988, July 2006.

2. Sherif Rashad, Mehmed Kantardzic, and Anup Kumar, "Time Component Analysis of Enhanced Predictive Call Admission Control in Wireless Cellular Networks", GESTS Int'l Transaction on Computer Science and Engineering, Vol.7, No.1, pp.75-86, May 2005.

3. Sherif Rashad, Mehmed Kantardzic, and Anup Kumar, "A Data Mining Approach for Call Admission Control and Resource Reservation in Wireless Mobile Networks", book chapter in "Advances in Data Mining Applications in Image Mining, Medicine and Biotechnology, Management and Environmental Control, and Telecommunications", Petra Perner (Editor), Springer Verlag, LNCS: LNAI Vol. 3275, pp. 134-143, 2004.

4. Sherif Rashad, Mehmed Kantardzic, and Anup Kumar, "PAC-WHN: Predictive Admission Control for Wireless Heterogeneous Networks", in submission.

\section{Conference Papers}

5. Sherif Rashad, Mehmed Kantardzic, and Anup Kumar, "MSP-CACRR: Multidimensional Sequential Patterns Based Call Admission Control and Resource Reservation for Next-Generation Wireless Cellular Networks", in review, Nov. 2006. 
6. Sherif Rashad, Mehmed Kantardzic, and Anup Kumar, "Mobile Data Mining for Radio Resource Management in Wireless Mobile Networks", Proceedings of the 2004 IEEE and ACM International Conference on Machine Learning and Applications (ICMLA 2004), pp.85-92, Louisville, Kentucky, 16-18 December 2004.

7. Sherif Rashad, Mehmed Kantardzic, and Anup Kumar, "PCAC-RR: A Predictive Call Admission Control and Resource Reservation for Wireless Mobile Networks", Proceedings of the IEEE and IFIP International Conference on Wireless and Optical Communications Networks (WOCN 2004), pp. 295-298, Muscat, Oman, 7-9 June 2004.

8. Sherif Rashad, Mehmed Kantardzic, Anup Kumar, "A Data Mining Approach for Call Admission Control and Resource Reservation in Wireless Mobile Networks", Proceedings of 4th Industrial Conference on Data Mining (ICDM'2004), Leipzig, Germany, 4-7 July 2004 (a revised extended version was selected for publication as a book chapter in LNCS).

\section{- Professional Memberships}

- Institute of Electrical and Electronics Engineers (IEEE).

- IEEE Computer Society and IEEE Communication Society.

- IEEE Technical Committee on Computer Communications (TCCC).

- IEEE Technical Committee on Personal Communications (TCPC).

- Association for Computing Machinery (ACM).

- International Engineering Consortium

- Egyptian Syndicate of Engineers.

\section{- Reviewer and Technical Program Committee Member for:}

- IEEE International Conference on Communications (ICC 2007), Multimedia Communications and Home Services Symposium (MCH).

- International Resource Management Association Conference (IRMA 2007).

- Services

- Participated in organizing the 5th IEEE Symposium on Signal Processing and Information Technology (ISSPIT'05), Athens, Greece, December 18-21, 2005. 
- Participated in organizing the 2004 IEEE and ACM International Conference on Machine Learning and Applications (ICMLA'04), Louisville, KY, December 16-18, 2004.

- Participated in organizing the 9th IEEE International Symposium on Computers and Communications (ISCC'04). Alexandria, Egypt, June 28 - July 1, 2004.

- Participated in organizing the 4th IEEE Symposium on Signal Processing and Information Technology (ISSPIT'04), Rome, Italy, December 18-21, 2004.

- Was elected as the Vice-President of the Egyptian Students Association in North America (ESANA), Louisville Unit, KY, May 2003 - April 2004. 